\title{
AFFINE COHOMOLOGICAL TRANSFORMS, PERVERSITY, AND MONODROMY
}

\author{
NICHOLAS M. KATZ
}

TABLE OF CONTENTS

Introduction

I. The main technical result

II. Results on irreducibility and purity

III. Applications to Bertini and Lefschetz-style theorems for hypersurface sections

IV. Diophantine applications

V. Calculation of some monodromy groups

Appendix (d'apres Deligne)

References

\section{INTRODUCTION}

It is now nearly a decade since the theories of perverse sheaves and of the $l$ adic Fourier transform came into being and gave fundamental new insights into the behavior of additive character sums over finite fields. Most of these insights were spellings out of the basic fact that the Fourier transform of a perverse sheaf is itself a perverse sheaf, a statement that amounts to a succession of vanishing statements for various compactly supported cohomology groups. This vanishing, applied to input perverse sheaves that are "mixed", is transformed by Deligne's fundamental Weil II results into archimedean estimates for the corresponding sums.

In this paper, we show that a wide class of $l$-adic "affine cohomological transforms" shares with the Fourier transform the fundamental property of carrying perverse sheaves to perverse sheaves.

To define these transforms, fix a field $k$ of finite characteristic $p>0$, a prime number $l \neq p$, integers $n \geq 1$ and $m \geq 1$, an affine $k$-scheme $V$ of

Received by the editors October 7, 1991.

1991 Mathematics Subject Classification. Primary 14F20, 14F32, 14G15, 32S40. 
finite type, a $k$-morphism $f: V \rightarrow \mathbb{A}^{m}$, a quasi-finite $k$-morphism $\pi: V \rightarrow \mathbb{A}^{n}$, and a "kernel" $K$ in $D_{c}^{b}\left(\mathbb{A}^{m}, \bar{Q}_{l}\right)$. We denote by $\operatorname{Aff} \operatorname{Maps}\left(\mathbb{A}^{n}, \mathbb{A}^{m}\right)$ the affine space of affine maps $x \mapsto A x+b$ from $\mathbb{A}^{n}$ to $\mathbb{A}^{m}$. We consider the morphism

$$
f_{\text {aff }}: V \times \operatorname{Aff} \operatorname{Maps}\left(\mathbb{A}^{n}, \mathbb{A}^{m}\right) \rightarrow \mathbb{A}^{m}
$$

defined by

$$
f_{\mathrm{aff}}:(v, A, b) \mapsto f(v)+A \pi(v)+b:=f_{A, b}(v) .
$$

We get a transform from $D_{c}^{b}\left(V, \overline{\mathbb{Q}}_{l}\right)$ to $D_{c}^{b}\left(\operatorname{Aff} \operatorname{Maps}\left(\mathbb{A}^{n}, \mathbb{A}^{m}\right), \overline{\mathbb{Q}}_{l}\right)$ by mapping $L$ on $V$ to $R\left(p r_{2}\right)_{!}\left(p r_{1}^{*} L \otimes f_{\text {aff }}^{*} K\right)[n m]$ on $\operatorname{Aff} \operatorname{Maps}\left(\mathbb{A}^{n}, \mathbb{A}^{m}\right)$ via the correspondence diagram

$$
\begin{aligned}
& L \text { on }{ }_{p r_{1}} \quad K \text { on } \\
& V \stackrel{p r_{1}}{\longleftarrow} V \times \operatorname{Aff} \operatorname{Maps}\left(\mathbb{A}^{n}, \mathbb{A}^{m}\right) \stackrel{f_{\text {aff }}}{\longrightarrow} \quad \mathbb{A}^{m} \\
& \downarrow p r_{2} \\
& \operatorname{Aff} \operatorname{Maps}\left(\mathbb{A}^{n}, \mathbb{A}^{m}\right)
\end{aligned}
$$

The main technical result is that, if the kernel $K$ is a perverse sheaf on $\mathbb{A}^{m}$ with the property that $H_{c}\left(\mathbb{A}^{m} \otimes \bar{k}, K\right)=0$, then the transform

$$
L \mapsto R\left(p r_{2}\right)_{!}\left(p r_{1}^{*} L \otimes f_{\text {aff }}^{*} K\right)[n m]
$$

carries perverse sheaves on $V$ to perverse sheaves on Aff Maps. This is the main content of $\S 1$.

When the field $k$ is finite, the diophantine interpretation of such transforms is given by the Lefschetz Trace Formula. For any finite extension $E$ of $k$, the trace function of the transform of $L$ on $E$-valued points of Aff Maps is given in terms of the trace functions $K(x, E)$ and $L(v, E)$ of $K$ and $L$ on $E$-valued points of $\mathbb{A}^{m}$ and $V$ respectively by the rule

$$
(A, b) \text { in } \operatorname{Aff} \operatorname{Maps}(E) \mapsto(-1)^{n m} \sum_{V(E)} L(v, E) K(f(v)+A \pi(v)+b, E) .
$$

To the extent that the trace functions of $K$ and $L$ have interpretations as some sort of "exponential sums", the trace function of the transform is thus a "sum of exponential sums".

Where does the original Fourier transform fit into this picture? Take $m=1$ and $K$ the perverse sheaf $\mathscr{L}_{\psi}[1]$ on $\mathbb{A}^{1}$, where $\mathscr{L}_{\psi}$ is the lisse Artin-Schreier sheaf of rank one on $\mathbb{A}^{1}$ corresponding to a nontrivial additive character $\psi$ of a finite subfield of $k$. The resulting transform of $L$ is essentially the Fourier transform of $\pi_{i}\left(L \otimes \mathscr{L}_{\psi(f)}\right)$.

[To be more precise, if we view $\operatorname{Aff} \operatorname{Maps}\left(\mathbb{A}^{n}, \mathbb{A}^{1}\right)$ as the product of Lin Maps $\left(\mathbb{A}^{n}, \mathbb{A}^{1}\right)$ with $\mathbb{A}^{1}$, corresponding to coordinates $(A, b)$, then the transform of $L$ is the external tensor product of the Fourier transform $\mathrm{FT}_{\psi}\left(\pi_{!}\left(L \otimes \mathscr{L}_{\psi(f)}\right)\right)$ on $\operatorname{Lin} \operatorname{Maps}\left(\mathbb{A}^{n}, \mathbb{A}^{1}\right)$ with $\mathscr{L}_{\psi}[1]$ on $\mathbb{A}^{1}$.]

But there are a plethora of perverse sheaves $K$ on $\mathbb{A}^{1}$ with the property that $H_{c}\left(\mathbb{A}^{1} \otimes \bar{k}, K\right)=0$, and each of them defines an affine cohomological transform, which preserves perversity. 
Perhaps the most striking examples, after $\mathscr{L}_{\psi}[1]$, of perverse sheaves $K$ on $\mathbb{A}^{1}$ with the property that $H_{c}\left(\mathbb{A}^{1} \otimes \bar{k}, K\right)=0$ are the perverse sheaves $j_{!} \mathscr{L}_{\chi}[1]$, where $\mathscr{L}_{\chi}$ is the lisse rank one Kummer sheaf on $\mathbb{G}_{m}$ defined by a nontrivial multiplicative character $\chi$ of a finite subfield of $k$, and $j: \mathbb{G}_{m} \rightarrow \mathbb{A}^{1}$ is the inclusion.

That the theory applies both to $\mathscr{L}_{\psi}[1]$ and by $j_{1} \mathscr{L}_{\chi}[1]$ may be regarded as a "weak unification" of the theory of additive and of multiplicative character sums. To fix ideas, suppose that $k$ is a finite field, and that $V$ is smooth and geometrically irreducible of some dimension $d$, so that the shifted constant sheaf $\overline{\mathbb{Q}}_{l, V}[d]$ is perverse on $V$ and, hence, may serve as an $L$. Let us recall the crude diophantine consequences of the fact that the transforms defined by $\mathscr{L}_{\psi}[1]$ and by $j_{1} \mathscr{L}_{\chi}[1]$ preserve perversity.

With $K=\mathscr{L}_{\psi}[1]$ and $L=\overline{\mathbb{Q}}_{l, V}[d]$, the trace function of

$$
R\left(p r_{2}\right)_{!}\left(p r_{1}^{*} L \otimes f_{\mathrm{aff}}^{*} K\right)[n]
$$

on $E$-valued points, where $E$ is any finite extension of $k$, is the additive character sum

$$
(-1)^{n+d+1} \sum_{V(E)} \psi_{E}(f(v)+A \pi(v)+b),
$$

where $\psi_{E}$ is the composition of $\psi$ with the trace from $E$ to $k$.

With $K=j_{!} \mathscr{L}_{\chi}[1]$ and $L=\overline{\mathbb{Q}}_{l, V}[d]$, the trace function of

$$
R\left(p r_{2}\right)_{!}\left(p r_{1}^{*} L \otimes f_{\mathrm{aff}}^{*} K\right)[n]
$$

on $E$-valued points, where $E$ is any finite extension of $k$, is the multiplicative character sum

$$
(-1)^{n+d+1} \sum_{V(E)} \chi_{E}(f(v)+A \pi(v)+b),
$$

where $\chi_{E}$ on $E^{\times}$is the composition of $\chi$ with the norm from $E^{\times}$to $k^{\times}$, extended by zero to all of $E$.

In each of these cases, the perversity, together with Deligne's Weil II, implies that for each given nontrivial $\psi$ or $\chi$, say $\rho$, there exists a constant $C_{\rho}$ and a dense open set $U_{\rho}$ of $(A, b)$ 's over which we have the estimate

$$
\left|\sum_{V(E)} \rho_{E}(f(v)+A \pi(v)+b)\right| \leq C_{\rho}\left(q_{E}\right)^{d / 2} .
$$

The diophantine consequences of the general theory are worked out in $\S I V$.

Other perverse sheaves $K$ on $\mathbb{A}^{1}$ with the property that $H_{c}\left(\mathbb{A}^{1} \otimes \bar{k}, K\right)=0$ are the sheaves $j_{*} \mathscr{F}[1], j: \mathbb{G}_{m} \rightarrow \mathbb{A}^{1}$ the inclusion, where $\mathscr{F}$ is either a Kloosterman sheaf $\mathrm{Kl}_{\psi}\left(\chi_{1}, \ldots, \chi_{n}\right)$ or more generally a hypergeometric sheaf $\mathscr{H}_{\lambda}\left(!, \psi, \chi_{1}, \ldots, \chi_{n} ; \rho_{1}, \ldots, \rho_{m}\right)$, at least one of whose $\chi_{i}$ is trivial.

Still with $m=1$, yet another perverse sheaf $K$ on $\mathbb{A}^{1}$ with the property that $H_{c}\left(\mathbb{A}^{1} \otimes \bar{k}, K\right)=0$ is $R j_{*} \overline{\mathbb{Q}}_{l}[1]$, for $j: \mathbb{G}_{m} \rightarrow \mathbb{A}^{1}$ the inclusion. The fact that the corresponding transform preserves perversity implies a quite general form 
of the weak Lefschetz theorem; this is the subject of $\S$ III. Deligne has explained to me a simpler, more direct proof of weak Lefschetz. His proof is given in the Appendix.

In addition to preserving perversity, another special property of the Fourier transform is to carry perverse irreducibles to perverse irreducibles. Our more general transforms lack this property in general; however, if $m=1$ and if the map $\pi: V \rightarrow \mathbb{A}^{n}$ is a closed immersion, then for a given perverse irreducible $L$ on $V$, its transform $R\left(p r_{2}\right)_{!}\left(p r_{1}^{*} L \otimes f_{\text {aff }}^{*} K\right)[n]$ will be perverse irreducible on Aff Maps provided we take $K$ to $j_{!} \mathscr{L}_{\chi}[1]$ with $\chi$ a multiplicative character of sufficiently high order. By the Fourier transform theory, we also have this irreducibility if we take $K$ to be $\mathscr{L}_{\psi}[1]$. In this sense, too, our theory "unifies" results for nontrivial additive $\psi$ 's and for multiplicative $\chi$ 's of sufficiently high order.

In the case $m \geq 1$ and the map $\pi: V \rightarrow \mathbb{A}^{n}$ a closed immersion, we prove irreducibility results of a similar flavor. These include a result conjectured by Dwork and the $l$-adic analogue of a $\mathscr{D}$-module result of [GKZ] over $\mathbb{C}$. We also prove under these conditions that over a finite field, if $K$ and $L$ are each perverse irreducible and pure, then the "highest weight part" of the transform $R\left(p r_{2}\right)_{1}\left(p r_{1}^{*} L \otimes f_{\text {aff }}^{*} K\right)[n m]$ is perverse irreducible. These irreducibility results are worked out in $\S I I$.

In $\S \mathrm{V}$, we compute the monodromy groups attached to (the restriction to a dense open set of $\operatorname{Aff} \operatorname{Maps}\left(\mathbb{A}^{1}, \mathbb{A}^{1}\right)$, where it is the shift of a single lisse sheaf) the transforms corresponding to the two-parameter family of multiplicative character sums in one variable

$$
(a, b) \mapsto \sum_{\mathbb{A}^{1}} \chi(f(x)+a x+b)
$$

(With $\chi$ replaced by $\psi$, the monodromy was computed in [Ka3].) For $n>1$ and a polynomial $f$ in $n$ variables, it would be very interesting to compute the monodromy groups of the $(n+1)$-parameter families of character sums in $n$ variables

$$
\left(a_{1}, \ldots, a_{n}, b\right) \mapsto \sum_{\mathbb{A}^{n}} \rho\left(f(x)+\sum a_{i} x_{i}+b\right),
$$

both for $\rho=\psi$ and for $\rho=\chi$.

I first learned the idea that character sums involving a polynomial $f$ in $n$ variables "get better" if one adds to $f$ a general $\sum a_{i} x_{i}+b$ term and that one can say interesting things about the dependence of these sums on $\left(a_{i}\right.$ 's, $\left.b\right)$, from a paper [DL] of Davenport-Lewis, cf. also [Ka2]. The idea of thinking systematically about "sums of exponential sums", e.g., taking a kernel $K$ corresponding to Kloosterman sums, was inspired by a question Iwaniec once asked me about certain sums of Kloosterman sums. I would like to thank Steve Sperber for the use of his notes of lectures I gave at the University of Minnesota in July 1990 on the material in $\S \S I-I V$ of this paper.

\section{THE MAIN TECHNICAL RESULT}

1.0. Throughout this section, we work over a field $k$ of characteristic $p$. We fix a prime number $l \neq p$. On variable separated $k$-schemes of finite type $X$ 
we will work systematically with objects of $D_{c}^{b}\left(X, \overline{\mathbb{Q}}_{l}\right)$.

1.1. Recall [BBD, Chapter 4] that an object $K$ of $D_{c}^{b}\left(X, \overline{\mathbb{Q}}_{l}\right)$ is called semiperverse if its cohomology sheaves $\mathscr{H}^{i}(K)$ satisfy

$$
\operatorname{dim} \operatorname{Supp}\left(\mathscr{H}^{i}(K)\right) \leq-i, \quad \text { for every integer } i \text {. }
$$

An object $K$ is called perverse if both $K$ And its Verdier dual

$$
D_{X / k} K:=\operatorname{RHom}\left(K, f^{!} \overline{\mathbb{Q}}_{l}\right),
$$

$f: X \rightarrow \operatorname{Spec}(k)$ denoting the structural morphism, are semiperverse. The main facts about perversity, semiperversity, and duality that we will use are the following [BBD, Chapter 4].

(1.1.1) If $f: X \rightarrow Y$ is an affine morphism, then $K \mapsto R f_{*} K$ preserves semiperversity.

(1.1.2) If $f: X \rightarrow Y$ is a quasi-finite morphism, then $K \mapsto R f_{!} K$ preserves semiperversity.

(1.1.3) If $f: X \rightarrow Y$ is an arbitrary morphism whose geometric fibres all have dimension $\leq d$, then $L \mapsto f^{*} L[d]$ preserves semiperversity.

(1.1.4) Duality interchanges $R f_{!}$and $R f_{*}$.

(1.1.5) Duality interchanges $f^{!}$and $f^{*}$.

(1.1.6) If $f: X \rightarrow Y$ is a smooth morphism everywhere of relative dimension $d$, then $f^{!}=f^{*}[2 d](d)$. Consequently $f^{*}[d](d / 2)$ is self-dual and $K \mapsto f^{*} K[d]$ preserves perversity.

(1.1.7) If $X$ is smooth over $k$, purely of dimension $d$, then for any lisse $\overline{\mathbb{Q}}_{l}$-sheaf $\mathscr{F}$ on $X, \mathscr{F}[d]$ is preverse and $D_{X / k}(\mathscr{F}[d])=\mathscr{F}^{\vee}[d](d)$.

(1.1.8) If $X$ is a local complete intersection over $k$, purely of dimension $d$, then for any lisse $\overline{\mathbb{Q}}_{l}$-sheaf $\mathscr{F}$ on $X, \mathscr{F}[d]$ is preverse.

1.2. For each integer $r \geq 0$, we denote by $\mathbb{A}_{k}^{r}$, or just $\mathbb{A}^{r}$ if no confusion is likely, the $r$-dimensional affine space over $k$.

Fix nonnegative integers $n, m$, and consider the following data:

$V$, a separated $k$-scheme of finite type;

$f: V \rightarrow \mathbb{A}^{m}$ an arbitrary morphism;

$\pi: V \rightarrow \mathbb{A}^{n}$ a quasi-finite morphism;

$K$, semiperverse on $\mathbb{A}^{m}$;

$L$, semiperverse on $V$.

1.3. We denote by $\operatorname{Aff} \operatorname{Maps}\left(\mathbb{A}^{n}, \mathbb{A}^{m}\right)$ the affine space over $k$ of dimension $n m+m$ consisting of all affine maps from $\mathbb{A}^{n}$ to $\mathbb{A}^{m}$, i.e., the space of all maps from $\mathbb{A}^{n}$ to $\mathbb{A}^{m}$ of the form $x \mapsto A x+b$, with $A$ a linear map from $\mathbb{A}^{n}$ to $\mathbb{A}^{m}$ and with $b$ in $\mathbb{A}^{m}$. We will systematically use the coordinates $(A, b)$ on Aff to identify it with the product

$$
\operatorname{Aff} \operatorname{Maps}\left(\mathbb{A}^{n}, \mathbb{A}^{m}\right)=\operatorname{Lin} \operatorname{Maps}\left(\mathbb{A}^{n}, \mathbb{A}^{m}\right) \times \mathbb{A}^{m} \text {. }
$$

1.4. We now consider the morphism

$$
\begin{aligned}
& f_{\mathrm{aff}}: V \times \operatorname{Aff} \operatorname{Maps}\left(\mathbb{A}^{n}, \mathbb{A}^{m}\right) \rightarrow \mathbb{A}^{m}, \\
& f_{\mathrm{aff}}:(v, A, b) \mapsto f(v)+A \pi(v)+b:=f_{A, b}(v)
\end{aligned}
$$


and the correspondence diagram

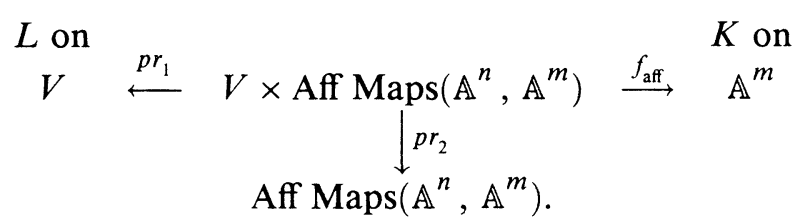

1.5. Main Theorem. Notation and hypotheses are as above, i.e.,

$k$, a field of characteristic $p \neq l$;

$V$, a separated $k$-scheme of finite type;

$f: V \rightarrow \mathbb{A}^{m}$, an arbitrary morphism;

$\pi: V \rightarrow \mathbb{A}^{n}$, a quasi-finite morphism;

$K$, semiperverse on $\mathbb{A}^{m}$;

$L$, semiperverse on $V$.

Suppose further that either $H_{c}\left(\mathbb{A}^{m} \otimes \bar{k}, K\right)=0$ or $H_{c}(V \otimes \bar{k}, L)=0$. Then the object $M:=R\left(p r_{2}\right)_{!}\left(p r_{1}^{*} L \otimes f_{\text {aff }}^{*} K\right)[n m]$ on Aff $\operatorname{Maps}\left(\mathbb{A}^{n}, \mathbb{A}^{m}\right)$ is semiperverse, and

$$
H_{c}\left(\operatorname{Aff} \operatorname{Maps}\left(\mathbb{A}^{n}, \mathbb{A}^{m}\right) \otimes \bar{k}, M\right)=0 .
$$

Before beginning the proof, we give the main application.

1.6. Perversity Corollary. Hypotheses and notation are as in Theorem 1.5. We have:

(1) The dual $D M$ of $M$ is $R\left(p r_{2}\right)_{*}\left(p r_{1}^{*} D L \otimes f_{\mathrm{aff}}^{*} D K\right)[n m](n m)$.

(2) Suppose in addition that

$V$ is affine,

$K$ is preverse on $\mathbb{A}^{m}$,

$L$ is perverse on $V$.

Then $M$ is perverse on $\operatorname{Aff} \operatorname{Maps}\left(\mathbb{A}^{n}, \mathbb{A}^{m}\right)$.

Proof. (1) Because duality interchanges $R\left(p r_{2}\right)_{\text {! }}$ and $R\left(p r_{2}\right)_{*}$, we have

$$
D M=R\left(p r_{2}\right)_{*} D\left(\left(p r_{1}^{*} L \otimes f_{\mathrm{aff}}^{*} K\right)[n m]\right) .
$$

In the coordinates $(v, A, b)$ on $V \times \operatorname{Aff} \operatorname{Maps}\left(\mathbb{A}^{n}, \mathbb{A}^{m}\right)$, which identify

$$
V \times \operatorname{Aff} \operatorname{Maps}\left(\mathbb{A}^{n}, \mathbb{A}^{m}\right)=V \times \operatorname{Lin} \operatorname{Maps}\left(\mathbb{A}^{n}, \mathbb{A}^{m}\right) \times \mathbb{A}^{m},
$$

consider the automorphism $\sigma$ defined by

$$
\sigma:(v, A, b) \mapsto(v, A, b+A \pi(v)+f(v)) .
$$

In terms of the projections of $V \times \operatorname{Lin} \operatorname{Maps}\left(\mathbb{A}^{n}, \mathbb{A}^{m}\right) \times \mathbb{A}^{m}$ onto its three factors, we have

$$
p r_{1}=p r_{1} \circ \sigma, \quad p r_{2}=p r_{2} \circ \sigma, \quad f_{\mathrm{aff}}=p r_{3} \circ \sigma
$$

Therefore

$$
\begin{aligned}
\left(p r_{1}^{*} L \otimes f_{\mathrm{aff}}^{*} K\right)[n m] & =\left(p r_{1}^{*} L\right) \otimes\left(p r_{2}^{*} \overline{\mathbb{Q}}_{l, \text { Lin Maps }}[n m]\right) \otimes\left(f_{\mathrm{aff}}^{*} K\right) \\
& =\sigma^{*}\left(\left(p r_{1}^{*} L\right) \otimes\left(p r_{2}^{*} \overline{\mathbb{Q}}_{l, \text { Lin Maps }}[n m]\right) \otimes\left(p r_{3}^{*} K\right)\right)
\end{aligned}
$$


Now $D \circ \sigma^{*}=\sigma^{!} \circ D=\sigma^{*} \circ D$, since $\sigma^{!}=\sigma^{*}$ for an automorphism $\sigma$, so we have, in fact,

$$
\begin{aligned}
D\left(\left(p r_{1}^{*} L \otimes f_{\mathrm{aff}}^{*} K\right)[n m]\right) \\
\quad=D\left(\sigma^{*}\left(\left(p r_{1}^{*} L\right) \otimes\left(p r_{2}^{*} \overline{\mathbb{Q}}_{l, \text { Lin Maps }}[n m]\right) \otimes\left(p r_{3}^{*} K\right)\right)\right) \\
\quad=\sigma^{*} D\left(\left(p r_{1}^{*} L\right) \otimes\left(p r_{2}^{*} \overline{\mathbb{Q}}_{l, \text { Lin Maps }}[n m]\right) \otimes\left(p r_{3}^{*} K\right)\right) \\
\quad=\sigma^{*}\left(\left(p r_{1}^{*} D L\right) \otimes\left(p r_{2}^{*} \overline{\mathbb{Q}}_{l, \text { Lin Maps }}[n m](n m)\right) \otimes\left(p r_{3}^{*} D K\right)\right) \\
\quad=\left(p r_{1}^{*} D L \otimes f_{\mathrm{aff}}^{*} D K\right)[n m](n m) .
\end{aligned}
$$

This proves (1).

For (2) we argue as follows. In view of Theorem 1.5, $M$ is semiperverse, so we need show only that its dual $D M$ is semiperverse. Because $V$ is affine, the morphism $p r_{2}$ is affine, so it suffices to show that $D\left(\left(p r_{1}^{*} L \otimes f_{\text {aff }}^{*} K\right)[n m]\right)$ is semiperverse. We will show that it is in fact perverse. In the proof of (1), we saw that

$$
D\left(\left(p r_{1}^{*} L \otimes f_{\mathrm{aff}}^{*} K\right)[n m]\right)=\sigma^{*}\left(\left(p r_{1}^{*} D L\right) \otimes\left(p r_{2}^{*} \overline{\mathbb{Q}}_{l, \text { Lin Maps }}[n m](n m)\right) \otimes\left(p r_{3}^{*} D K\right)\right)
$$

is the pullback by an automorphism of the external tensor product of perverse sheaves $D L, \overline{\mathbb{Q}}_{l, \text { Lin Maps }}[\mathrm{nm}]$, and $D K$ on the three factors and so is perverse [BBD, 4.2.8], as required. Q.E.D.

1.6bis. (Dual) Perversity Corollary. Notation and hypotheses are as above, i.e.,

$k$, a field of characteristic $p \neq l$;

$V$, a separated $k$-scheme of finite type;

$f: V \rightarrow \mathbb{A}^{m}$, an arbitrary morphism;

$\pi: V \rightarrow \mathbb{A}^{n}$, a quasi-finite morphism;

$K$, perverse on $\mathbb{A}^{m}$;

$L$, perverse on $V$.

Suppose further that $V$ is affine and that either $H\left(\mathbb{A}^{m} \otimes \bar{k}, K\right)=0$ or $H(V \otimes \bar{k}, L)=0$. Then the object $M:=R\left(p r_{2}\right)_{*}\left(p r_{1}^{*} L \otimes f_{\text {aff }}^{*} K\right)[n m](n m)$ on $\operatorname{Aff} \operatorname{Map}\left(\mathbb{A}^{n}, \mathbb{A}^{m}\right)$ is perverse, and

$$
H\left(\operatorname{Aff} \operatorname{Maps}\left(\mathbb{A}^{n}, \mathbb{A}^{m}\right) \otimes \bar{k}, M\right)=0 .
$$

Proof. This is the previous result, with $K, L$, and $M$ replaced by their duals $D K, D L$, and $D M$. Q.E.D.

\subsection{Proof of the main theorem, via Fourier Transform.}

1.7.1. Our main technical tool will be the Fourier Transform. Fix a nontrivial additive $\bar{Q}_{l}$-valued character $\psi$ of $\mathbb{F}_{p}$, so that we may speak of the sheaf $\mathscr{L}_{\psi}$ on $\mathbb{A}_{\mathbb{F}_{p}}^{1}$. For any $\mathbb{F}_{p}$-scheme $X$ and any function $f$ on $X$, viewed as a morphism to $\mathbb{A}_{\mathbb{F}_{p}}^{1}$, we may form $\mathscr{L}_{\psi(f)}:=f^{*} \mathscr{L}_{\psi}$ on $X$. For $S$ an arbitrary $\mathbb{F}_{p}$-scheme, $E$ a vector bundle over $S$ of some rank $r$, and $E^{\vee}$ the dual vector bundle, with duality pairing $\left(e, e^{\vee}\right)$, the Fourier Transform $\mathrm{FT}_{\psi}$ from $D_{c}^{b}\left(E, \overline{\mathbb{Q}}_{l}\right)$ to $D_{c}^{b}\left(E^{\vee}, \overline{\mathbb{Q}}_{l}\right)$ is defined in terms of the two projections of $E \times E^{\vee}$ 
onto its factors by

$$
\mathrm{FT}_{\psi}(K):=R\left(p r_{2}\right)_{!}\left(p r_{1}^{*} K \otimes \mathscr{L}_{\psi\left(e, e^{\vee}\right)}\right)[r] .
$$

1.7.1.2. One knows [ $\mathrm{Br}, 9.3]$ that $\mathrm{FT}_{\psi /}$ is essentially involutive in the sense that

$$
\mathrm{FT}_{\psi}\left(\mathrm{FT}_{\psi}(K)\right) \approx[e \mapsto-e]^{*} K(-r)
$$

and that $K \mapsto \mathrm{FT}_{\psi}(K)$ preserves semiperversity (because the natural "forget supports" map

$$
\mathrm{FT}_{\psi}(K) \rightarrow R\left(p r_{2}\right)_{*}\left(p r_{1}^{*} K \otimes \mathscr{L}_{\psi,\left(e, e^{\vee}\right)}\right)[r]
$$

is an isomorphism, cf. [KaLa, 2.4.1]).

1.7.2. So in order to prove the theorem, namely, that

$$
M:=R\left(p r_{2}\right)_{!}\left(p r_{1}^{*} L \otimes f_{\text {aff }}^{*} K\right)[n m]
$$

on $\operatorname{Aff} \operatorname{Maps}\left(\mathbb{A}^{n}, \mathbb{A}^{m}\right)$ is semiperverse, it suffices to show that its Fourier Transform $\mathrm{FT}_{\psi}(M)$ is semiperverse, where we consider $\operatorname{Aff} \operatorname{Maps}\left(\mathbb{A}^{n}, \mathbb{A}^{m}\right)$ as a vector bundle over $S=\operatorname{Spec}(k)$. This is a geometric statement, so we may and will suppose henceforth that $k$ is algebraically closed.

1.7.3. To carry this out, we will need to make explicit the dual of $\operatorname{Aff} \operatorname{Maps}\left(\mathbb{A}^{n}, \mathbb{A}^{m}\right)$. For this, use coordinates $(A, b)$ to view

$$
\begin{aligned}
\operatorname{Aff} \operatorname{Maps}\left(\mathbb{A}^{n}, \mathbb{A}^{m}\right) & =\operatorname{Lin} \operatorname{Maps}\left(\mathbb{A}^{n}, \mathbb{A}^{m}\right) \times \mathbb{A}^{m} \\
& =\operatorname{Lin} \operatorname{Maps}\left(\mathbb{A}^{n}, \mathbb{A}^{m}\right) \times \operatorname{Lin} \operatorname{Maps}\left(\mathbb{A}^{1}, \mathbb{A}^{m}\right)
\end{aligned}
$$

Its dual is then

$$
\operatorname{Lin} \operatorname{Maps}\left(\mathbb{A}^{m}, \mathbb{A}^{n}\right) \times \operatorname{Lin} \operatorname{Maps}\left(\mathbb{A}^{m}, \mathbb{A}^{1}\right)
$$

with coordinates $(W, z)$ and pairing

$$
(A, b) \times(W, z) \mapsto \operatorname{Tr}(A W+b z),
$$

where $\operatorname{Tr}$ is the trace function on $\operatorname{End}\left(\mathbb{A}^{m}\right)=\{m \times m$ matrices $\}$.

1.7.4. In the calculations to follow, certain subvarieties $\mathscr{R} \supset \mathscr{R}_{0} \supset \mathscr{R}^{*}$ of the space $\operatorname{Lin} \operatorname{Maps}\left(\mathbb{A}^{m}, \mathbb{A}^{n}\right) \times \operatorname{Lin} \operatorname{Maps}\left(\mathbb{A}^{m}, \mathbb{A}^{1}\right)$ will play an important role. To describe them, it will be convenient to think of the space $\operatorname{Lin} \operatorname{Maps}\left(\mathbb{A}^{m}, \mathbb{A}^{n}\right) \times$ $\operatorname{Lin} \operatorname{Maps}\left(\mathbb{A}^{m}, \mathbb{A}^{1}\right)$ as being the space $\operatorname{Lin} \operatorname{Maps}\left(\mathbb{A}^{m}, \mathbb{A}^{n+1}\right)$, i.e., to think of the point $(W, z)$ as being the $(n+1) \times m$ matrix obtained by concatenating $z$ to $W$ as bottom row.

1.7.5. We define $\mathscr{R}$ to be the closed subvariety of the space $\operatorname{Lin} \operatorname{Maps}\left(\mathbb{A}^{m}, \mathbb{A}^{n}\right)$ $\times \operatorname{Lin} \operatorname{Maps}\left(\mathbb{A}^{m}, \mathbb{A}^{1}\right)$ consisting of those points $(W, z)$ for which

$$
\operatorname{rank}(W, z) \leq 1 \text {. }
$$

1.7.6. We define $\mathscr{R}_{0}$ to be the constructible subset of $\mathscr{R}$ where

$$
\operatorname{rank}(W, z) \leq \operatorname{rank}(z)
$$

The geometric points of $\mathscr{R}_{0}$ are those $(W, z)$ such that each row of $W$ is proportional to $z$. 
1.7.7. We define $\mathscr{R}^{*}$ to be the open subvariety of $\mathscr{R}$ where

$$
\operatorname{rank}(z)=1 \text {. }
$$

Equivalently, $\mathscr{R}^{*}$ is defined by the conditions

$$
\operatorname{rank}(W, z)=\operatorname{rank}(z)=1 .
$$

1.8.1. Lemma. The map

$$
\mathbb{A}^{n} \times\left(\operatorname{Lin} \operatorname{Maps}\left(\mathbb{A}^{m}, \mathbb{A}^{1}\right)-\{0\}\right) \rightarrow \mathscr{R}^{*}, \quad(x, z \neq 0) \mapsto(x z, z)
$$

is an isomorphism.

Proof. The conditions

$$
\operatorname{rank}(W, z)=\operatorname{rank}(z)=1
$$

are precisely that some component of $z$ is invertible and that each row of $W$ is proportional to $z$. The point $x$ in $\mathbb{A}^{n}$ is simply the vector whose components are these factors of proportionality. Q.E.D.

\subsubsection{Lemma. For any situation such that}

$k$ is algebraically closed of characteristic $p \neq l$;

$V$ is a separated $k$-scheme of finite type;

$f: V \rightarrow \mathbb{A}^{m}$ is an arbitrary morphism;

$\pi: V \rightarrow \mathbb{A}^{n}$ is an arbitrary morphism;

$K$ is in $D_{c}^{b}\left(\mathbb{A}^{m}, \overline{\mathbb{Q}}_{l}\right)$;

$L$ is in $D_{c}^{b}\left(V, \overline{\mathbb{Q}}_{l}\right)$;

the object $M:=R\left(p r_{2}\right)_{!}\left(p r_{1}^{*} L \otimes f_{\text {aff }}^{*} K\right)[n m]$ has $\mathrm{FT}_{\psi}(M)$ supported in $\mathscr{R}_{0}$. If, in addition, either $H_{c}\left(\mathbb{A}^{m}, K\right)=0$ or $H_{c}(V, L)=0$, then $\mathrm{FT}_{\psi}(M)$ is supported in $\mathscr{R}^{*}$.

Proof. We first calculate punctually the Fourier Transform of $M$. We abbreviate $\operatorname{Aff} \operatorname{Maps}\left(\mathbb{A}^{n}, \mathbb{A}^{m}\right)$ as Aff. The stalk of $\operatorname{FT}_{\psi}(M)$ at $(W, z)$ is

$$
F \Gamma_{c}\left(\text { Aff, } M \otimes \mathscr{L}_{\psi(\operatorname{Tr}(A W+b z))}[n m+m]\right),
$$

which by the derived form of the Leray spectral sequence is

$$
=R \Gamma_{c}\left(V \times \text { Aff, } p r_{1}^{*} L \otimes f_{\text {aff }}^{*} K[n m] \otimes \mathscr{L}_{\psi(\operatorname{Tr}(A W+b z))}[n m+m]\right) .
$$

In terms of the automorphism $\sigma$ introduced above,

$$
\sigma:(v, A, b) \mapsto(v, A, b+A \pi(v)+f(v)),
$$

we may rewrite $p r_{1}^{*} L \otimes f_{\text {aff }}^{*} K$ as $\sigma^{*}\left(p r_{1}^{*} L \otimes p r_{3}^{*} K\right)$, so our stalk becomes

$$
\begin{aligned}
& =R \Gamma_{c}\left(V \times \operatorname{Lin} \times \mathbb{A}^{m}, \sigma^{*}\left(p r_{1}^{*} L \otimes p r_{3}^{*} K\right) \otimes \mathscr{L}_{\psi(\operatorname{Tr}(A W+b z))}[2 n m+m]\right) \\
& \approx R \Gamma_{c}\left(V \times \operatorname{Lin} \times \mathbb{A}^{m}, \sigma^{*}\left(p r_{1}^{*} L \otimes p r_{3}^{*} K\right) \otimes \sigma_{*} \mathscr{L}_{\psi(\operatorname{Tr}(A W+b z))}[2 n m+m]\right) \\
& =R \Gamma_{c}\left(V \times \operatorname{Lin} \times \mathbb{A}^{m}, p r_{1}^{*} L \otimes p r_{3}^{*} K \otimes N\right)[2 n m+m],
\end{aligned}
$$


where

$$
\begin{aligned}
N & :=\sigma_{*} \mathscr{L}_{\psi(\operatorname{Tr}(A W+b z))} \\
& :=\mathscr{L}_{\psi(\operatorname{Tr}(A W+b z-A \pi(v) z-f(v) z))} \\
& :=\mathscr{L}_{\psi(\operatorname{Tr}(A(W-\pi(v) z)))} \otimes \mathscr{L}_{\psi(\operatorname{Tr}(-f(v) z)) \otimes} \mathscr{L}_{\psi(\operatorname{Tr}(b z))} .
\end{aligned}
$$

By the Kunneth formula [SGA4, XVII, 5.4.3] for $R \Gamma_{c}$, we may rewrite our stalk as a tensor product $X \otimes Y$, with

$$
\begin{aligned}
& X:=R \Gamma_{c}\left(V \times \operatorname{Lin}, p r_{1}^{*} L \otimes \mathscr{L}_{\psi(\operatorname{Tr}(A(W-\pi(v) z))} \otimes \mathscr{L}_{\psi(\operatorname{Tr}(-f(v) z))}[2 n m]\right), \\
& Y:=R \Gamma_{c}\left(\mathbb{A}^{m}, K \otimes \mathscr{L}_{\psi(\operatorname{Tr}(b z))}[m]\right):=\mathrm{FT}_{\psi}(K)_{z} .
\end{aligned}
$$

We now show that the $X$ factor vanishes when we are at a point $(W, z)$, which is not in $\mathscr{R}_{0}$, i.e., one for which $W-x z \neq 0$ for any $x$ in $\mathbb{A}^{n}$. We will compute

$$
X:=R \Gamma_{c}\left(V \times \operatorname{Lin}, p r_{1}^{*} L \otimes \mathscr{L}_{\psi(\operatorname{tr}(A(W-\pi(v) z)))} \otimes \mathscr{L}_{\psi(\operatorname{Tr}(-f(v) z))}[2 n m]\right),
$$

by using the (derived form of the) Leray spectral sequence for the map

$$
p r_{1}: V \times \operatorname{Lin} \rightarrow V .
$$

By the projection formula, we have

$$
X=R \Gamma_{c}\left(V, L \otimes \mathscr{L}_{\psi(\operatorname{Tr}(-f(v) z))}[2 n m] \otimes R\left(p r_{1}\right)_{!}\left(\mathscr{L}_{\psi(\operatorname{Tr}(A(W-\pi(v) z)))}\right)\right) .
$$

Because $(W, z)$ is not in $\mathscr{R}$, we have $W-\pi(v) z \neq 0$ for any $v$ in $V$. Therefore for fixed $(v, W, z), A \mapsto \operatorname{Tr}(A(W-\pi(v) z))$ is a nontrivial linear form, and hence, looking fibre by fibre over $V$, we see that

$$
R\left(p r_{1}\right)_{!}\left(\mathscr{L}_{\psi(\operatorname{Tr}(A(W-\pi(v) z)))}\right)=0 .
$$

[Indeed, for any nontrivial linear form $\varphi$ on an affine space $E$, one has $R \Gamma_{c}\left(E, \mathscr{L}_{\psi(\varphi)}\right)=0$; by taking $\varphi$ as one of the coordinates in $E^{\vee}$ and the dual basis of $E$, one reduces by Kunneth to the case when $E$ is $\mathbb{A}^{1}$ and to the vanishing of $R \Gamma_{c}\left(\mathbb{A}^{1}, \mathscr{L}_{\psi}\right)$.] Thus we have proven that $\mathrm{FT}_{\psi}(M)$ is supported in $\mathscr{R}_{0}$.

The only geometric point of $\mathscr{R}_{0}$ not in $\mathscr{R}^{*}$ is $(0,0)$. At this point, $X$ becomes

$$
\begin{aligned}
X_{\mid(0,0)} & =R \Gamma_{c}\left(V \times \operatorname{Lin}, p r_{1}^{*} L\right)[2 n m] \\
& =R \Gamma_{c}(V, L) \otimes R \Gamma_{c}\left(\operatorname{Lin}, \overline{\mathbb{Q}}_{l}\right)[2 n m] \\
& =R \Gamma_{c}(V, L)(-n m)
\end{aligned}
$$

and $Y$ becomes

$$
Y_{\mid(0,0)}=R \Gamma_{c}\left(\mathbb{A}^{m}, K[m]\right)
$$

Thus at $(0,0)$, the stalk of our Fourier Transform is

$$
R \Gamma_{c}(V, L) \otimes R \Gamma_{c}\left(\mathbb{A}^{m}, K\right)[m](-n m),
$$

which visibly vanishes if either $R \Gamma_{c}(V, L)$ or $R \Gamma_{c}\left(\mathbb{A}^{m}, K\right)$ vanishes. Q.E.D. 
1.8.3. Lemma. With the notation of the lemma above, for any situation such that

$k$ is a field of characteristic $p \neq l$;

$V$ is a separated $k$-scheme of finite type;

$f: V \rightarrow \mathbb{A}^{m}$ is an arbitrary morphism;

$\pi: V \rightarrow \mathbb{A}^{n}$ is an arbitrary morphism;

$K$ is in $D_{c}^{b}\left(\mathbb{A}^{m}, \overline{\mathbb{Q}}_{l}\right)$;

$L$ is in $D_{c}^{b}\left(V, \overline{\mathbb{Q}}_{l}\right)$;

the restriction of $\mathrm{FT}_{\psi}(M)$ to $\mathscr{R}^{*} \approx \mathbb{A}^{n} \times\left(\operatorname{Lin} \operatorname{Maps}\left(\mathbb{A}^{m}, \mathbb{A}^{1}\right)-\{0\}\right)$, with coordinates $(x, z)$, is given as follows. On the product

$$
V \times\left(\operatorname{Lin} \operatorname{Maps}\left(\mathbb{A}^{m}, \mathbb{A}^{1}\right)-\{0\}\right),
$$

with coordinates $(v, z)$, consider the object

$$
p r_{1}^{*} L \otimes p r_{2}^{*} \mathrm{FT}_{\psi}(K) \otimes \mathscr{L}_{\psi(\operatorname{Tr}(-f(v) z))} .
$$

Consider the morphism

$$
\begin{aligned}
& V \times\left(\operatorname{Lin} \operatorname{Maps}\left(\mathbb{A}^{m}, \mathbb{A}^{1}\right)-\{0\}\right) \\
& \downarrow(\pi \times \text { id }) \\
& \mathbb{A}^{n} \times\left(\operatorname{Lin} \operatorname{Maps}\left(\mathbb{A}^{m}, \mathbb{A}^{1}\right)-\{0\}\right)
\end{aligned}
$$

We have

$$
\mathrm{FT}_{\psi}(M) \mid \mathscr{R}^{*} \approx R(\pi \times \mathrm{id})_{!}\left(p r_{1}^{*} L \otimes p r_{2}^{*} \mathrm{FT}_{\psi}(K) \otimes \mathscr{L}_{\psi(\operatorname{Tr}(-f(v) z))}\right)(-n m) .
$$

Proof. By proper base change and the projection formula, the restriction $\mathrm{FT}_{\psi}(M) \mid \mathscr{R}^{*}$ is given in terms of the map

$$
p r_{4}: V \times \operatorname{Lin} \times \mathbb{A}^{m} \times \mathscr{R}^{*} \rightarrow \mathscr{R}^{*}
$$

in coordinates $(v, A, b, x, z,) \mapsto(x, z)$ as

$$
R\left(p r_{4}\right)_{!}\left(p r_{1}^{*} L \otimes f_{\mathrm{aff}}^{*} K \otimes \mathscr{L}_{\psi(\operatorname{Tr}(A W+b z))}\right)[2 n m+m] .
$$

Remembering that $W=x z$ on $\mathscr{R}^{*}$, and using the automorphism $\sigma$ as we did in the punctual case above, we rewrite this as

$$
R\left(p r_{4}\right)_{!}\left(p r_{1}^{*} L \otimes p r_{3}^{*} K \otimes N\right)[2 n m+m],
$$

where $N:=\mathscr{L}_{\psi(\operatorname{Tr}(A(x-\pi(v)) z))} \otimes \mathscr{L}_{\psi(\operatorname{Tr}(-f(v) z))} \otimes \mathscr{L}_{\psi(\operatorname{Tr}(b z))}$. By factoring $p r_{4}$ through the projection onto $V \times \operatorname{Lin} \times \mathscr{R}^{*}$, which amounts to summing over $b$, we may rewrite this in terms of the morphism

$$
p r_{3,4}: V \times \operatorname{Lin} \times \mathscr{R}^{*} \rightarrow \mathscr{R}^{*}
$$

in coordinates $(v, A, x, z) \mapsto(x, z)$ as

$$
R\left(p r_{3,4}\right)_{!}\left(p r_{1}^{*} L \otimes \mathrm{FT}_{\psi}(K)_{z} \otimes \mathscr{L}_{\psi(\operatorname{Tr}(A(x-\pi(v)) z))} \otimes \mathscr{L}_{\psi(\operatorname{Tr}(-f(v) z))}\right)[2 n m] .
$$

The next step is to factor $p r_{3,4}$ through the projection $p r_{1,3,4}$ onto $V \times \mathscr{R}^{*}$, i.e., to sum over $A$. For this, we must calculate

$$
R\left(p r_{1,3,4}\right),\left(\mathscr{L}_{\psi(\operatorname{Tr}(A(X-\pi(v)) z))}\right)[2 n m] .
$$


By proper base change, this is the pullback by the map

$$
\begin{aligned}
V \times \mathscr{R}^{*} \rightarrow \operatorname{Lin}^{\vee}:=\operatorname{Lin} \operatorname{Maps}\left(\mathbb{A}^{m}, \mathbb{A}^{n}\right), \\
(v, x, z) \mapsto(x-\pi(v)) z
\end{aligned}
$$

of the Fourier Transform of the shifted constant sheaf $\overline{\mathbb{Q}}_{l}[\mathrm{~nm}]$ on Lin; but this Fourier Transform is the delta function $\overline{\mathbb{Q}}_{l, 0}(-n m)$ at the origin of $\operatorname{Lin}^{\vee}$. So if we denote by $\mathscr{Z}$ the closed subvariety of $V \times \mathscr{R}^{*}$ where the function $(x-\pi(v)) z$ vanishes,

$$
\mathscr{Z}:=\left\{(v, x, z) \text { in } V \times \mathscr{R}^{*} \text { where }(x-\pi(v)) z=0\right\},
$$

we have

$$
R\left(p r_{1,3,4}\right)_{!}\left(\mathscr{L}_{\psi(\operatorname{Tr}(A(x-\pi(v)) z))}\right)[2 n m] \approx \overline{\mathbb{Q}}_{l, \mathscr{Z}}(-n m)
$$

Denote by $p r_{2,3} \mid \mathscr{Z}$ the map

$$
p r_{2,3} \mid \mathscr{Z}: \mathscr{Z} \rightarrow \mathscr{R}^{*}, \quad(v, x, z) \text { in } \mathscr{Z} \rightarrow(x, z) .
$$

By the projection formula, we have

$$
\mathrm{FT}_{\psi}(M) \mid \mathscr{R}^{*} \approx R\left(p r_{2,3} \mid \mathscr{Z}\right)_{!}\left(p r_{1}^{*} L \otimes \mathrm{FT}_{\psi}(K)_{Z} \otimes \mathscr{L}_{\psi(\operatorname{Tr}(-f(v) z)))}\right)(-n m) .
$$

Since $z \neq 0$ in $\mathscr{R}^{*}$, the vanishing of the matrix $(x-\pi(v)) z$ is equivalent to the vanishing of the vector $x-\pi(v)$. In other words, the subvariety $\mathscr{Z}$ of

$$
V \times \mathscr{R}^{*} \approx V \times \mathbb{A}^{n} \times\left(\operatorname{Lin} \operatorname{Maps}\left(\mathbb{A}^{m}, \mathbb{A}^{1}\right)-\{0\}\right)
$$

is none other than the product with the third factor of the graph of the map $\pi: V \rightarrow \mathbb{A}^{n}:$

$$
\mathscr{Z}=\Gamma_{\pi} \times\left(\operatorname{Lin} \operatorname{Maps}\left(\mathbb{A}^{m}, \mathbb{A}^{1}\right)-\{0\}\right)
$$

If we identify the graph $\Gamma_{\pi}$ with its source $V$, then $\mathscr{Z}$ becomes the product $V \times\left(\operatorname{Lin} \operatorname{Maps}\left(\mathbb{A}^{m}, \mathbb{A}^{1}\right)-\{0\}\right)$,

$$
p r_{1}^{*} L \otimes \mathrm{FT}_{\psi}(K)_{z} \otimes \mathscr{L}_{\psi(\operatorname{Tr}(-f(v) z))} \quad \text { on } \mathscr{Z}
$$

becomes

$$
p r_{1}^{*} L \otimes p r_{2}^{*} \mathrm{FT}_{\psi}(K) \otimes \mathscr{L}_{\psi(\operatorname{Tr}(-f(v) z))} \quad \text { on } V \times\left(\operatorname{Lin} \operatorname{Maps}\left(\mathbb{A}^{m}, \mathbb{A}^{1}\right)-\{0\}\right),
$$

and the map $\operatorname{pr}_{2,3} \mid \mathscr{Z}: \mathscr{Z} \rightarrow \mathscr{R}^{*},(v, x, z)$ in $\mathscr{Z} \rightarrow(x, z)$ becomes the map

$$
\begin{aligned}
& V \times\left(\operatorname{Lin} \operatorname{Maps}\left(\mathbb{A}^{m}, \mathbb{A}^{1}\right)-\{0\}\right) \\
& \downarrow(\pi \times \text { id }):(v, z) \mapsto(\pi(v), z) \\
& \mathbb{A}^{n} \times\left(\operatorname{Lin} \operatorname{Maps}\left(\mathbb{A}^{m}, \mathbb{A}^{1}\right)-\{0\}\right) . \quad \text { Q.E.D. }
\end{aligned}
$$

With these lemmas, the proof of Theorem 1.5 is immediate. Under the hypotheses of the theorem, $\mathrm{FT}_{\psi}(M)$ is supported in $\mathscr{R}^{*}$ by Lemma 1.8.2. Since extension by zero preserves semiperversity, it suffices to show that $\mathrm{FT}_{\psi}(M)$ is semiperverse on $\mathscr{R}^{*}$. On $\mathscr{R}^{*}$ it is

$$
R(\pi \times \mathrm{id})_{!}\left(p r_{1}^{*} L \otimes p r_{2}^{*} \mathrm{FT}_{\psi}(K) \otimes \mathscr{L}_{\psi(\operatorname{Tr}(-f(v) z))}\right)(-n m) .
$$


Since $\pi$ is quasi-finite so is $\pi \times \mathrm{id}$, and so $R(\pi \times \mathrm{id})$, preserves semiperversity. It remains to show that

$$
\begin{aligned}
\left(p r_{1}^{*} L \otimes p r_{2}^{*} \mathrm{FT}_{\psi}(K) \otimes \mathscr{L}_{\psi(\operatorname{Tr}(-f(v) z))}\right)(-n m) & \\
& \text { on } V \times\left(\operatorname{Lin} \operatorname{Maps}\left(\mathbb{A}^{m}, \mathbb{A}^{1}\right)-\{0\}\right)
\end{aligned}
$$

is semiperverse.

Tensoring with a lisse sheaf, here $\mathscr{L}_{\psi(\operatorname{Tr}(-f(v) z))}(-n m)$, preserves semiperversity, so we are reduced to the semiperversity of the external tensor product $p r_{1}^{*} L \otimes p r_{2}^{*} \mathrm{FT}_{\psi}(K)$; but the external tensor product of semiperverse objects is semiperverse, so we are reduced to observing that $L$ is semiperverse on $V$ by hypothesis, $\mathrm{FT}_{\psi}(K)$ is semiperverse on (Lin Maps $\left.\left(\mathbb{A}^{m}, \mathbb{A}^{1}\right)-\{0\}\right)$ because $K$ is semiperverse on $\mathbb{A}^{m}$, and Fourier Transform preserves semiperversity. Q.E.D.

\section{RESULTS ON IRREDUCIBILITY AND PURITY}

\subsection{Irreducibility of the highest weight part.}

2.1.1. Theorem. Suppose that:

$k$ is a finite field of characteristic $p \neq l$;

$V$ is an affine $k$-scheme of finite type;

$f: V \rightarrow \mathbb{A}^{m}$ is an arbitrary morphism;

$\pi: V \rightarrow \mathbb{A}^{n}$ is a closed immersion;

$K$ is perverse on $\mathbb{A}^{m}$, geometrically nonconstant, geometrically irreducible, and pure of weight $\alpha$;

$L$ is perverse nonzero on $V$, geometrically irreducible, and pure of weight $\beta$;

and either $H_{c}\left(\mathbb{A}^{m} \otimes \bar{k}, K\right)=0$ or $H_{c}(V \otimes \bar{k}, L)=0$. Then:

(1) The perverse sheaf $M:=R\left(p r_{2}\right)_{1}\left(p r_{1}^{*} L \otimes f_{\text {aff }}^{*} K\right)[n m]$ on $\operatorname{Aff} \operatorname{Maps}\left(\mathbb{A}^{n}, \mathbb{A}^{m}\right)$ is mixed of weight $\leq \alpha+\beta+n m$ and is nonzero.

(2) Its highest weight quotient (of weight $=\alpha+\beta+n m$ ) as a perverse sheaf [BBD, 5.3.5] is geometrically irreducible.

(3) $M$ is geometrically irreducible if and only if $M$ is pure of weight $\alpha+$ $\beta+n m$.

Proof. That $M$ is perverse was proven in the Perversity Corollary 1.6. That $M$ is mixed of weight $\leq \alpha+\beta+n m$ follows from the main result of Deligne's Weil II [De, 3.3.1, 6.2.3].

We will prove (2) and (3) on the Fourier Transform side, where they become the statements that the highest weight quotient of $\mathrm{FT}_{\psi}(M)$ (of weight $=\alpha+\beta+$ $2 n m+m)$ is geometrically irreducible as a perverse sheaf on $\operatorname{Aff} \operatorname{Maps}\left(\mathbb{A}^{n}, \mathbb{A}^{m}\right)$ and that $\mathrm{FT}_{\psi}(M)$ is geometrically irreducible if and only if it is pure of weight $\alpha+\beta+2 n m+m$. 
We have already proven that $\mathrm{FT}_{\psi}(M)$ is supported in the open subvariety $\mathscr{R}^{*}$ of the closed subvariety $\mathscr{R}$ of $\operatorname{Aff} \operatorname{Maps}\left(\mathbb{A}^{n}, \mathbb{A}^{m}\right)$. Let us denote by $j: \mathscr{R}^{*} \rightarrow \mathscr{R}$ and $i: \mathscr{R} \rightarrow \operatorname{Aff} \operatorname{Maps}\left(\mathbb{A}^{n}, \mathbb{A}^{m}\right)$ the inclusions. In terms of these, we have proven that

$$
\mathrm{FT}_{\psi}(M)=i_{*} j_{!} j^{*} i^{*} \mathrm{FT}_{\psi}(M) .
$$

Now $i_{*}$ carries perverse irreducibles to perverse irreducibles and preserves the filtration by the weight, so $j_{!} j^{*} i^{*} \mathrm{FT}_{\psi}(M)$ is perverse on $\mathscr{R}$, and it suffices to show that the highest weight quotient of $j_{!} j^{*} i^{*} F T_{\psi}(M)$ (of weight $\alpha+\beta+$ $2 n m+m)$ is geometrically irreducible as a perverse sheaf on $\mathscr{R}$.

We first claim that $j^{*} i^{*} \mathrm{FT}_{\psi}(M)$ on $\mathscr{R}^{*}$ is perverse, geometrically irreducible, and pure of weight $\alpha+\beta+2 \mathrm{~nm}+m$. To see this, we argue as follows. Because the map $\pi: V \rightarrow \mathbb{A}^{n}$ is a closed immersion, Lemma 1.8.3 shows that on $\mathscr{R}^{*} \approx \mathbb{A}^{n} \times\left(\operatorname{Lin} \operatorname{Maps}\left(\mathbb{A}^{m}, \mathbb{A}^{1}\right)-\{0\}\right), j^{*} i^{*} \mathrm{FT}_{\psi}(M)$ is the extension by zero from $V \times\left(\operatorname{Lin} \operatorname{Maps}\left(\mathbb{A}^{m}, \mathbb{A}^{1}\right)-\{0\}\right)$, with coordinates $(v, z)$, of the object

$$
p r_{1}^{*} L \otimes p r_{2}^{*} \mathrm{FT}_{\psi}(K) \otimes \mathscr{L}_{\psi(\operatorname{Tr}(-f(v) z))}(-n m) .
$$

So it suffices to show that this object on

$$
V \times\left(\operatorname{Lin} \operatorname{Maps}\left(\mathbb{A}^{m}, \mathbb{A}^{1}\right)-\{0\}\right)
$$

is perverse, geometrically irreducible, and pure of weight $\alpha+\beta+2 n m+m$. Since $\mathscr{L}_{\psi(\operatorname{Tr}(-f(v) z))}$ is lisse of rank one and pure of weight zero, tensoring with it does not alter questions of perversity, purity of given weight, or geometric irreducibility. So it suffices to treat the object $p r_{1}^{*} L \otimes p r_{2}^{*} \mathrm{FT}_{\psi}(K)(-n m)$. This is the external tensor product of $L(-n m)$, which is perverse nonzero, geometrically irreducible, and pure of weight $\alpha+2 n m$ on $V$, with $\mathrm{FT}_{\psi}(K)$, which is perverse nonzero (because $K$ is geometrically nonconstant), geometrically irreducible, and pure of weight $\alpha+m$ on $\left(\operatorname{Lin} \operatorname{Maps}\left(\mathbb{A}^{m}, \mathbb{A}^{1}\right)-\{0\}\right)$. An external tensor product of perverse nonzero, geometrically irreducible, and pure objects is itself perverse nonzero, geometrically irreducible, and pure of weight the sum of the weights.

To conclude the proof of the theorem, we apply the following general lemma to the situation $X:=\mathscr{R}, U:=\mathscr{R}^{*}$ and to the object $N:=j^{*} i^{*} \mathrm{FT}_{\psi}(M)$ on $U$.

\subsubsection{Lemma. Suppose we are given}

$k$, a finite field of characteristic $p \neq l$;

$X$, a separated $k$-scheme of finite type;

$j: U \rightarrow X$, an open immersion;

$N$ in $D_{c}^{b}\left(U, \overline{\mathbb{Q}}_{I}\right)$, a perverse sheaf, which is pure of weight $w$, and such that $j_{1} N$ is perverse on $X$.

Then:

(1) The weight $w$ quotient of $j_{1} N$ is the middle extension $j_{1_{*}} N$, which is perverse and pure of weight $w$. Moreover, $j_{!} N \cong j_{!_{*}} N$ if and only if $j_{!} N$ is pure of weight $w$. 
(2) If, in addition, $N$ is nonzero and geometrically irreducible on $U$, then the middle extension $j_{!_{*}} N$ is perverse nonzero, geometrically irreducible, and pure of weight $w$. Moreover, $j_{1} N$ is geometrically irreducible if and only if it is pure of weight $w$.

Proof. For any open immersion $j: U \rightarrow X$ and any perverse $N$ on $U$, the middle extension $j_{t_{*}} N$ is defined [BBD, 1.4.22] in terms of the perverse cohomology sheaves ${ }^{p} \mathscr{H}^{0}\left(j_{1} N\right)$ and ${ }^{p} \mathscr{H}^{0}\left(R j_{*} N\right) \quad\left({ }^{p} j_{1} N\right.$ and ${ }^{p} j_{*} N$ in the notation of [BBD, 1.4.15]) as the image of the canonical map

$$
j_{! *} N:=\operatorname{Image}\left({ }^{p} \mathscr{\ell}^{0}\left(j_{!} N\right) \rightarrow{ }^{p} \mathscr{\ell}^{0}\left(R j_{*} N\right)\right) .
$$

In our situation, $j_{!} N$ is perverse by hypothesis, so we may rewrite the middle extension as

$$
j_{! *} N:=\operatorname{Image}\left(j_{!} N \rightarrow{ }^{p} \mathscr{H}^{0}\left(R j_{*} N\right)\right) .
$$

The key point is that this description shows that there is a canonical surjective map of perverse sheaves on $X$

$$
j_{!} N \rightarrow j_{! *} N .
$$

Now in $D_{c}^{b}\left(X, \overline{\mathbb{Q}}_{l}\right)$, if we denote by $Z:=X-U, i: Z \rightarrow X$ the inclusion, then we have a tautological triangle

$$
0 \rightarrow i_{*} i^{*} j_{! *} N[-1] \rightarrow j_{!} N \rightarrow j_{! *} N \rightarrow 0 .
$$

Since the map $j_{1} N \rightarrow j !_{*} N$ is a surjection of perverse sheaves, it follows from [BBD, 1.2.2, 1.2.3] that $i_{*} i^{*} j_{t_{*}} N[-1]$ is perverse and that

$$
0 \rightarrow i_{*} i^{*} j_{! *} N[-1] \rightarrow j_{!} N \rightarrow j_{! *} N \rightarrow 0
$$

is a short exact sequence of perverse sheaves on $X$.

By [BBD, 5.4.3], $j_{1 *} N$ is pure of weight $w$. Therefore $i_{*} j^{*} j_{t_{*}} N$ is mixed of weight $\leq w$; hence, $i_{*} i^{*} j_{1 *} N[-1]$ is mixed of weight $\leq w-1$. This shows that $j_{1 *} N$ is indeed the weight $w$ quotient of $j_{1} N$. Therefore the map $j_{1} N \rightarrow j_{1 *} N$ is an isomorphism if and only if $j_{1} N$ is pure of weight $w$. This proves (1).

To prove (2), suppose now that $N$ is perverse nonzero and geometrically irreducible on $U$. It follows form the explicit description of irreducibles [BBD, 4.3.1] that $j_{1 *} N$ is perverse and geometrically irreducible on $X$. If $j_{1} N$ is pure of weight $w$, the fact that $j_{! *} N$ is its weight $w$ quotient shows that $j_{!} N \approx j_{! *} N$ and, hence, that $j_{1} N$ is geometrically irreducible. Conversely, since $N$ is perverse nonzero, $j_{t_{*}} N$ is nonzero. So if $j_{!} N$ is geometrically irreducible, the map $j_{1} N \rightarrow j_{t_{*}} N$ must be an isomorphism, whence $j_{1} N$ is pure of weight $w$. Q.E.D.

2.1.3. Corollary. Hypotheses and notation are as in Theorem 2.1.1. Suppose that there exists a dense open set $U$ of $\mathrm{Aff} \operatorname{Maps}\left(\mathbb{A}^{n}, \mathbb{A}^{m}\right)$ on which $M:=$ $R\left(p r_{2}\right)_{!}\left(p r_{1}^{*} L \otimes f_{\mathrm{aff}}^{*} K\right)[\mathrm{nm}]$ is pure of weight $\alpha+\beta+n m$. Then $M \mid U$ is geometrically irreducible as a perverse sheaf on $U$.

Proof. Let us denote $w:=\alpha+\beta+n m, M(w)$ the weight $w$ quotient of $M$. Because $M(w)$ is the highest weight quotient of $M$, by [BBD, 5.3.5] we have a short exact sequence of perverse sheaves

$$
0 \rightarrow M(<w) \rightarrow M \rightarrow M(w) \rightarrow 0
$$


on $\operatorname{Aff} \operatorname{Maps}\left(\mathbb{A}^{n}, \mathbb{A}^{m}\right)$, with $M(<w)$ mixed of weight $<w$. Formation of this weight-filtration short exact sequence commutes with étale localization. Pulled back to $U$, it gives

$$
0 \rightarrow M(<w)|U \rightarrow M| U \rightarrow M(w) \mid U \rightarrow 0 .
$$

Since $M \mid U$ is pure of weight $w$, we have

$$
M|U \cong M(w)| U .
$$

By Theorem 2.1.1, $M(w)$ is geometrically irreducible. By the known structure [BBD, 4.3.1] of perverse irreducibles, $M(w) \mid U$ remains geometrically irreducible. Q.E.D.

2.1.4. Corollary. Hypotheses and notation are as in Theorem 2.1.1. Suppose that there exists a dense open set $U$ of $\operatorname{Aff} \operatorname{Maps}\left(\mathbb{A}^{n}, \mathbb{A}^{m}\right)$ on which $M:=$ $\left(R\left(p r_{2}\right)_{!}\left(p r_{1}^{*} L \otimes f_{\mathrm{aff}}^{*} K\right)[n m]\right.$ is lisse, i.e., $M \mid U \approx \mathscr{F}[\mathrm{nm}+m]$ with $\mathscr{F}$ a lisse sheaf on $U$, mixed of weight $\leq \alpha+\beta-m$. Then the highest weight quotient $\mathscr{F}$ (weight $=\alpha+\beta-m)$ of $\mathscr{F}$ in the sense of [De, 3.4.1(ii)] is geometrically irreducible as a lisse sheaf on $U$.

Proof. Just as in the proof of 2.1.3, denote by $M(w)$ the highest weight quotient of $M ; M(w)$ is geometrically irreducible by 2.1 .1 and so $M(w) \mid U$ is geometrically irreducible on $U$. But $M(w) \mid U$ is the highest weight quotient of $M \mid U \approx \mathscr{F}[\mathrm{nm}+\mathrm{m}]$, and this highest weight quotient is

$$
\mathscr{F}(\text { weight }=\alpha+\beta-m)[n m+m] \text {. }
$$

Thus the perverse sheaf $\mathscr{F}$ (weight $=\alpha+\beta-m)[n m+m]$ on $U$ is geometrically irreducible on $U$, and hence the lisse sheaf $\mathscr{F}$ (weight $=\alpha+\beta-m$ ) on $U$ is geometrically irreducible as a lisse sheaf. Q.E.D.

2.2. An example and a question. Here is the motivating example. Take $n=$ $m=1, V=\mathbb{A}^{1}, \pi$ the identity map, $f: \mathbb{A}^{1} \rightarrow \mathbb{A}^{1}$ a polynomial $f(x)$ in one variable of degree $d \geq 2, \chi$ a nontrivial character of $k^{\times}, L$ the sheaf $\overline{\mathbb{Q}}_{l}[1]$ on $V=\mathbb{A}^{1}$, and $K$ the sheaf $j_{!} \mathscr{L}_{\chi}[1]$ on $\mathbb{A}^{1}$. If we view $\operatorname{Aff} \operatorname{Maps}\left(\mathbb{A}^{1}, \mathbb{A}^{1}\right)$ as the $\mathbb{A}^{2}$ with coordinates $(a, b)$ corresponding to $x \mapsto a x+b$ and write $f_{a, b}$ for the polynomial

$$
f_{a, b}(x):=f(x)+a x+b,
$$

then $M:=R\left(p r_{2}\right)_{!}\left(p r_{1}^{*} L \otimes f_{\text {aff }}^{*} K\right)[1]$ has

$$
\mathscr{H}^{i}(M)_{a, b}=H_{c}^{i+3}\left(\mathbb{A}^{1}\left[1 /\left(f_{a, b}\right)\right] \otimes \bar{k}, \mathscr{L}_{\chi\left(f_{a, b}\right)}\right) .
$$

The open set $U$ of $(a, b)$-space where $f_{a, b}$ has all distinct zeros is nonempty (cf. 3.5). On $U$, the object $M$ has lisse cohomology sheaves [La, 2.1.4] and

$$
\begin{gathered}
\mathscr{H}^{i}(M) \mid U=0 \text { for } 1 \neq-2, \\
\mathscr{H}^{-2}(M) \mid U \text { has rank } d-1 .
\end{gathered}
$$

Moreover, if $\chi^{d}$ is nontrivial, then $\mathscr{H}^{-2}(M) \mid U$ is punctually pure of weight 1 . This follows from the explicit description above of $\mathscr{H}^{i}(M)_{a, b}$ and [De, 3.2.3], 
for if we denote by

$$
j: \mathbb{A}^{1}\left[1 /\left(f_{a, b}\right)\right] \rightarrow \mathbb{P}^{1}
$$

the inclusion, then for $(a, b)$ in $U$, we have

$$
j \mathscr{L}_{\chi}\left(f_{a, b}\right)=j_{*} \mathscr{L}_{\chi\left(f_{a, b}\right)} \text {. }
$$

So by Corollary $2.1 .3, \mathscr{H}^{-2}(M) \mid U$ as a lisse sheaf on $U$ is geometrically irreducible, provided that $\chi^{d}$ is nontrivial.

In $\S 2.4$, we will show that for all $\chi$ of sufficiently large order, the perverse sheaf $M$ on $\operatorname{Aff} \operatorname{Maps}\left(\mathbb{A}^{1}, \mathbb{A}^{1}\right)$ is geometrically irreducible. Does this hold already for any $\chi$ with $\chi^{d}$ nontrivial?

2.3. Purity is for a reason. The sense of the following proposition is that, under fairly mild conditions, one can only achieve purity for the "standard reason" that an $R f_{1}$ maps isomorphically to an $R f_{*}$.

\subsubsection{Proposition. Suppose that}

$k$ is a finite field of characteristic $p \neq l$;

$V$ is an affine $k$-scheme of finite type;

$f: V \rightarrow \mathbb{A}^{m}$ is an arbitrary morphism;

$\pi: V \rightarrow \mathbb{A}^{n}$ is a finite morphism;

$K$ is perverse on $\mathbb{A}^{m}$ and pure of weight $\alpha$;

$L$ is perverse on $V$ and pure of weight $\beta$;

and either $H_{c}\left(\mathbb{A}^{m} \otimes \bar{k}, K\right)=H_{c}\left(\mathbb{A}^{m} \otimes \bar{k}, D K\right)=0$ or $H_{c}(V \otimes \bar{k}, L)=$ $H_{c}(V \otimes \bar{k}, D L)=0$. Denote by $M$ and $M^{*}$ the perverse sheaves on $\operatorname{Aff} \operatorname{Maps}\left(\mathbb{A}^{n}, \mathbb{A}^{m}\right)$

$$
\begin{aligned}
M & :=R\left(p r_{2}\right)_{!}\left(p r_{1}^{*} L \otimes f_{\mathrm{aff}}^{*} K\right)[n m] \\
M^{*}: & :=R\left(p r_{2}\right)_{!}\left(p r_{1}^{*} D L \otimes f_{\mathrm{aff}}^{*} D K\right)[n m](n m) .
\end{aligned}
$$

The following conditions are equivalent.

(1) $M$ is pure of weight $\alpha+\beta+n m$ and $M^{*}$ is pure of weight $-\alpha-\beta-n m$.

(2) The natural "forget supports" map is an isomorphism

$$
R\left(p r_{2}\right)_{!}\left(p r_{1}^{*} L \otimes f_{\mathrm{aff}}^{*} K\right)[n m] \rightarrow R\left(p r_{2}\right)_{*}\left(p r_{1}^{*} L \otimes f_{\mathrm{aff}}^{*} K\right)[n m] .
$$

(3) The natural "forget supports" map is an isomorphism

$$
\begin{aligned}
& R\left(p r_{2}\right)_{!}\left(p r_{1}^{*} D L \otimes f_{\mathrm{aff}}^{*} D K\right)[n m](n m) \\
& \quad \rightarrow R\left(p r_{2}\right)_{*}\left(p r_{1}^{*} D L \otimes f_{\mathrm{aff}}^{*} D K\right)[n m](n m) .
\end{aligned}
$$

(4) The natural pairing makes $M$ and $M^{*}$ Verdier duals of each other.

Proof. Statements (2) and (3) are equivalent to each other, being duals of each other, and by duality and biduality each is equivalent to (4).

We first show that $(4) \Rightarrow(1)$. By [De, 3.3.1], $M$ is mixed of weight $\leq$ $\alpha+\beta+n m$, and $M^{*}$ is mixed of weight $\leq-\alpha-\beta-n m$. So if $M \cong D\left(M^{*}\right)$ and $M^{*} \cong D M$, then (1) holds by definition of purity. 
It remains to show that $(1) \Rightarrow(4)$. We will do this by once again making use of the Fourier Transform. We make use of the notation of the proof of Theorem 2.1.1. On $U:=\mathscr{R}^{*}$ we consider the objects

$$
N:=j^{*} i^{*} \mathrm{FT}_{\psi}(M) \text { and } N^{*}:=j^{*} i^{*} \mathrm{FT}_{\bar{\psi}}\left(M^{*}\right) \text {. }
$$

Lemma 1.8.3 shows that on $\mathscr{R}^{*} \approx \mathbb{A}^{n} \times\left(\operatorname{Lin} \operatorname{Maps}\left(\mathbb{A}^{m}, \mathbb{A}^{1}\right)-\{0\}\right)$ we have the explicit description of $N$ and $N^{*}$ in terms of the objects $\mathscr{N}$ and $\mathscr{N}^{*}$ on $V \times\left(\operatorname{Lin} \operatorname{Maps}\left(\mathbb{A}^{m}, \mathbb{A}^{1}\right)-\{0\}\right)$, with coordinates $(v, z)$,

$$
\begin{aligned}
& \mathscr{N}:=p r_{1}^{*} L \otimes p r_{2}^{*} \mathrm{FT}_{\psi}(K) \otimes \mathscr{L}_{\psi(\operatorname{Tr}(-f(v) z))}(-n m), \\
& \mathscr{N}^{*}:=p r_{1}^{*} D L \otimes p r_{2}^{*} \mathrm{FT}_{\bar{\psi}}(D K) \otimes \mathscr{L}_{\bar{\psi}(\operatorname{Tr}(-f(v) z))},
\end{aligned}
$$

by the formulas

$$
N=R(\pi \times \mathrm{id}) ! \mathcal{N}, \quad N^{*}=R(\pi \times \mathrm{id}) \mathscr{N}^{*} .
$$

From the explicit description of the objects $\mathscr{N}$ and $\mathscr{N}^{*}$, we see that $\mathscr{N}$ is perverse and pure of weight $\alpha+\beta+m+2 n m$, that $\mathscr{N}^{*}$ is perverse and pure of weight $-\alpha-\beta+m$, and that $D \mathscr{N} \cong \mathscr{N}^{*}(n m+m)$. [Recall [KaLa, 2.1.5] that on an $\mathbb{A}^{m}$, duality $D$ interchanges $\mathrm{FT}_{\psi}(m / 2)$ and $\mathrm{FT}_{\bar{\psi}}(m / 2)$ and that $\left.\mathrm{FT}_{\psi}(m / 2) \circ \mathrm{FT}_{\bar{\psi}}(m / 2)=\mathrm{id}.\right]$

Because the morphism $\pi$ is finite, the morphism $\pi \times$ id is also finite, so $R(\pi \times \mathrm{id})_{1}=R(\pi \times \mathrm{id})_{*}$ is self dual and, consequently, $R(\pi \times \mathrm{id})$, preserves both perversity and purity of given weight. Therefore the object $N$ is pure of weight $\alpha+\beta+m+2 n m$ and $D N \cong N^{*}(n m+m)$.

We have seen (Lemma 2.1.2) that if $M$ (resp. $M^{*}$ ) is pure of weight $\alpha+\beta+$ $n m$ (resp. $-\alpha-\beta-n m)$, the canonical map $j_{!} N \rightarrow j_{! *} N$ (resp. $j_{!} N^{*} \rightarrow j_{! *} N^{*}$ ) is an isomorphism. So if $M$ and $M^{*}$ are pure of these weights, the dual of the isomorphism $j_{!} N^{*} \cong j_{l_{*}} N^{*}$ is (the Tate twist of) an isomorphism $j_{l_{*}} N \cong$ $R j_{*} N$, which combines with $j_{!} N \cong j_{! *} N$ to give an isomorphism $j_{!} N \cong R j_{*} N$. By duality, we may rewrite this as

$$
j_{!} N \cong D j_{!} D N=D\left(j_{!} N^{*}(m+n m)\right)
$$

or, equivalently, as

$$
j_{!} N(m+n m) \cong D j_{!} N^{*}
$$

Taking extension by zero to $\operatorname{Aff} \operatorname{Maps}\left(\mathbb{A}^{n}, \mathbb{A}^{m}\right)$, this says

$$
\mathrm{FT}_{\psi} M(m+n m) \cong D \mathrm{FT}_{\bar{\psi}} M^{*}=\mathrm{FT}_{\psi}\left(D M^{*}\right)(m+n m)
$$

Applying the Fourier Transform $\mathrm{FT}_{\bar{\psi}}$, we get the required isomorphism $M \cong$ $D\left(M^{*}\right)$. Q.E.D.

2.3.2. Question. Suppose that $K$ is perverse on $\mathbb{A}^{m}$, geometrically nonconstant, and pure of some weight $\alpha$. Are the conditions

$$
H_{c}\left(\mathbb{A}^{m} \otimes \bar{k}, K\right)=0, \quad H_{c}\left(\mathbb{A}^{m} \otimes \bar{k}, D K\right)=0
$$

in fact equivalent? Similarly, for $L$ perverse on $V$ and pure of weight $\beta$, are the two conditions

$$
H_{c}(V \otimes \bar{k}, L)=0, \quad H_{c}(V \otimes \bar{k}, D L)=0
$$


equivalent? More generally, for $L$ perverse and pure on $V$, is it true that

$$
\sum_{i} \operatorname{dim} H_{c}^{i}(V \otimes \bar{k}, L)=\sum_{i} \operatorname{dim} H_{c}^{i}(V \otimes \bar{k}, D L) ?
$$

If we drop the purity hypothesis, both of these can be false. For an example, denote by $j: \mathbb{G}_{m} \rightarrow \mathbb{A}^{1}$ the inclusion and take $K:=R j_{*} \overline{\mathbb{Q}}_{l}[1]$. Then $K$ is perverse on $\mathbb{A}^{1}$ and $H_{c}\left(\mathbb{A}^{1} \otimes \bar{k}, K\right)=0$, but the dual $D K$ is $j_{!} \overline{\mathbb{Q}}_{l}[1](1)$, for which

$$
H_{c}\left(\mathbb{A}^{1} \otimes \bar{k}, D K\right)=H_{c}\left(\mathbb{G}_{m} \otimes \bar{k}, \overline{\mathbb{Q}}_{l}[1]\right)(1)
$$

is nonzero.

\subsection{Theorem of irreducibility with multiplicative characters of sufficiently high order.}

2.4.1. Theorem. Suppose we are given:

$k$, an algebraically closed field of characteristic $p \neq l$;

$V$, an affine $k$-scheme of finite type;

$f: V \rightarrow \mathbb{A}^{m}$, an arbitrary morphism;

$\pi: V \rightarrow \mathbb{A}^{n}$, a closed immersion;

$K_{m-1}$, perverse irreducible on $\mathbb{A}^{m-1}$;

$L$, perverse irreducible on $V$.

For $\chi$ any nontrivial multiplicative character of a finite subfield of $k$, consider the Kummer sheaf $\mathscr{L}_{\chi}$ on $\mathbb{G}_{m}$ and its extension by zero $j_{!} \mathscr{L}_{\chi}$ to $\mathbb{A}^{1}$. Denote by $K(\chi)$ the external tensor product

$$
K(\chi):=p r_{1}^{*} K_{m-1} \otimes p r_{2}^{*} j_{!} \mathscr{L}_{\chi}[1] \quad \text { on } \mathbb{A}^{m}=\mathbb{A}^{m-1} \times \mathbb{A}^{1}
$$

Then:

(1) $K(\chi)$ is perverse irreducible on $\mathbb{A}^{m}$ and $H_{c}\left(\mathbb{A}^{m}, K(\chi)\right)=0$.

(2) There exists an integer $N_{0}$, depending on $\left(k, V, f, \pi, K_{m-1}, L\right)$, such that if the order of $\chi$ is $>N_{0}$, then the perverse sheaf

$$
M(\chi):=R\left(p r_{2}\right)_{!}\left(p r_{1}^{*} L \otimes f_{\mathrm{aff}}^{*} K(\chi)\right)[n m]
$$

on $\operatorname{Aff} \operatorname{Maps}\left(\mathbb{A}^{n}, \mathbb{A}^{m}\right)$ is irreducible.

(3) If the order of $\chi$ is $>N_{0}$, then the "forget supports" map is an isomorphism

$$
R\left(p r_{2}\right)_{!}\left(p r_{1}^{*} L \otimes f_{\mathrm{aff}}^{*} K(\chi)\right)[n m] \approx R\left(p r_{2}\right)_{*}\left(p r_{1}^{*} L \otimes f_{\mathrm{aff}}^{*} K(\chi)\right)[n m] .
$$

(4) For any single nontrivial $\chi$, if the "forget supports" map is an isomorphism

$$
R\left(p r_{2}\right)_{!}\left(p r_{1}^{*} L \otimes f_{\mathrm{aff}}^{*} K(\chi)\right)[n m] \approx R\left(p r_{2}\right)_{*}\left(p r_{1}^{*} L \otimes f_{\mathrm{aff}}^{*} K(\chi)\right)[n m],
$$

then the perverse sheaf

$$
M(\chi):=R\left(p r_{2}\right)_{!}\left(p r_{1}^{*} L \otimes f_{\text {aff }}^{*} K(\chi)\right)[n m] \quad \text { on } \operatorname{Aff} \operatorname{Maps}\left(\mathbb{A}^{n}, \mathbb{A}^{m}\right)
$$

is irreducible. 
Proof. Statement (1) is "mise pour memoire." The external tensor product of perverse irreducibles is perverse irreducible, and the vanishing of $H_{c}\left(\mathbb{A}^{m}, K(\chi)\right)$ results by Kunneth from the well-known vanishing of $H_{c}\left(\mathbb{A}^{1}, j \mathscr{L}_{\chi}\right)=$ $H_{c}\left(\mathbb{G}_{m}, \mathscr{L}_{\chi}\right)$. Indeed, if $\chi$ has order $d>1$, and if we denote by $[d]: \mathbb{G}_{m} \rightarrow \mathbb{G}_{m}$ the $d$ th power map, then

$$
[d]_{*} \overline{\mathbb{Q}}_{l}=\overline{\mathbb{Q}}_{l} \oplus \mathscr{L}_{\chi} \oplus \text { other terms, }
$$

so

$$
\begin{aligned}
H_{c}\left(\mathbb{G}_{m}, \overline{\mathbb{Q}}_{l}\right) & =H_{c}\left(\mathbb{G}_{m},[d]_{*} \overline{\mathbb{Q}}_{l}\right) \\
& =H_{c}\left(\mathbb{G}_{m}, \overline{\mathbb{Q}}_{l}\right) \oplus H_{c}\left(\mathbb{G}_{m}, \mathscr{L}_{\chi}\right) \oplus \text { other terms. }
\end{aligned}
$$

Comparing dimensions shows that $H_{c}\left(\mathbb{G}_{m}, \mathscr{L}_{\chi}\right)=0$.

Having proven (1), we know from the Perversity Corollary 1.6 that $M(\chi)$ is indeed perverse. We will prove (2) by working on the Fourier Transform side, i.e., by showing that $\mathrm{FT}_{\psi}(M(\chi))$ is perverse irreducible for $\chi$ of sufficiently high order.

Just as in the proof of Theorem 2.1.1, we know that $\mathrm{FT}_{\psi}(M(\chi))$ is supported in $\mathscr{R}^{*} \approx \mathbb{A}^{n} \times\left(\operatorname{Lin} \operatorname{Maps}\left(\mathbb{A}^{m}, \mathbb{A}^{1}\right)-\{0\}\right)$, where it is the extension by zero from $V \times\left(\operatorname{Lin} \operatorname{Maps}\left(\mathbb{A}^{m}, \mathbb{A}^{1}\right)-\{0\}\right)$, with coordinates $(v, z)$, of the object

$$
p r_{1}^{*} L \otimes p r_{2}^{*} \mathrm{FT}_{\psi}(K(\chi)) \otimes \mathscr{L}_{\psi(\operatorname{Tr}(-f(v) z))}(-n m) .
$$

Now consider in greater detail the object $\mathrm{FT}_{\psi}(K(\chi))$ on $\operatorname{Lin} \operatorname{Maps}\left(\mathbb{A}^{m}, \mathbb{A}^{1}\right)$. In terms of the factorization

$$
\mathbb{A}^{m}=\mathbb{A}^{m-1} \times \mathbb{A}^{1}, \quad \text { coordinates }\left(x_{1}, \ldots, x_{m}\right)
$$

we get the dual factorization

$$
\operatorname{Lin} \operatorname{Maps}\left(\mathbb{A}^{m}, \mathbb{A}^{1}\right)=\operatorname{Lin} \operatorname{Maps}\left(\mathbb{A}^{m-1}, \mathbb{A}^{1}\right) \times \operatorname{Lin} \operatorname{Maps}\left(\mathbb{A}^{1}, \mathbb{A}^{1}\right),
$$

with corresponding coordinates $\left(z_{1}, \ldots, z_{m}\right)$.

The Fourier Transform $\mathrm{FT}_{\psi}(K(\chi))$ is itself the external tensor product

$$
\mathrm{FT}_{\psi}(K(\chi))=\mathrm{FT}_{\psi}\left(K_{m-1}\right) \times \mathrm{FT}_{\psi}\left(j \mathscr{L}_{\chi}[1]\right) \quad \text { on } \mathbb{A}^{m}=\mathbb{A}^{m-1} \times \mathbb{A}^{1},
$$

and $\mathrm{FT}_{\psi}\left(j_{1} \mathscr{L}_{\chi}[1]\right)$ is given geometrically by

$$
\mathrm{FT}_{\psi}\left(j, \mathscr{L}_{\chi}[1]\right)=j ! \mathscr{L}_{\bar{\chi}}[1]
$$

Therefore $\mathrm{FT}_{\psi}(K(\chi))$ vanishes at any point $z$ with $z_{m}=0$.

Thus we see that $\mathrm{FT}_{\psi}(M(\chi))$ is supported in the open set $\mathscr{R}\left[1 / z_{m}\right]$ of $\mathscr{R}$ (or equivalently of $\mathscr{R}^{*}$ ) where $z_{m}$ is invertible. On the open set $\mathscr{R}\left[1 / z_{m}\right]$, FT $_{\psi}(M(\chi))$ is perverse irreducible, being

$$
p r_{1}^{*} L \otimes p r_{2}^{*} \mathrm{FT}_{\psi}(K(\chi)) \otimes \mathscr{L}_{\psi(\operatorname{Tr}(-f(v) z))}(-n m)
$$

If we view this as living on

$$
V \times \operatorname{Lin} \operatorname{Maps}\left(\mathbb{A}^{m-1}, \mathbb{A}^{1}\right) \times\left(\operatorname{Lin} \operatorname{Maps}\left(\mathbb{A}^{1}, \mathbb{A}^{1}\right)-\{0\}\right),
$$


with coordinates $\left(v,\left(z_{1}, \ldots, z_{m-1}\right), z_{m} \neq 0\right)$, it is

$$
p r_{1}^{*} L \otimes p r_{2}^{*} \mathrm{FT}_{\psi}\left(K_{m-1}\right) \otimes p r_{3}^{*} \mathscr{L}_{\chi}[1] \otimes \mathscr{L}_{\psi(\operatorname{Tr}(-f(v) z))}(-n m) \text {. }
$$

Now consider the object

on

$$
\mathscr{N}:=p r_{1}^{*} L \otimes p r_{2}^{*} \mathrm{FT}_{\psi}\left(K_{m-1}\right) \otimes p r_{3}^{*} \overline{\mathbb{Q}}_{l}[1] \otimes \mathscr{L}_{\psi(\operatorname{Tr}(-f(v) z))}(-n m)
$$

$$
\mathscr{R}\left[1 / z_{m}\right]:=V \times \operatorname{Lin} \operatorname{Maps}\left(\mathbb{A}^{m-1}, \mathbb{A}^{1}\right) \times\left(\operatorname{Lin} \operatorname{Maps}\left(\mathbb{A}^{1}, \mathbb{A}^{1}\right)-\{0\}\right) .
$$

This object is visibly perverse irreducible on $\mathscr{R}\left[1 / z_{m}\right]$, and it is related to $\mathrm{FT}_{\psi}(M(\chi))$ by

$$
\mathrm{FT}_{\psi}(M(\chi))=\mathscr{N} \otimes \mathscr{L}_{\chi\left(z_{m}\right)} .
$$

Now denote by $\mathscr{U}$ the open set $\operatorname{Aff}^{\vee}\left[1 / z_{m}\right]$ of the entire space

$$
\operatorname{Aff} \mathbb{V}^{\vee}:=\operatorname{Lin} \operatorname{Maps}\left(\mathbb{A}^{m}, \mathbb{A}^{n}\right) \times \operatorname{Lin} \operatorname{Maps}\left(\mathbb{A}^{m}, \mathbb{A}^{1}\right),
$$

with coordinates $(W, z)$, where $z_{m}$ is invertible, and by

$$
i: \mathscr{R}\left[1 / z_{m}\right] \rightarrow \operatorname{Aff}^{\vee}\left[1 / z_{m}\right]
$$

the inclusion, a closed immersion. Then $i_{*} \mathcal{N}$ on $\operatorname{Aff}^{\vee}\left[1 / z_{m}\right]$ is still perverse irreducible on $\operatorname{Aff}^{\vee}\left[1 / z_{m}\right]$, and we still have the relation

$$
\mathrm{FT}_{\psi}(M(\chi))=i_{*} \mathscr{N} \otimes \mathscr{L}_{\chi\left(z_{m}\right)} \quad \text { on } \operatorname{Aff}^{\vee}\left[1 / z_{m}\right]
$$

Since we know that $\mathrm{FT}_{\psi}(M(\chi))$ on $\mathrm{Aff}^{\vee}$ is supported in $\mathrm{Aff}^{\vee}\left[1 / z_{m}\right]$, if we denote by

$$
k: \operatorname{Aff}^{\vee}\left[1 / z_{m}\right] \rightarrow \text { Aff }^{\vee}
$$

the inclusion, we have

$$
\mathrm{FT}_{\psi}(M(\chi))=k_{!}\left(i_{*} \mathscr{N} \otimes \mathscr{L}_{\chi\left(z_{m}\right)}\right) \text { on } \mathrm{Aff}^{\vee} .
$$

Since $k$ is affine, both $k_{!}\left(i_{*} \mathscr{N} \otimes \mathscr{L}_{\chi\left(z_{m}\right)}\right)$ and $R k_{*}\left(i_{*} \mathscr{N} \otimes \mathscr{L}_{\chi\left(z_{m}\right)}\right)$ are perverse, and the middle extension $k_{! *}\left(i_{*} \mathscr{N} \otimes \mathscr{L}_{\chi\left(z_{m}\right)}^{m}\right)$ is

$$
\text { Image }\left(k_{!}\left(i_{*} \mathscr{N} \otimes \mathscr{L}_{\chi\left(z_{m}\right)}\right) \rightarrow R k_{*}\left(i_{*} \mathscr{N} \otimes \mathscr{L}_{\chi\left(z_{m}\right)}\right)\right) \text {. }
$$

By [KaLa, 6.5.2], applied to the object $i_{*} \mathscr{N}$ on

$$
\operatorname{Aff}{ }^{\vee}\left[1 / z_{m}\right]=\operatorname{Lin} \operatorname{Maps}\left(\mathbb{A}^{m}, \mathbb{A}^{n}\right) \times \operatorname{Lin} \operatorname{Maps}\left(\mathbb{A}^{m-1}, \mathbb{A}^{1}\right) \times \mathbb{G}_{m},
$$

with coordinates $\left(W,\left(z_{1}, \ldots, z_{m-1}\right), z_{m} \neq 0\right)$, there exists an integer $N_{0}$, which depends only on $i_{*} \mathscr{N}$, such that for any character $\chi$ of order $d>N_{0}$, the canonical "forget supports" map is an isomorphism

$$
k_{!}\left(i_{*} \mathscr{N} \otimes \mathscr{L}_{\chi\left(z_{m}\right)}\right) \cong R k_{*}\left(i_{*} \mathscr{N} \otimes \mathscr{L}_{\chi\left(z_{m}\right)}\right)
$$

But whenever this map is an isomorphism, we have

$$
\mathrm{FT}_{\psi}(M(\chi))=k_{!}\left(i_{*} \mathscr{N} \otimes \mathscr{L}_{\chi\left(z_{m}\right)}\right)=k_{! *}\left(i_{*} \mathscr{N} \otimes \mathscr{L}_{\chi\left(z_{m}\right)}\right) .
$$


As $i_{*} \mathscr{N} \otimes \mathscr{L}_{\chi\left(z_{m}\right)}$ is perverse irreducible on $\operatorname{Aff}^{\vee}\left[1 / z_{m}\right], k_{! *}\left(i_{*} \mathscr{N} \otimes \mathscr{L}_{\chi\left(z_{m}\right)}\right)$ is perverse irreducible on $\mathrm{Aff}^{\vee}$. Thus $\mathrm{FT}_{\psi}(M(\chi))$ is perverse irreducible on $\mathrm{Aff}^{\vee}$ for all $\chi$ of order $>N_{0}$.

To prove (3), we will show that for $\chi$ of order $>N_{0}$, we have

$$
R\left(p r_{2}\right)_{!}\left(p r_{1}^{*} L \otimes f_{\mathrm{aff}}^{*} K(\chi)\right)[n m] \approx R\left(p r_{2}\right)_{*}\left(p r_{1}^{*} L \otimes f_{\mathrm{aff}}^{*} K(\chi)\right)[n m] .
$$

By duality, we have

$$
R\left(p r_{2}\right)_{*}\left(p r_{1}^{*} L \otimes f_{\text {aff }}^{*} K(\chi)\right)[n m] \approx D\left(R\left(p r_{2}\right)_{!}\left(p r_{1}^{*} D L \otimes f_{\text {aff }}^{*} D K(\chi)\right)[n m](n m)\right),
$$

so it is the same to show that the natural pairing makes

$$
R\left(p r_{2}\right)_{!}\left(p r_{1}^{*} L \otimes f_{\mathrm{aff}}^{*} K(\chi)\right)[n m]
$$

and

$$
R\left(p r_{2}\right)_{!}\left(p r_{1}^{*} D L \otimes f_{\mathrm{aff}}^{*} D K(\chi)\right)[n m](n m)
$$

dual to each other.

Now we interpret this on the Fourier Transform side, using the compatibility of $r$-dimensional FT with duality [KaLa, 2.1.5]:

$$
D\left(\mathrm{FT}_{\psi}(N) \cong \mathrm{FT}_{\tilde{\psi}}(D N)(r)\right. \text {. }
$$

We must show that for $\chi$ of order $>N_{0}$, the two objects

$$
\begin{aligned}
& \mathrm{FT}_{\psi} R\left(p r_{2}\right)_{!}\left(p r_{1}^{*} L \otimes f_{\mathrm{aff}}^{*} K(\chi)\right)[n m], \\
& \mathrm{FT}_{\bar{\psi}} R\left(p r_{2}\right)_{!}\left(p r_{1}^{*} D L \otimes f_{\mathrm{aff}}^{*} D K(\chi)\right)[n m](2 n m+m)
\end{aligned}
$$

are dual. The first we have shown above to be

$$
k_{!}\left(i_{*} \mathscr{N} \otimes \mathscr{L}_{\chi\left(z_{m}\right)}\right)
$$

with

$$
\mathscr{N}:=p r_{1}^{*} L \otimes p r_{2}^{*} \mathrm{FT}_{\psi}\left(K_{m-1}\right) \otimes p r_{3}^{*} \overline{\mathbb{Q}}_{l}[1] \otimes \mathscr{L}_{\psi(\operatorname{Tr}(-f(v) z))}(-n m)
$$

The second is, by the same calculation using $\mathrm{FT}_{\bar{\psi}}$,

$$
k_{!}\left(i_{*} \mathscr{M} \otimes \mathscr{L}_{\bar{\chi}\left(z_{m}\right)}\right) \text {, }
$$

with

$$
\mathscr{M}:=p r_{1}^{*} D L \otimes p r_{2}^{*} \mathrm{FT}_{\bar{\psi}}\left(D K_{m-1}\right) \otimes p r_{3}^{*} \overline{\mathbb{Q}}_{l}[1] \otimes \mathscr{L}_{\bar{\psi}(\operatorname{Tr}(-f(v) z))}(n m+m)=D \mathscr{N} .
$$

Thus we are reduced to showing that for $\chi$ of order $>N_{0}$,

$$
k_{!}\left(i_{*} \mathscr{N} \otimes \mathscr{L}_{\chi\left(z_{m}\right)}\right) \quad \text { and } \quad k_{!}\left(i_{*} D \mathscr{N} \otimes \mathscr{L}_{\bar{\chi}\left(z_{m}\right)}\right)
$$

are dual. We may rewrite the dual of the latter term as

$$
D k_{!}\left(i_{*} D \mathscr{N} \otimes \mathscr{L}_{\bar{\chi}\left(z_{m}\right)}\right)=R k_{*}\left(i_{*} \mathscr{N} \otimes \mathscr{L}_{\chi\left(z_{m}\right)}\right),
$$

so it is the same to show that for $\chi$ of order $>N_{0}$,

$$
k_{!}\left(i_{*} \mathscr{N} \otimes \mathscr{L}_{\chi\left(z_{m}\right)}\right) \cong R k_{*}\left(i_{*} \mathscr{N} \otimes \mathscr{L}_{\chi\left(z_{m}\right)}\right),
$$


which is once again [KaLa, 6.5.2]. This proves (3). Notice that we in fact show that for each nontrivial $\chi$, the "forget supports" map

$$
R\left(p r_{2}\right)_{!}\left(p r_{1}^{*} L \otimes f_{\mathrm{aff}}^{*} K(\chi)\right)[n m] \rightarrow R\left(p r_{2}\right)_{*}\left(p r_{1}^{*} L \otimes f_{\mathrm{aff}}^{*} K(\chi)\right)[n m]
$$

is an isomorphism if and only if the "forget supports" map

$$
k_{!}\left(i_{*} \mathscr{N} \otimes \mathscr{L}_{\chi\left(z_{m}\right)}\right) \rightarrow R k_{*}\left(i_{*} \mathscr{N} \otimes \mathscr{L}_{\chi\left(z_{m}\right)}\right)
$$

is an isomorphism. In proving (2), we showed that if

$$
k_{!}\left(i_{*} \mathscr{N} \otimes \mathscr{L}_{\chi\left(z_{m}\right)}\right) \rightarrow R k_{*}\left(i_{*} \mathscr{N} \otimes \mathscr{L}_{\chi\left(z_{m}\right)}\right)
$$

is an isomorphism, then $R\left(p r_{2}\right)_{!}\left(p r_{1}^{*} L \otimes f_{\text {aff }}^{*} K(\chi)\right)[n m]$ is perverse irreducible. this proves (4). Q.E.D.

As a special case of the above result, we obtain the following corollaries. The $\mathscr{D}$-module analogue of Corollary 2.4.3 was conjectured by Dwork [Dw, 15.4 via 11.1.3].

2.4.2. Corollary. Suppose we are given:

$k$, an algebraically closed field of characteristic $p \neq l$;

$V$, an affine smooth irreducible $k$-scheme of finite type;

$f: V \rightarrow \mathbb{A}^{m}$, an arbitrary morphism;

$\pi: V \rightarrow \mathbb{A}^{n}$, a closed immersion;

$m-1$ multiplicative characters $\chi_{1}, \ldots, \chi_{m-1}$ of a finite subfield of $k$.

For $\chi$ any (possibly trivial) multiplicative character of a finite subfield of $k$, consider the Kummer sheaf $\mathscr{L}_{\chi}$ on $\mathbb{G}_{m}$ and its extension by direct image $j_{*} \mathscr{L}_{\chi}$ to $\mathbb{A}^{1}$. For any multiplicative character $\chi_{m}$, denote by $K\left(\chi_{1}, \chi_{2}, \ldots, \chi_{m}\right)$ the external tensor product

$$
K(\chi):=\bigotimes_{i=1, \ldots, m} p r_{i}^{*} j_{*} \mathscr{L}_{\chi_{i}}[1] \text { on } \mathbb{A}^{m}=\mathbb{A}^{1} \times \cdots \times \mathbb{A}^{1}
$$

Then:

(1) $K\left(\chi_{1}, \chi_{2}, \ldots, \chi_{m}\right)$ is perverse irreducible on $\mathbb{A}^{m}$, and $H_{c}\left(\mathbb{A}^{m}, K(\chi)\right)=$ 0 if some $\chi_{i}$ is nontrivial.

(2) There exists an integer $N_{0}$, depending only on the data

$$
\left(k, V, f, \pi, \chi_{1}, \chi_{2}, \ldots, \chi_{m-1}\right) \text {, }
$$

such that if the order of $\chi_{m}$ is $>N_{0}$, then the perverse sheaf

$$
R\left(p r_{2}\right)_{!}\left(f_{\mathrm{aff}}^{*} K\left(\chi_{1}, \chi_{2}, \ldots, \chi_{m}\right)\right)[n m+\operatorname{dim} V]
$$

on $\operatorname{Aff} \operatorname{Maps}\left(\mathbb{A}^{n}, \mathbb{A}^{m}\right)$ is irreducible.

Proof. This is the special case when $L$ Is $\overline{\mathbb{Q}}_{l, V}[\operatorname{dim} V]$, which is perverse irreducible on $V$ because $V$ is smooth and irreducible, and when $K_{m-1}$ is $K\left(\chi_{1}, \chi_{2}, \ldots, \chi_{m-1}\right)$. Q.E.D.

\subsubsection{Corollary. Suppose we are given:}

$k$, an algebraically closed field of characteristic $p \neq l$; 
$f: \mathbb{A}^{n} \rightarrow \mathbb{A}^{m}$, an arbitrary morphism;

$n$ multiplicative characters $\rho_{1}, \ldots, \rho_{n}$ of a finite subfield of $k$; and

$m-1$ multiplicative characters $\chi_{1}, \ldots, \chi_{m-1}$ of a finite subfield of $k$.

Using the notation of 2.4.2, for any multiplicative character $\chi_{m}$, denote by $K\left(\chi_{1}, \chi_{2}, \ldots, \chi_{m}\right)$ the external tensor product

$$
K(\chi):=\bigotimes_{i=1, \ldots, m} p r_{i}^{*} j_{*} \mathscr{L}_{\chi_{i}}[1] \text { on } \mathbb{A}^{m}=\mathbb{A}^{1} \times \cdots \times \mathbb{A}^{1}
$$

and denote by $L\left(\rho_{1}, \ldots, \rho_{n}\right)$ the external tensor product

$$
L(\rho):=\bigotimes_{i=1, \ldots, n} p r_{i}^{*} j_{*} \mathscr{L}_{\rho_{i}}[1] \text { on } \mathbb{A}^{n}=\mathbb{A}^{1} \times \cdots \times \mathbb{A}^{1} .
$$

Then:

(1) $K\left(\chi_{1}, \chi_{2}, \ldots, \chi_{m}\right)$ is perverse irreducible on $\mathbb{A}^{m}$, and $H_{c}\left(\mathbb{A}^{m}, K(\chi)\right)=$ 0 if some $\chi_{i}$ is nontrivial.

(2) $L\left(\rho_{1}, \ldots, \rho_{n}\right)$ is perverse irreducible on $\mathbb{A}^{n}$, and $H_{c}\left(\mathbb{A}^{n}, L(\rho)\right)=0$ if some $\rho_{i}$ is nontrivial.

(3) There exists an integer $N_{0}$, depending only on the data

$$
\left(k, f, \rho_{1}, \ldots, \rho_{n}, \chi_{1}, \chi_{2}, \ldots, \chi_{m-1}\right),
$$

such that if the order of $\chi_{m}$ is $>N_{0}$, then the perverse sheaf

$$
R\left(p r_{2}\right)_{!}\left(p r_{1}^{*} L\left(\rho_{1}, \ldots, \rho_{n}\right) \otimes f_{\mathrm{aff}}^{*} K\left(\chi_{1}, \chi_{2}, \ldots, \chi_{m}\right)\right)[n m+\operatorname{dim} V]
$$

on $\operatorname{Aff} \operatorname{Maps}\left(\mathbb{A}^{n}, \mathbb{A}^{m}\right)$ is irreducible.

Proof. This is the special case of 2.4 .1 when $V$ is $\mathbb{A}^{n}, \pi$ is the identity map, $L$ is $L\left(\rho_{1}, \ldots, \rho_{n}\right)$, and $K_{m-1}$ is $K\left(\chi_{1}, \chi_{2}, \ldots, \chi_{m-1}\right)$. Q.E.D.

\subsection{Uniformity for the integer $N_{0}$ in Theorem 2.4.1(3).}

2.5.1. Uniformity Theorem. Suppose we are given:

$S=\operatorname{Spec}(A)$, with $A \subset \mathbb{C}$ a subring of $\mathbb{C}$ which is normal, and finitely generated over $\mathbb{Z}$

$V$ an affine $S$-scheme of finite type;

$f: V \rightarrow\left(\mathbb{A}^{m}\right)_{S}$, an arbitrary $S$-morphism;

$\pi: V \rightarrow\left(\mathbb{A}^{n}\right)_{S}$, a closed $S$-immersion;

$K_{m-1}$, an object of $D_{c}^{b}\left(\left(\mathbb{A}^{m-1}\right)_{S}, \overline{\mathbb{Q}}_{l}\right)$ such that for each geometric point $s$ of $S, K_{m-1} \mid\left(\mathbb{A}^{m-1}\right)_{s}$ is perverse irreducible on $\left(\mathbb{A}^{m-1}\right)_{s}$;

$L$, an object of $D_{c}^{b}\left(V, \overline{\mathbb{Q}}_{l}\right)$ such that for each geometric point $s$ of $S$, $K_{m-1} \mid V_{s}$ is perverse irreducible on $V_{s}$.

For each geometric point $s$ of $S$ of characteristic $p>0$, apply Theorem 2.4.1 to the fibre over $s$ of this data and denote by $N_{0}(s) \geq 1$ the least integer that "works" in conclusion (3). Then there exists an integer $N \geq 1$, a dense open set 
$U$ of $S[1 / N]$, and an integer $N_{1} \geq 1$ such that for every geometric point $u$ of $U, N_{0}(u) \leq N_{1}$.

Proof. This is essentially an exercise in applying the uniformity results of [KaLa, §3], the terminology and results of which we will use freely. Fix a stratification $\mathscr{V}$ of $V$ to which $L$ is adapted and a stratification $\mathscr{A}$ of $\left(\mathbb{A}^{m-1}\right)_{S}$ to which $K_{m-1}$ is adapted.

For each integer $d \geq 1$, denote by $\varphi_{d}(t)$ in $\mathbb{Z}[x]$ the $d$ th cyclotomic polynomial and by $\mathbb{Z}\left[1 / d, \zeta_{d}\right]$ the ring $\mathbb{Z}[1 / d, t] /\left(\varphi_{d}(t)\right)$. The sheaf $\mathscr{L}_{\chi}$, for $\chi$ of order $d$, can be constructed universally on $\left(\mathbb{G}_{m}\right)_{\mathbb{Z}\left[1 / d, \zeta_{d}\right]}$, and its extension by zero $j_{1} \mathscr{L}_{\chi}$ to $\mathbb{A}^{1}$ lives on $\left(\mathbb{A}^{1}\right)_{\mathbb{Z}}\left[1 / d, \zeta_{d}\right]$. A key point in what follows is that for every $d$ and for every $\chi$ of order $d, j_{1} \mathscr{L}_{\chi}$ is always adapted to the same stratification $\left(\mathbb{G}_{m},\{0\}\right)$ of $\mathbb{A}^{1}$.

In terms of the stratifications $\mathscr{V}$ and $\mathscr{A}$, and the map $f_{\text {aff }}$, one constructs

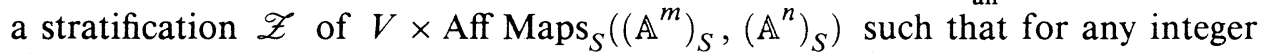
$d \geq 1$ and any character $\chi$ of order $d$, after the base change from $S$ to $S \otimes_{\mathbb{Z}} \mathbb{Z}\left[1 / d, \zeta_{d}\right]$, the object $p r_{1}^{*} L \otimes f_{\text {aff }}^{*} K(\chi)$ is adapted to $\mathscr{Z}$.

We will need to apply [KaLa, 3.3.3] to this stratification $\mathscr{Z}$ and to the morphism

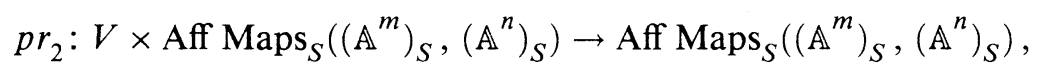

viewed as a map of $S$-schemes. By [KaLa, 3.3.3] there exists an integer $N \geq 1$, a dense open set $U$ in $S[1 / N\}$ and a stratification $\mathscr{B}$ of

$$
\text { Aff } \operatorname{Maps}_{U}\left(\left(\mathbb{A}^{m}\right)_{U},\left(\mathbb{A}^{n}\right)_{U}\right) \text {, }
$$

with the following property: For any integer $d \geq 1$ and any character $\chi$ of order $d$, after the base change from $U$ to $U \otimes_{\mathbb{Z}} \mathbb{Z}\left[1 / d, \zeta_{d}\right]$, each of the objects

$$
R\left(p r_{2}\right)_{!}\left(p r_{1}^{*} L \otimes f_{\mathrm{aff}}^{*} K(\chi)\right)[n m], \quad R\left(p r_{2}\right)_{*}\left(p r_{1}^{*} L \otimes f_{\mathrm{aff}}^{*} K(\chi)\right)[n m],
$$

is adapted to $\mathscr{B}$, and its formation commutes with arbitrary change of base on $U \otimes_{\mathbb{Z}} \mathbb{Z}\left[1 / d, \zeta_{d}\right]$.

At the expense of further shrinking $U$, we may further assume that for each strat $B_{\alpha}$ of $B$, as $U$-scheme, say $f_{\alpha}: B_{\alpha} \rightarrow U$, all the sheaves $R^{i}\left(f_{\alpha}\right)_{!}\left(\mathbb{F}_{l}\right)$ on $U$ are lisse.

This is a property of $f_{\alpha}$, which is stable by arbitrary base change on $U$. If it holds, then after any base change $U^{\prime} \rightarrow U$ with $U^{\prime}$ connected, every geometric fibre of $f_{\alpha}$ meets every connected component of $B_{\alpha} \times_{U} U^{\prime}$. (Indeed, we reduce by base change to treating the case when $U$ is connected. Then for each nonempty connected component $C$ of $B_{\alpha}$, with structural morphism $f_{\alpha, C}$, the cohomology $\bigoplus_{i} R^{i}\left(f_{\alpha, C}\right)_{!}(\mathbb{Z} / l \mathbb{Z})$ is itself lisse, being a direct factor of $\bigoplus_{i} R^{i}\left(f_{\alpha}\right)_{!}(\mathbb{Z} / l \mathbb{Z})$. Now as $C$ is nonempty, at least one of the geometric fibres of $f_{\alpha, C}$ is nonempty, so has some dimension $d \geq 0$, and, therefore, by proper base change $R^{2 d}\left(f_{\alpha, C}\right)_{!}(\mathbb{Z} / l \mathbb{Z})$ is nonzero. Therefore $\bigoplus_{i} R^{i}\left(f_{\alpha, C}\right)_{!}(\mathbb{Z} / l \mathbb{Z})$ is both lisse and nonzero. As $U$ is connected, this sheaf is everywhere nonzero, so again by proper base change the map $f_{\alpha, C}$ must be surjective.) 
2.5.2. Lemma. Let $U$ be a normal $\mathbb{Z}[1 / l]$-scheme of finite type, $X / U$ a $U$ scheme of finite type, and $\mathscr{X}$ a stratification of $X$ such that each strata $X_{\alpha}$ as $U$-scheme, say $f_{\alpha}: X_{\alpha} \rightarrow U$, has all the sheaves $R^{i}\left(f_{\alpha}\right)_{!}\left(\mathbb{F}_{l}\right)$ on $U$ lisse.

(1) Let $M$ be an object of $D_{c}^{b}\left(X, \overline{\mathbb{Q}}_{l}\right)$, which is adapted to the stratification $\mathscr{X}$. For every geometric point $u$ in $U$, we denote by $M_{u}:=M \mid X_{u}$ the induced object of $D_{c}^{b}\left(X_{u}, \overline{\mathbb{Q}}_{l}\right)$. Then the following conditions are equivalent.

(a) $M=0$ in $D_{c}^{b}\left(X, \overline{\mathbb{Q}}_{l}\right)$.

(b) For every geometric point $u$ of $U, M_{u}=0$ in $D_{c}^{b}\left(X_{u}, \overline{\mathbb{Q}}_{l}\right)$.

(c) In every connected component of $U$ there exists a geometric point $u$ of $U$ for which $M_{u}=0$ in $D_{c}^{b}\left(X_{u}, \overline{\mathbb{Q}}_{l}\right)$.

(2) Let $M$ and $N$ be two objects in $D_{c}^{b}\left(X, \overline{\mathbb{Q}}_{l}\right)$, both of which are adapted to the stratification $\mathscr{X}$, and let $\varphi: M \rightarrow N$ be a morphism. For every geometric point $u$ in $U$, we denote by $\varphi_{u}: M_{u} \rightarrow N_{u}$ the induced morphism on fibres. Then the following conditions are equivalent.

(a) $\varphi$ is an isomorphism in $D_{c}^{b}\left(X, \overline{\mathbb{Q}}_{l}\right)$.

(b) For every geometric point $u$ of $U, \varphi_{u}$ is an isomorphism in $D_{c}^{b}\left(X_{u}, \overline{\mathbb{Q}}_{l}\right)$.

(c) In every connected component of $U$ there exists a geometric point $u$ of $U$ for which $\varphi_{u}$ is an isomorphism in $D_{c}^{b}\left(X_{u}, \overline{\mathbb{Q}}_{l}\right)$.

Proof. Making the base change from $U$ to each of its connected components, we may reduce to the case when $U$ is connected. For each strat $X_{\alpha}$, a lisse sheaf $\mathscr{F}_{\alpha}$ on $X_{\alpha}$ vanishes if and only if it vanishes at some point of each connected component of $X_{\alpha}$; but if $U$ is connected, any geometric fibre of $X_{\alpha} \rightarrow U$ meets every connected component of $X_{\alpha}$. Apply this to each cohomology sheaf $\mathscr{H}^{i}(M)$, restricted to each $X_{\alpha}$.

(2) follows by applying (1) to the mapping cylinder of $\varphi$. Q.E.D.

Let us return now to an integer $d \geq 2$ and the universal $\mathscr{L}_{\chi}$ for a character $\chi$ of order $d$. After the base change from $U$ to $U \otimes_{\mathbb{Z}} \mathbb{Z}\left[1 / d, \zeta_{d}\right]$, consider the "forget supports" map

$$
R\left(p r_{2}\right)_{!}\left(p r_{1}^{*} L \otimes f_{\mathrm{aff}}^{*} K(\chi)\right)[n m] \rightarrow R\left(p r_{2}\right)_{*}\left(p r_{1}^{*} L \otimes f_{\mathrm{aff}}^{*} K(\chi)\right)[n m] .
$$

Both source and target are of formation compatible with arbitrary base change on $U \otimes_{\mathbb{Z}} \mathbb{Z}\left[1 / d, \zeta_{d}\right]$. Because $U[1 / d]$ is connected, every connected component of $U \otimes_{\mathbb{Z}} \mathbb{Z}\left[1 / d, \zeta_{d}\right]$ maps onto $U[1 / d]$. So by Lemma 2.5.2, for each integer $d \geq 2$ the following four conditions are equivalent.

(1d) The "forget supports" maps is an isomorphism

$$
R\left(p r_{2}\right)_{!}\left(p r_{1}^{*} L \otimes f_{\text {aff }}^{*} K(\chi)\right)[n m] \approx R\left(p r_{2}\right)_{*}\left(p r_{1}^{*} L \otimes f_{\text {aff }}^{*} K(\chi)\right)[n m]
$$

on Aff Maps $\otimes U \otimes_{\mathbb{Z}} \mathbb{Z}\left[1 / d, \zeta_{d}\right]$.

(2d) For every geometric point $u$ of $U[1 / d]$ and every character $\chi$ of order $d$, the fibre over $u$ of the above "forget supports" map is an isomorphism.

(3d) There exists a geometric point $u$ of $U[1 / d]$ and a character $\chi$ of order $d$, such that the fibre over $u$ of above "forget supports" map is an isomorphism. 
(4d) Over the geometric generic point " $\mathbb{C}$ " of $U$, corresponding to the given inclusion $A \subset \mathbb{C}$, the "forget supports" map is an isomorphism

$$
R\left(p r_{2}\right)_{!}\left(p r_{1}^{*} L \otimes f_{\mathrm{aff}}^{*} K(\chi)\right)[n m] \approx R\left(p r_{2}\right)_{*}\left(p r_{1}^{*} L \otimes f_{\mathrm{aff}}^{*} K(\chi)\right)[n m]
$$

on Aff Maps $\mathbb{C}_{\mathbb{C}}$ for some character $\chi$ of order $d$.

In view of the equivalence of $(2 \mathrm{~d})$ with $(4 \mathrm{~d})$, in order to prove Theorem 2.5.1 it suffices to show the existence of $N_{1}$ such that for any character $\chi$ of order $d \geq N_{1}$, condition (4d) holds, i.e., the "forget supports" map is an isomorphism

$$
R\left(p r_{2}\right)_{!}\left(p r_{1}^{*} L \otimes f_{\mathrm{aff}}^{*} K(\chi)\right)[n m] \approx R\left(p r_{2}\right)_{*}\left(p r_{1}^{*} L \otimes f_{\mathrm{aff}}^{*} K(\chi)\right)[n m]
$$

on Aff Maps $_{\mathbb{C}}$.

All that we know right now is that for any geometric point $u$ in $U$ whose residue characteristic $p_{u}$ is positive, the equivalent condition ( $3 \mathrm{~d}$ ) holds for all integers $d \geq N_{0}(u)$, which are prime to $p_{u}$. This already shows that $(4 \mathrm{~d})$ holds for an infinity of $d$ 's. We will deduce from the fact that (4d) holds for an infinity of $d$ 's that (4d) must hold for all sufficiently large $d$ 's.

In order to do this, factor the morphism

$$
p r_{2}: V \times \text { Aff Maps } \rightarrow \text { Aff Maps }
$$

as

$$
\begin{array}{r}
V \times \text { Aff Maps } \\
\downarrow f_{\text {aff }} \times \text { id } \\
\mathbb{A}^{m} \times \text { Aff Maps } \\
\quad p_{m} \times \text { id } \\
\mathbb{A}^{1} \times \text { Aff Maps } \\
\downarrow \pi_{2}\left(:=p r_{2}\right)
\end{array}
$$

Aff Maps.

We denote the composition of the first two maps simply by

$$
\varphi: V \times \text { Aff Maps } \rightarrow \mathbb{A}^{1} \times \text { Aff Maps } .
$$

Consider also the composite morphism

$$
\begin{gathered}
V \times \text { Aff Maps } \\
\downarrow_{\text {id } \times f_{\text {aff }}} \\
V \times \mathbb{A}^{m} \\
\downarrow^{\text {id } \times p r_{1,2, \ldots, m-1}} \\
V \times \mathbb{A}^{m-1}
\end{gathered}
$$

which we denote simply as

$$
\gamma: V \times \text { Aff Maps } \rightarrow V \times \mathbb{A}^{m-1} .
$$


The object $p r_{1}^{*} L \otimes f_{\text {aff }}^{*} K(\chi)$ on $V \times$ Aff Maps is the tensor product

$$
\gamma^{*}\left(L \otimes K_{m-1}\right) \otimes \varphi^{*}\left(j \mathscr{L}_{\chi}[1] \otimes \overline{\mathbb{Q}}_{l, \text { Aff Maps }}\right) .
$$

We define two complexes $M_{!}$and $M_{*}$ on $\mathbb{A}^{1} \times$ Aff Maps as follows:

$$
\begin{aligned}
& M_{!}:=R \varphi_{!}\left(\gamma^{*}\left(L \otimes K_{m-1}\right)\right), \\
& M_{*}:=R \varphi_{*}\left(\gamma^{*}\left(L \otimes K_{m-1}\right)\right) .
\end{aligned}
$$

These objects are independent of $\chi$, and there is a natural "forget supports" $\operatorname{map} M_{!} \rightarrow M_{*}$.

By the projection formula, we have

$$
\begin{aligned}
& R\left(p r_{2}\right)_{!}\left(p r_{1}^{*} L \otimes f_{\mathrm{aff}}^{*} K(\chi)\right) \\
& \quad=R\left(\pi_{2}\right)_{!}\left(M_{!} \otimes j_{!} \mathscr{L}_{\chi}[1]\right)=R\left(\pi_{2}\right)_{!}\left(j_{!}\left(j^{*} M_{!} \otimes \mathscr{L}_{\chi}\right)\right)[1] \\
& R\left(p r_{2}\right)_{*}\left(p r_{1}^{*} L \otimes f_{\mathrm{aff}}^{*} K(\chi)\right) \\
& \quad=R\left(\pi_{2}\right)_{*}\left(M_{*} \otimes j_{!} \mathscr{L}_{\chi}[1]\right)=R\left(\pi_{2}\right)_{*}\left(j_{!}\left(j^{*} M_{*} \otimes \mathscr{L}_{\chi}\right)\right)[1] .
\end{aligned}
$$

We must show that the natural forget supports map

$$
R\left(\pi_{2}\right)_{!}\left(j_{!}\left(j^{*} M_{!} \otimes \mathscr{L}_{\chi}\right)\right) \rightarrow R\left(\pi_{2}\right)_{*}\left(j_{!}\left(j^{*} M_{*} \otimes \mathscr{L}_{\chi}\right)\right)
$$

is an isomorphism on Aff Maps $_{\mathbb{C}}$ for all $\chi$ of sufficiently high order, given that it is an isomorphism for an infinity of $\chi$.

Let us denote by

$$
\begin{gathered}
\bar{j}: \mathbb{G}_{m} \times \text { Aff Maps } \rightarrow \mathbb{P}^{1} \times \text { Aff Maps }, \\
k: \mathbb{A}^{1} \times \text { Aff Maps } \rightarrow \mathbb{P}^{1} \times \text { Aff Maps },
\end{gathered}
$$

the inclusion, and by

$$
\bar{\pi}_{2}: \mathbb{P}^{1} \times \text { Aff Maps } \rightarrow \text { Aff Maps, }
$$

the second projection. In terms of $\bar{j}$ and $\bar{\pi}_{2}$, we have the tautological formulas

$$
\begin{aligned}
R\left(\pi_{2}\right)_{!}\left(j_{!}\left(j^{*} M_{!} \otimes \mathscr{L}_{\chi}\right)\right) & =R\left(\bar{\pi}_{2}\right)_{*}\left(\bar{j}_{!}\left(j^{*} M_{!} \otimes \mathscr{L}_{\chi}\right)\right), \\
R\left(\pi_{2}\right)_{*}\left(j_{!}\left(j^{*} M_{*} \otimes \mathscr{L}_{\chi}\right)\right) & =R\left(\bar{\pi}_{2}\right)_{*} R k_{*}\left(j_{!}\left(j^{*} M_{*} \otimes \mathscr{L}_{\chi}\right)\right) .
\end{aligned}
$$

So we may rewrite the "forget supports" map as

$$
R\left(\bar{\pi}_{2}\right)_{*}\left(\bar{j}_{!}\left(j^{*} M_{!} \otimes \mathscr{L}_{\chi}\right)\right) \rightarrow R\left(\bar{\pi}_{2}\right)_{*} R k_{*}\left(j_{!}\left(j^{*} M_{*} \otimes \mathscr{L}_{\chi}\right)\right)
$$

As proven in [KaLa, 6.5], for any $N$ on $\mathbb{G}_{m} \times$ Aff Maps, the maps

$$
\bar{j}_{!}\left(N \otimes \mathscr{L}_{\chi}\right) \rightarrow R k_{*} j_{!}\left(N \otimes \mathscr{L}_{\chi}\right) \rightarrow R \bar{j}_{*}\left(N \otimes \mathscr{L}_{\chi}\right)
$$

are isomorphisms for all $\chi$ of sufficiently high order. Applying this to each of the objects $j^{*} M_{\text {! }}$ and $j^{*} M_{*}$, we see that for all $\chi$ of sufficiently high order, we may rewrite the "forget supports" map as

$$
\left.\left.R\left(\bar{\pi}_{2}\right)_{*}\left(\bar{j}_{!}\left(j^{*} M_{!}\right) \otimes \bar{j}_{!} \mathscr{L}_{\chi}\right)\right) \rightarrow R\left(\bar{\pi}_{2}\right)_{*}\left(\bar{j}_{!}\left(j^{*} M_{*}\right) \otimes \bar{j}_{!} \mathscr{L}_{\chi}\right)\right) .
$$


In terms of the second projection

$$
p_{2}: \mathbb{G}_{m} \times \text { Aff Maps } \rightarrow \text { Aff Maps },
$$

this is the map

$$
R p_{2 !}\left(j^{*} M_{!} \otimes \mathscr{L}_{\chi}\right) \rightarrow R p_{2 !}\left(j^{*} M_{*} \otimes \mathscr{L}_{\chi}\right) .
$$

This map is an isomorphism for $\chi$ if and only if the mapping cylinder

$$
N:=\left[j^{*} M_{!} \rightarrow j^{*} M_{*}\right]
$$

has the property

$$
R p_{2 !}\left(N \otimes \mathscr{L}_{\chi}\right)=0
$$

We know that this holds for an infinity of $\chi$, and we have only to apply the following lemma, taking $X:=$ Aff Maps $_{\mathbb{C}}$.

2.5.3. Lemma. Let $X$ be a scheme of finite type over an algebraically closed field $k$ of characteristic $\neq l$ and $N$ an object of $D_{c}^{b}\left(\mathbb{G}_{m} \times X, \overline{\mathbb{Q}}_{l}\right)$. Suppose for an infinity of multiplicative characters $\chi$ of finite order prime to char $(k)$, $R\left(p_{2}\right)_{!}\left(N \otimes \mathscr{L}_{\chi}\right)=0$. Then all but finitely many characters $\chi$ of finite order prime to $\operatorname{char}(k), R\left(p_{2}\right)_{!}\left(N \otimes \mathscr{L}_{\chi}\right)=0$.

Proof. By definition of $D_{c}^{b}\left(\mathbb{G}_{m} \times X, \overline{\mathbb{Q}}_{l}\right)$, there exists a finite extension $E_{\lambda}$ of $\mathbb{Q}_{l}$ such that $N$ is the extension of scalars of an object $N_{\lambda}$ of $D_{c}^{b}\left(\mathbb{G}_{m} \times X, E_{\lambda}\right)$. There are finitely many nonzero cohomology sheaves $\mathscr{H}^{i}\left(N_{\lambda}\right)$, each of which, being constructible, has all its stalks of dimension bounded by some constant $C$. Inside $\overline{\mathbb{Q}}_{l}$, there are only finitely many extensions of $E_{\lambda}$ of degree $\leq C$, so all such extensions are contained in some finite extension $F_{\lambda}$ of $E_{\lambda}$. The group of all roots of unity in $F_{\lambda}$ is finite, say of order $D$. We claim that for any character $\chi$ of finite order prime to $\operatorname{char}(k)$, whose order does not divide $D$, we have $R\left(p_{2}\right)_{!}\left(N \otimes \mathscr{L}_{\chi}\right)=0$ on $X$.

By proper base change, we may reduce to the case when $X$ is a point, i.e., the spectrum of an algebraically closed field $K$ of characteristic $\operatorname{char}(k)$. Then $N$ in $D_{c}^{b}\left(\mathbb{G}_{m, K}, E_{\lambda}\right)$ satisfies

$$
H_{c}\left(\mathbb{G}_{m, K}, N \otimes \mathscr{L}_{\chi}\right)=0
$$

for an infinity of $\chi$. Consider the spectral sequence

$$
E_{2}^{p, q}=H_{c}^{p}\left(\mathbb{G}_{m, K}, \mathscr{H}^{q}(N) \otimes \mathscr{L}_{\chi}\right) \Rightarrow H_{c}^{p+q}\left(\mathbb{G}_{m, K}, N \otimes \mathscr{L}_{\chi}\right) .
$$

The $E_{2}$ terms vanish except possibly for $p=0,1$, or 2 . So the only possibly nonvanishing differential is $d_{2}^{0, q}: E_{2}^{0, q} \rightarrow E_{2}^{2, q-1} ;$ but $E_{2}^{2, q-1}$ is $H_{c}^{2}\left(\mathbb{G}_{m, K}, \mathscr{H}^{q-1}(N) \otimes \mathscr{L}_{\chi}\right)$, which vanishes for $\chi$ of sufficiently high order. For if $U$ is a dense open set of $\mathbb{G}_{m, K}$ on which $\mathscr{H}^{q-1}(N)$ is lisse, this $H_{c}^{2}$ is nonzero if and only if $\mathscr{L}_{\bar{\chi}} \mid U$ is a quotient of $\mathscr{H}^{q-1}(N) \mid U$, and by JordanHölder theory there are only finitely many possible irreducible quotients. (In fact, this $H_{c}^{2}$ vanishes for $\chi$ of order not dividing $D$, as one sees by looking 
at the local monodromy at either 0 or $\infty$.) Since $H_{c}\left(\mathbb{G}_{m, K}, N \otimes \mathscr{L}_{\chi}\right)=0$ for an infinity of $\chi$ 's, there exists an infinity of $\chi$ 's for which both

$$
H_{c}\left(\mathbb{G}_{m, K}, N \otimes \mathscr{L}_{\chi}\right)=0
$$

and

$$
E_{2}^{2, q-1}=0 \text { for all } q
$$

For any such $\chi$, the spectral sequence degenerates at $E_{2}$. Consequently, for an infinity of $\chi$ 's we have

$$
H_{c}\left(\mathbb{G}_{m, K}, \mathscr{H}^{q}(N) \otimes \mathscr{L}_{\chi}\right)=0 \text { for all } q .
$$

In other words, each of the cohomology sheaves $\mathscr{H}^{q}(N)$ is a constructible $E_{\lambda}$-sheaf $\mathscr{F}$, which itself satisfies the condition

$$
H_{c}\left(\mathbb{G}_{m, K}, \mathscr{F} \otimes \mathscr{L}_{\chi}\right)=0 \text { for an infinity of } \chi \text { 's. }
$$

Fix one such $\chi$. The vanishing of $H_{c}^{0}$ shows that $\mathscr{F} \otimes \mathscr{L}_{\chi}$ has no punctual sections. The vanishing of $\chi_{c}\left(\mathbb{G}_{m, K}, \mathscr{F} \otimes \mathscr{L}_{\chi}\right)$ gives, by the Euler-Poincaré formula on $\mathbb{G}_{m, K}$,

$$
\begin{aligned}
0 & =\chi_{c}\left(\mathbb{G}_{m, K}, \mathscr{F} \otimes \mathscr{L}_{\chi}\right) \\
& =-\sum_{x \text { in } \mathbb{G}_{m, K}} \text { total } \operatorname{drop}_{x}\left(\mathscr{F} \otimes \mathscr{L}_{\chi}\right)-\operatorname{Swan}_{0}\left(\mathscr{F} \otimes \mathscr{L}_{\chi}\right)-\operatorname{Swan}_{\infty}\left(\mathscr{F} \otimes \mathscr{L}_{\chi}\right) .
\end{aligned}
$$

Because $\mathscr{F} \otimes \mathscr{L}_{\chi}$ has no punctual sections, all the terms on the right side are negative, so they all must vanish. Their vanishing means that $\mathscr{F} \otimes \mathscr{L}_{\chi}$ is lisse on $\mathbb{G}_{m, K}$ and tame at both 0 and $\infty$. Therefore $\mathscr{F}$ itself is a successive extension of sheaves $\mathscr{L}_{\Lambda}$, thanks to the known structure of the tame $\pi_{1}$ of $\mathbb{G}_{m, K}$, for various characters $\Lambda$, not necessarily of finite order, of this tame $\pi_{1}$. Because $\mathscr{F}$ has rank bounded by $C$ and is an $E_{\lambda}$-sheaf, all the characters $\chi$ of finite order such that $\mathscr{L}_{\chi}$ occurs in $\mathscr{F}$ as a Jordan-Hölder constituent have order dividing $D$; therefore, for any character $\rho$ of finite order prime to $\operatorname{char}(K)$ whose order does not divide $D, \mathscr{F} \otimes \mathscr{L}_{\rho}$ is a successive extension of sheaves $\mathscr{L}_{\Lambda}$ with $\Lambda$ nontrivial and, hence, has $H_{c}\left(\mathbb{G}_{m, K}, \mathscr{F} \otimes \mathscr{L}_{\rho}\right)=0$. Q.E.D.

This concludes (!) the proof of the Uniformity Theorem 2.5.1. Q.E.D.

2.5.4. Corollary. Hypotheses and notation are as in the Uniformity Theorem 2.5.1.

(1) For all $\chi$ of finite order $>1$, the objects

$$
R\left(p r_{2}\right)_{!}\left(p r_{1}^{*} L \otimes f_{\mathrm{aff}}^{*} K(\chi)\right)[n m] \mid \text { Aff }^{\mathrm{Maps}} \mathbb{C}_{\mathbb{C}}
$$

and

$$
R\left(p r_{2}\right)_{*}\left(p r_{1}^{*} L \otimes f_{\mathrm{aff}}^{*} K(\chi)\right)[n m] \mid \mathrm{Aff}, \mathrm{Maps}_{\mathbb{C}}
$$

are perverse on Aff Maps $_{\mathbb{C}}$.

(2) For any $\chi$ of finite order $>N_{1}$, the object

$$
R\left(p r_{2}\right)_{!}\left(p r_{1}^{*} L \otimes f_{\text {aff }}^{*} K(\chi)\right)[n m] \mid \text { Aff } \text { Maps }_{\mathbb{C}}
$$

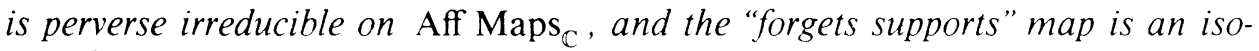
morphism. 
Proof. For any character $\chi$ of order $d>1$ (resp. $>N_{1}$ ) conclusion (1) (resp. (2)) holds at all geometric points of $U[1 / d]$ of finite characteristic. The passage to the geometric generic point $\mathbb{C}$ of $U$ results from [BBD, 6.1.9]. Q.E.D.

2.5.5. Remark. See [GKZ, Theorem 2.11] for a $\mathscr{D}$-module analogue of 2.5 .4 over $\mathbb{C}$.

\section{Applications to Bertini AND LefsChetZ-STYle theorems FOR HYPERSURFACE SECTIONS}

3.0. In this section, we will obtain theorems of Bertini and Lefschetz type as a consequence of what the general theory gives in the case when $m=1, K$ is the perverse sheaf $K$ on $\mathbb{A}^{1}$ given by

$$
K:=R j_{*} \overline{\mathbb{Q}}_{l}[1] \text { for } j: \mathbb{G}_{m} \rightarrow \mathbb{A}^{1} \text { the inclusion, }
$$

and variable data $(V, f, \pi, L)$. The basic observation that we need to get started is

3.0.1. Lemma. Over an algebraically closed field $k$ of characteristic $p \neq l$, the perverse sheaf $K$ on $\mathbb{A}^{1}$, given by

$$
K:=R j_{*} \overline{\mathbb{Q}}_{l}[1] \text { for } j: \mathbb{G}_{m} \rightarrow \mathbb{A}^{1} \text { the inclusion, }
$$

has $H_{c}\left(\mathbb{A}^{1}, K\right)=0$.

Proof. By the inversion $x \mapsto 1 / x$ on $\mathbb{P}^{1}, H_{c}\left(\mathbb{A}^{1}, K\right)$ becomes the ordinary cohomology $H\left(\mathbb{A}^{1}, j ! \overline{\mathbb{Q}}_{l}[1]\right)$, so we are reduced to showing that $H^{i}\left(\mathbb{A}^{1}, j ! \overline{\mathbb{Q}}_{l}\right)=0$ for $i=0,1,2$. By Grothendieck's Euler-Poincaré Formula, we have $\chi\left(\mathbb{A}^{1}, j ! \overline{\mathbb{Q}}_{l}\right)$ $=0$. For cohomological dimension reasons, we have $H^{i}\left(\mathbb{A}^{1}, j \overline{\mathbb{Q}}_{l}\right)=0$ for $i>1$. The group $H^{0}\left(\mathbb{A}^{1}, j \overline{\mathbb{Q}}_{l}\right)$ vanishes because $j ! \overline{\mathbb{Q}}_{l}$ has no punctual sections and its stalk at one point (the origin) vanishes; therefore, the remaining group $H^{1}\left(\mathbb{A}^{1}, j_{1} \overline{\mathbb{Q}}_{l}\right)$ vanishes, since $\chi\left(\mathbb{A}^{1}, j_{!} \overline{\mathbb{Q}}_{l}\right)=0$.

An alternate proof is to notice that $R \Gamma_{c}\left(\mathbb{A}^{1}, K\right)[1]$ is the stalk at zero of the Fourier Transform $\mathrm{FT}_{\psi}(K)$ and then to appeal to the result [Ka4, A2] that

$$
\mathrm{FT}_{\psi}\left(R j_{*} \overline{\mathbb{Q}}_{l}[1]\right) \cong j_{!} \overline{\mathbb{Q}}_{l}[1] \text {. Q.E.D. }
$$

Applying Main Theorem 1.5 to this case, we get

3.0.2. Corollary. Suppose we are given:

$k$, an algebraically field of characteristic $p \neq l$;

$V$, a separated $k$-scheme of finite type;

$f: V \rightarrow \mathbb{A}^{1}$, an arbitrary function;

$\pi: V \rightarrow \mathbb{A}^{n}$, a quasi-finite morphism.

Then for any perverse $L$ on $V$, the object

$$
M(L):=R\left(p r_{2}\right)_{!}\left(p r_{1}^{*} L \otimes f_{\mathrm{aff}}^{*} R j_{*} \overline{\mathbb{Q}}_{l}[1]\right)[n]
$$

on $\operatorname{Aff} \operatorname{Maps}\left(\mathbb{A}^{n}, \mathbb{A}^{1}\right)$ is semiperverse. 
3.1. What does this mean concretely? The semiperversity on $\operatorname{Aff} \operatorname{Maps}\left(\mathbb{A}^{n}, \mathbb{A}^{1}\right)$ of $M(L)$, together with its constructibility, implies that there exists a dense open set $\mathscr{U}$ of $\operatorname{Aff} \operatorname{Maps}\left(\mathbb{A}^{n}, \mathbb{A}^{1}\right)$ over which

(1) all the cohomology sheaves $\mathscr{H}^{i} M(L)$ are lisse on $\mathscr{U}$,

(2) $\mathscr{H}^{i} M(L)$ vanishes on $\mathscr{U}$ for $i>-\operatorname{dim} A f f \operatorname{Maps}\left(\mathbb{A}^{n}, \mathbb{A}^{1}\right)=-n-1$.

3.2. By duality, it follows that over $\mathscr{U}$, the dual

$$
D M(L)=R\left(p r_{2}\right)_{*}\left(p r_{1}^{*} D L \otimes f_{\text {aff }}^{*} j_{!} \overline{\mathbb{Q}}_{l}[1](1)\right)[n](n)
$$

has lisse cohomology sheaves on $\mathscr{U}$, whose formation commutes with arbitrary change of base on $\mathscr{U}$ and whose cohomology sheaves vanish for $i<-n-1$.

3.3. For each point $(A, b)$ in $\operatorname{Aff} \operatorname{Maps}\left(\mathbb{A}^{n}, \mathbb{A}^{1}\right)$ corresponding to the affine map $x \mapsto A x+b$, let us denote by $f_{A, b}: V \rightarrow \mathbb{A}^{1}$ the function $v \mapsto f(v)+$ $A \pi(v)+b$, by $j_{A, b}: V\left[1 / f_{A, b}\right] \rightarrow V$ the open inclusion, and by

$$
i_{A, b}: V \cap\left(f_{A, b}=0\right) \rightarrow V
$$

the closed inclusion. Then if $(A, b)$ lies in $\mathscr{U}$, the stalk at $(A, b)$ of

$$
R\left(p r_{2}\right)_{*}\left(p r_{1}^{*} D L \otimes f_{\mathrm{aff}}^{*} j_{!} \overline{\mathbb{Q}}_{l}[1](1)\right)[n](n)
$$

is

$R \Gamma\left(V, D L \otimes\left(j_{A, b}\right)_{!} \overline{\mathbb{Q}}_{l}\right)[n+1](n+1)=R \Gamma\left(V,\left(j_{A, b}\right)_{!}\left(j_{A, b}\right)^{*} D L\right)[n+1](n+1)$.

The $i$ th cohomology group of this complex is

$$
H^{i+n+1}\left(V,\left(j_{A, b}\right)_{!}\left(j_{A, b}\right)^{*} D L\right)(n+1),
$$

and for $(A, b)$ in $\mathscr{U}$, it vanishes for $i<-n-1$. In other words,

$$
H^{a}\left(V,\left(j_{A, b}\right)_{!}\left(j_{A, b}\right)^{*} D L\right)=0 \text { if } a<0 \text { and if }(A, b) \text { in } \mathscr{U} .
$$

Now take the long exact cohomology sequence of the short exact sequence

$$
0 \rightarrow\left(j_{A, b}\right)_{!}\left(j_{A, b}\right)^{*} D L \rightarrow D L \rightarrow\left(i_{A, b}\right)_{*}\left(i_{A, b}\right)^{*} D L \rightarrow 0 .
$$

We find that if $(A, b)$ in $\mathscr{U}$, the restriction map

$$
H^{a}(V, D L) \rightarrow H^{a}\left(V \cap\left(f_{A, b}=0\right),\left(i_{A, b}\right)^{*} D L\right)
$$

is an isomorphism for $a<-1$ and injective for $a=-1$.

Exchanging the roles of $L$ and $D L$, we get

3.4. Theorem. Suppose we are given:

$k$, an algebraically closed field of characteristic $p \neq l$;

$V$, a separated $k$-scheme of finite type;

$f: V \rightarrow \mathbb{A}^{1}$, an arbitrary function;

$\pi: V \rightarrow \mathbb{A}^{n}$, a quasi-finite morphism. 
Then for any perverse $L$ on $V$, there exists a dense open set $\mathscr{U}$ of $\operatorname{Aff} \operatorname{Maps}\left(\mathbb{A}^{n}, \mathbb{A}^{1}\right)$ such that if $(A, b)$ in $\mathscr{U}$ then

(1) $H^{a}\left(V,\left(j_{A, b}\right)_{!}\left(j_{A, b}\right)^{*} L\right)=0$ for $a<0$,

(2) the restriction map

$$
H^{a}(V, L) \rightarrow H^{a}\left(V \cap\left(f_{A, b}=0\right),\left(i_{A, b}\right)^{*} L\right)
$$

is an isomorphism for $a<-1$ and injective for $a=-1$.

3.4.1. Corollary ("Weak Lefschetz"). Suppose we are given:

$k$, an algebraically closed field of characteristic $p \neq l$;

$V$, a separated $k$-scheme of finite type, which is a local complete intersection, purely of dimension $d>0$;

$f: V \rightarrow \mathbb{A}^{1}$, an arbitrary function;

$\pi: V \rightarrow \mathbb{A}^{n}$, a quasi-finite morphism.

Then for any lisse $\overline{\mathbb{Q}}_{l}$-sheaf $\mathscr{F}$ on $V$, there exists a dense open set $\mathscr{U}$ of $\operatorname{Aff} \operatorname{Maps}\left(\mathbb{A}^{n}, \mathbb{A}^{1}\right)$ such that if $(A, b)$ in $\mathscr{U}$ then

(1) $H^{a}\left(V,\left(j_{A, b}\right)_{!}\left(j_{A, b}\right)^{*} \mathscr{F}\right)=0$ for $a<d$,

(2) the restriction map

$$
H^{a}(V, \mathscr{F}) \rightarrow H^{a}\left(V \cap\left(f_{A, b}=0\right),\left(i_{A, b}\right)^{*} \mathscr{F}\right)
$$

is an isomorphism for $a<d-1$ and injective for $a=d-1$.

Proof. This is simply Theorem 3.4, applied to the perverse sheaf $L=\mathscr{F}[d]$ on $V(\mathscr{F}[d]$ is perverse on $V$ because $V$ is a local complete intersection, everywhere of dimension $d$ ). Q.E.D.

\subsubsection{Corollary ("Weak Bertini"). Suppose we are given:}

$k$, an algebraically closed field of characteristic $p \neq l$;

$V$, a separated $k$-scheme of finite type, which is a connected local complete intersection, purely of dimension $d>1$;

$f: V \rightarrow \mathbb{A}^{1}$, an arbitrary function;

$\pi: V \rightarrow \mathbb{A}^{n}$, a quasi-finite morphism.

Then for any finite étale covering $\rho: E \rightarrow V$ with $E$ connected, there exists a dense open set $\mathscr{U}$ of $\operatorname{Aff} \operatorname{Maps}\left(\mathbb{A}^{n}, \mathbb{A}^{1}\right)$ such that if $(A, b)$ in $\mathscr{U}$ then the pullback $\rho^{-1}\left(V \cap\left(f_{A, b}=0\right)\right)$ of $E$ to $V \cap\left(f_{A, b}=0\right)$

$$
\begin{array}{r}
\rho^{-1}\left(V \cap\left(f_{A, b}=0\right)\right) \subset E \\
\downarrow \\
V
\end{array}
$$

is connected.

Proof. Corollary 3.4.1 applied to the constant sheaf $\overline{\mathbb{Q}}_{l}$ on $V$ and $a=0$ shows that there exists an open dense $\mathscr{U}$ in Aff such that for $(A, b)$ in $\mathscr{U}$, we have $H^{0}\left(V, \overline{\mathbb{Q}}_{l}\right) \approx H^{0}\left(V \cap\left(f_{A, b}=0\right), \overline{\mathbb{Q}}_{l}\right)$, i.e., $V \cap\left(f_{A, b}=0\right)$ is connected. Now apply this same result to the data $(k, E, f \circ \rho, \pi \circ \rho)$. Q.E.D. 
3.4.3. Corollary ("Weak Smooth Bertini"). Suppose we are given:

$k$, an algebraically closed field of characteristic $p \neq l$;

$V$, an irreducible smooth separated $k$-scheme of finite type of dimension $d>1$

$f: V \rightarrow \mathbb{A}^{1}$, an arbitrary function;

$\pi: V \rightarrow \mathbb{A}^{n}$, an unramified morphism.

Then:

(1) There exists a dense open set $\mathscr{U}$ of $\operatorname{Aff} \operatorname{Maps}\left(\mathbb{A}^{n}, \mathbb{A}^{1}\right)$ such that if $(A, b)$ in $\mathscr{U}$ then $V \cap\left(f_{A, b}=0\right)$ is smooth and irreducible.

(2) For any finite étale covering $\rho: E \rightarrow V$ with $E$ irreducible, there exists a dense open set $\mathscr{U}$ of $\operatorname{Aff} \operatorname{Maps}\left(\mathbb{A}^{n}, \mathbb{A}^{1}\right)$ such that if $(A, b)$ in $\mathscr{U}$ then the pullback $\rho^{-1}\left(V \cap\left(f_{A, b}=0\right)\right)$ of $E$ to $V \cap\left(f_{A, b}=0\right)$

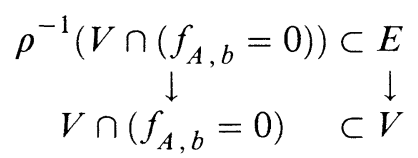

is smooth and irreducible.

Proof. Just as in Corollary 3.4.2, statement (2) is just statement (1) for the data $(k, E, f \circ \rho, \pi \circ \rho)$. To prove (1), it suffices to show there exists a dense open set $\mathscr{U}_{1}$ of $\operatorname{Aff} \operatorname{Maps}\left(\mathbb{A}^{n}, \mathbb{A}^{1}\right)$ such that if $(A, b)$ in $\mathscr{U}_{1}$ then $V \cap\left(f_{A, b}=0\right)$ is smooth. For we already know there exists a dense open set $\mathscr{U}$ of $\operatorname{Aff} \operatorname{Maps}\left(\mathbb{A}^{n}, \mathbb{A}^{1}\right)$ such that if $(A, b)$ in $\mathscr{U}$ then $V \cap\left(f_{A, b}=0\right)$ is connected; so for $(A, b)$ in $\mathscr{U} \cap \mathscr{U}_{1}$, we find that $V \cap\left(f_{A, b}=0\right)$ is smooth and connected, hence, irreducible. Q.E.D.

The smoothness amounts to the following lemma, which is certainly well known but for which I do not know a reference.

3.5. Lemma. Suppose we are given:

$k$, an algebraically closed field;

$V$, an irreducible smooth separated $k$-scheme of finite type of dimension $d \geq 1$

$f: V \rightarrow \mathbb{A}^{1}$, an arbitrary function;

$\pi: V \rightarrow \mathbb{A}^{n}$, an unramified morphism, given explicitly by $n$ functions $x_{1}, \ldots, x_{n}$ on $V$ whose differentials $d x_{i} \operatorname{span} \Omega_{V / k}^{1}$ at every point of $V$.

Then there exists a dense open set $\mathscr{U}$ of

$$
\begin{aligned}
\operatorname{Aff} \operatorname{Maps}\left(\mathbb{A}^{n}, \mathbb{A}^{1}\right) & =\operatorname{Lin} \operatorname{Maps}\left(\mathbb{A}^{n}, \mathbb{A}^{1}\right) \times \mathbb{A}^{1} \\
& =\mathbb{A}^{1} \times \operatorname{Lin} \operatorname{Maps}\left(\mathbb{A}^{n}, \mathbb{A}^{1}\right)
\end{aligned}
$$

over which the map

$$
\begin{aligned}
& F: V \times \operatorname{Lin} \operatorname{Maps}\left(\mathbb{A}^{n}, \mathbb{A}^{1}\right) \rightarrow \mathbb{A}^{1} \times \operatorname{Lin} \operatorname{Maps}\left(\mathbb{A}^{n}, \mathbb{A}^{1}\right), \\
& \left(v,\left(a_{i}\right)\right) \mapsto\left(f(v)+\sum_{i=1, \ldots, n} a_{i} x_{i}(v),\left(a_{i}\right)\right) .
\end{aligned}
$$


is smooth, of relative dimension $d-1$. In particular, if $(A, b)$ in $\mathscr{U}$, then $V \cap\left(f_{A,-b}=0\right)$ is smooth of dimension $d-1$.

Proof. On $V$, consider the rank $d$ vector bundle $\Omega_{V / k}^{1}$, the rank $n$ trivial bundle $\mathscr{O}^{n}$ and the surjective homomorphism of vector bundles $\mathscr{O}^{n} \rightarrow \Omega_{V / k}^{1}$ given by the $n$ sections $d x_{1}, \ldots, d x_{n}$. We denote by $\mathscr{K}$ the kernel of this map:

$$
0 \rightarrow \mathscr{K} \rightarrow \mathscr{O}^{n} \rightarrow \Omega_{V / k}^{1} \rightarrow 0 .
$$

Thus $\mathscr{K}$ is a vector bundle over $V$ of rank $n-d$.

On the product $V \times \operatorname{Lin} \operatorname{Maps}\left(\mathbb{A}^{n}, \mathbb{A}^{1}\right)$, with coordinates $\left(v,\left(a_{i}\right)\right)$, consider the morphism

$$
\begin{aligned}
& F: V \times \operatorname{Lin} \operatorname{Maps}\left(\mathbb{A}^{n}, \mathbb{A}^{1}\right) \rightarrow \mathbb{A}^{1} \times \operatorname{Lin} \operatorname{Maps}\left(\mathbb{A}^{n}, \mathbb{A}^{1}\right), \\
& \left(v,\left(a_{i}\right)\right) \mapsto\left(f(v)+\sum_{i=1, \ldots, n} a_{i} x_{i}(v),\left(a_{i}\right)\right) .
\end{aligned}
$$

In the product $V \times \operatorname{Lin} \operatorname{Maps}\left(\mathbb{A}^{n}, \mathbb{A}^{1}\right)$, consider the subvariety Crit Pt consisting of the critical points of $F$, i.e., of the points $\left(v,\left(a_{i}\right)\right)$ where

$$
d f=-\sum_{i=1, \ldots, n} a_{i} d x_{i} \quad \text { in } \Omega_{V / k}^{1} \otimes k(v) .
$$

Since the $d x_{i} \operatorname{span} \Omega_{V / k}^{1}$ at every point, we see that the first projection $p r_{1}$ : Crit $\mathrm{Pt} \rightarrow V$ is a smooth map of relative dimension $n-d$; indeed, it is a torsor under $\mathscr{K}$. Therefore Crit $\mathrm{Pt}$ is smooth, of dimension $n$.

Now consider the restriction of $F$ to CritPt:

$$
F \mid \text { Crit Pt: Crit Pt } \rightarrow \mathbb{A}^{1} \times \operatorname{Lin} \operatorname{Maps}\left(\mathbb{A}^{n}, \mathbb{A}^{1}\right) \text {. }
$$

This is a morphism of separated schemes of finite type over $k$ and so is a separated morphism of finite type. Its source, Crit Pt, is lower dimensional than its target $\mathbb{A}^{1} \times \operatorname{Lin} \operatorname{Maps}\left(\mathbb{A}^{n}, \mathbb{A}^{1}\right)$. We apply part (2) of the following lemma.

3.6. Lemma. Suppose we are given:

$k$, an algebraically closed field of characteristic $\neq l$;

$S$, a normal irreducible separated $k$-scheme of finite type;

$p: X \rightarrow S$, a separated morphism of finite type.

Then:

(1) If $\operatorname{dim}(X) \leq \operatorname{dim}(S)$, there exists an open dense set $\mathscr{U}$ in $S$ and an integer $N$, such that over $\mathscr{U}$ the morphism $p$ has finite fibres, each with precisely $N$ geometric points.

(2) If $\operatorname{dim}(X)<\operatorname{dim}(S)$ then $N=0$, i.e., there exists an open dense set $\mathscr{U}$ in $S$ over which $X_{\mathscr{U}}$ is empty.

Proof. Replacing $S$ by a dense open set $\mathscr{U}$ of $S$ does not change $\operatorname{dim}(S)$ or $\operatorname{dim}(X)$ and allows us to assume

all the sheaves $R^{i} p_{1}(\mathbb{Z} / I \mathbb{Z})$ are lisse on $S$. 
Further replacing $S$ by a finite étale surjective covering of itself allows us to assume that

$$
\text { all the sheaves } R^{i} p_{!}(\mathbb{Z} / l \mathbb{Z}) \text { are constant on } S \text {. }
$$

We will show that under $(* *)$, the map $p$ has finite fibres.

If $p$ has empty fibres, i.e., if $X$ is empty, there is nothing to prove.

If $X$ is nonempty, let us denote by $D \geq 0$ the maximum of the dimensions of the fibres of $p$. By proper base change and the fact that for $Y$ a separated $r$-dimensional scheme of finite type over an algebraically closed field of characteristic $\neq l$, we have

$$
H_{c}^{i}(Y, \mathbb{Z} / l \mathbb{Z})=0 \text { for } i>2 r,
$$

$\operatorname{dim} H_{c}^{2 r}(Y, \mathbb{Z} / l \mathbb{Z})=$ number of irreducible components of dimension $r$,

we see that

$$
2 D=\text { maximum integer } N \text { such that } R^{N} p_{!}(\mathbb{Z} / l \mathbb{Z}) \neq 0 .
$$

Since $R^{2 D} p_{!}(Z / l \mathbb{Z})$ is constant on $S$, say with value $A_{2 D}$, denoting by $d:=$ $\operatorname{dim}(S)$ the Leray spectral sequence for $R p$, shows

$$
\begin{aligned}
H_{c}^{i}(X, \mathbb{Z} / l \mathbb{Z}) & =0 \text { for } i>2 d+2 D, \\
H_{c}^{2 d+2 D}(X, \mathbb{Z} / l \mathbb{Z}) & =H_{c}^{2 d}\left(S, R^{2 D} p_{!}(\mathbb{Z} / l \mathbb{Z})\right) \\
& =H_{c}^{2 d}(S, \mathbb{Z} / l \mathbb{Z}) \otimes A_{2 D} \neq 0 .
\end{aligned}
$$

Therefore we find that $\operatorname{dim}(X)=D+d=\operatorname{dim}(S)+D$. Since $D \geq 0$ and $\operatorname{dim}(X) \leq \operatorname{dim}(S)$, we conclude that $D=0$ and that $\operatorname{dim}(X)=\operatorname{dim}(S)$. The integer $\bar{N}$ is the rank of the sheaf $p_{!}(\mathbb{Z} / l \mathbb{Z}) \mid \mathscr{U}$. Q.E.D.

Now let us return to the morphism

$$
F \mid \text { Crit Pt: Crit Pt } \rightarrow \mathbb{A}^{1} \times \operatorname{Lin} \operatorname{Maps}\left(\mathbb{A}^{n}, \mathbb{A}^{1}\right) .
$$

By part (2) of Lemma 3.6, there exists a dense open set $\mathscr{U}$ in

$$
\mathbb{A}^{1} \times \operatorname{Lin} \operatorname{Maps}\left(\mathbb{A}^{n}, \mathbb{A}^{1}\right)
$$

over which Crit Pt is empty. This means precisely that over $\mathscr{U}$, the morphism

$$
\begin{aligned}
& F: V \times \operatorname{Lin} \operatorname{Maps}\left(\mathbb{A}^{n}, \mathbb{A}^{1}\right) \rightarrow \mathbb{A}^{1} \times \operatorname{Lin} \operatorname{Maps}\left(\mathbb{A}^{n}, \mathbb{A}^{1}\right), \\
& \left(v,\left(a_{i}\right)\right) \mapsto\left(f(v)+\sum_{i=1, \ldots, n} a_{i} x_{i}(v),\left(a_{i}\right)\right) .
\end{aligned}
$$

is smooth, of relative dimension $d-1$.

Thus if $(A, b)=\left(\left(a_{i}\right), b\right)$ lies in $\mathscr{U}$, the fibre over it of $F$ is smooth over $k$; but this fibre is $V \cap\left(f_{A}=b\right)$, i.e., it is $V \cap\left(f_{A,-b}=0\right)$. Q.E.D.

Here is a relative version of the above lemma. 
3.7. Lemma. Suppose we are given:

$k$, an algebraically closed field;

$T$, an irreducible smooth separated $k$-scheme of finite type;

$P: V \rightarrow T$, a smooth separated morphism of finite type, everywhere of relative dimension $d>0$;

$f: V \rightarrow \mathbb{A}^{1}$, an arbitrary function;

$\pi: V \rightarrow \mathbb{A}^{n} \times T$, an unramified T-morphism, given explicitly by $n$ functions $x_{1}, \ldots, x_{n}$ on $V$ whose differentials $d x_{i} \operatorname{span} \Omega_{V / T}^{1}$ at every point of $V$.

Then there exists a dense open set $\mathscr{U}$ of

$$
\begin{aligned}
\operatorname{Aff} \operatorname{Maps}\left(\mathbb{A}^{n}, \mathbb{A}^{1}\right) \times T & =\operatorname{Lin} \operatorname{Maps}\left(\mathbb{A}^{n}, \mathbb{A}^{1}\right) \times \mathbb{A}^{1} \times T \\
& =\mathbb{A}^{1} \times \operatorname{Lin} \operatorname{Maps}\left(\mathbb{A}^{n}, \mathbb{A}^{1}\right) \times T
\end{aligned}
$$

over which the morphism

$$
\begin{aligned}
V \times & \operatorname{Lin} \operatorname{Maps}\left(\mathbb{A}^{n}, \mathbb{A}^{1}\right) \\
& \quad \mid(v, A) \mapsto(f(v)+A \pi(v), A, P(v)) \\
\mathbb{A}^{1} \times & \operatorname{Lin} \operatorname{Maps}\left(\mathbb{A}^{n}, \mathbb{A}^{1}\right) \times T
\end{aligned}
$$

is smooth, everywhere of relative dimension $d-1$.

Proof. The proof proceeds exactly as above, with $\operatorname{Lin} \operatorname{Maps}\left(\mathbb{A}^{n}, \mathbb{A}^{1}\right)$ replaced systematically by $\operatorname{Lin} \operatorname{Maps}\left(\mathbb{A}^{n}, \mathbb{A}^{1}\right) \times T$. Q.E.D.

This relative version gives, by an obvious induction on $m$, the following:

3.8. Lemma. Suppose we are given:

an integer $m \geq 1$;

$k$, an algebraically closed field;

$T$, an irreducible smooth separated $k$-scheme of finite type;

$P: V \rightarrow T$, a smooth separated morphism of finite type, everywhere of relative dimension $d \geq m$;

$f: V \rightarrow \mathbb{A}^{m}$, an arbitrary morphism; and

$\pi: V \rightarrow \mathbb{A}^{n} \times T$, an unramified T-morphism, given explicitly by $n$ functions $x_{1}, \ldots, x_{n}$ on $V$ whose differentials $d x_{i} \operatorname{span} \Omega_{V / T}^{1}$ at every point of $V$.

Then there exists a dense open set $\mathscr{U}$ of

$$
\begin{aligned}
\operatorname{Aff} \operatorname{Maps}\left(\mathbb{A}^{n}, \mathbb{A}^{m}\right) \times T & =\operatorname{Lin} \operatorname{Maps}\left(\mathbb{A}^{n}, \mathbb{A}^{m}\right) \times \mathbb{A}^{m} \times T \\
& =\mathbb{A}^{m} \times \operatorname{Lin} \operatorname{Maps}\left(A^{n}, \mathbb{A}^{m}\right) \times T
\end{aligned}
$$

over which the morphism

$$
\begin{aligned}
& V \times \operatorname{Lin} \operatorname{Maps}\left(\mathbb{A}^{n}, \mathbb{A}^{m}\right) \\
& \quad \mid(v, A) \mapsto(f(v)+A \pi(v), A, P(v)) \\
& \mathbb{A}^{m} \times \operatorname{Lin} \operatorname{Maps}\left(\mathbb{A}^{n}, \mathbb{A}^{m}\right) \times T
\end{aligned}
$$

is smooth, everywhere of relative dimension $d-m$.

Taking $T=\operatorname{Spec}(k)$ gives 
3.8.1. Corollary. Suppose we are given:

an integer $m \geq 1$;

$k$, an algebraically closed field;

$V$, a smooth separated $k$-scheme of finite type, everywhere of relative dimension $d \geq m$;

$f: V \rightarrow \mathbb{A}^{m}$, an arbitrary morphism; and

$\pi: V \rightarrow \mathbb{A}^{n}$, an unramified morphism, given explicitly by $n$ functions $x_{1}, \ldots, x_{n}$ on $V$ whose differentials $d x_{i} \operatorname{span} \Omega_{V / k}^{1}$ at every point of $V$.

Then there exists a dense open set $\mathscr{U}$ of

$$
\begin{aligned}
\operatorname{Aff} \operatorname{Maps}\left(\mathbb{A}^{n}, \mathbb{A}^{m}\right) & =\operatorname{Lin} \operatorname{Maps}\left(\mathbb{A}^{n}, \mathbb{A}^{m}\right) \times \mathbb{A}^{m} \\
& =\mathbb{A}^{m} \times \operatorname{Lin} \operatorname{Maps}\left(\mathbb{A}^{n}, \mathbb{A}^{m}\right)
\end{aligned}
$$

such that if $(A, b)$ lies in $\mathscr{U}$ then $V \cap(f(v)+A \pi(v)=b)$ is smooth, everywhere of dimension $d-m$.

3.9. Variants for several simultaneous hypersurface sections. Using the above result, we can give variants of our Bertini and Lefschetz style results. The idea now is to apply the main theorem in the general case to the semiperverse sheaf $K$ on $\mathbb{A}^{m}$ defined as

$$
K:=R j_{*} \overline{\mathbb{Q}}_{l}[2 m-1] \text { for } j: \mathbb{A}^{m}-\{0\} \rightarrow \mathbb{A}^{m}, \text { the inclusion. }
$$

3.9.1. Lemma. Over an algebraically closed field $k$ of characteristic $p \neq l$, the object $K$ on $\mathbb{A}^{m}, m \geq 1$, given by

$$
K:=R j_{*} \overline{\mathbb{Q}}_{l}[2 m-1] \text { for } j: \mathbb{A}^{m}-\{0\} \rightarrow \mathbb{A}^{m} \text { the inclusion, }
$$

has $H_{c}\left(\mathbb{A}^{m}, K\right)=0$.

Proof. Notice that $R \Gamma_{c}\left(\mathbb{A}^{m}, K\right)[1]$ is the stalk at zero of the Fourier Transform $\mathrm{FT}_{\psi}(K)$, and then appeal to the result $[\mathrm{Ka} 4, \mathrm{~A} 4]$

$$
\mathrm{FT}_{\psi}\left(R j_{*} \overline{\mathbb{Q}}_{l}[2 m-1]\right) \cong j_{!} \overline{\mathbb{Q}}_{l}[1] \text {. Q.E.D. }
$$

\subsubsection{Lemma. For}

$$
K:=R j_{*} \overline{\mathbb{Q}}_{l}[2 m-1] \text { for } j: \mathbb{A}^{m}-\{0\} \rightarrow \mathbb{A}^{m} \text { the inclusion, }
$$

its dual $D K$ is given by $D K=j_{!} \overline{\mathbb{Q}}_{l}[1](m)$.

Proof. Duality interchanges $R j_{*}$ and $R j_{!}$and the dual of $\overline{\mathbb{Q}}_{l}[2 m-1]$ is $\overline{\mathbb{Q}}_{l}[1](m)$.

Q.E.D.

3.9.3. Lemma. The cohomology groups $H^{i}\left(\mathbb{A}^{m}, j ! \overline{\mathbb{Q}}_{l}\right)=0$ for all $i$.

Proof. This is the (shifted) dual of the vanishing of $H_{c}\left(\mathbb{A}^{m}, K\right)$. Q.E.D.

3.9.4. Lemma. Over an algebraically closed field $k$ of characteristic $p \neq l$, the object $K$ on $\mathbb{A}^{m}, m \geq 1$, given by

$$
K:=R j_{*} \overline{\mathbb{Q}}_{l}[2 m-1] \text { for } j: \mathbb{A}^{m}-\{0\} \rightarrow \mathbb{A}^{m} \text { the inclusion, }
$$

is semiperverse. 
Proof. It is trivial that $K$ is semiperverse on $\mathbb{A}^{m}-\{0\}$. We must show that the stalks $\left(\mathscr{H}^{i} K\right)_{0}$ vanish for $i>0$. Denoting by $i:\{0\} \rightarrow \mathbb{A}^{m}$ the inclusion, we have a triangle

$$
0 \rightarrow j ! \overline{\mathbb{Q}}_{l}[2 m-1] \rightarrow K \rightarrow i_{*} i^{*} K \rightarrow 0 .
$$

Since $H\left(\mathbb{A}^{m}, j_{!} \overline{\mathbb{Q}}_{l}[2 m-1]\right)=0$, the long exact cohomology sequence gives

$$
H^{i}\left(\mathbb{A}^{m}, K\right) \cong\left(\mathscr{H}^{i} K\right)_{0} ;
$$

but

$$
H^{i}\left(\mathbb{A}^{m}, K\right)=H^{i}\left(\mathbb{A}^{m}, R j_{*} \overline{\mathbb{Q}}_{l}[2 m-1]\right)=H^{i+2 m-1}\left(\mathbb{A}^{m}-\{0\}, \overline{\mathbb{Q}}_{l}\right),
$$

so we must show that

$$
H^{i}\left(\mathbb{A}^{m}-\{0\}, \overline{\mathbb{Q}}_{l}\right)=0 \text { for } i \geq 2 m ;
$$

but $\mathbb{A}^{m}-\{0\}$ is a Zariski-locally-trivial $\mathbb{G}_{m}$ bundle over $\mathbb{P}^{m-1}$, so this vanishing follows from the Leray spectral sequence

$$
E_{2}^{a, b}=H^{a}\left(\mathbb{P}^{m-1}, \overline{\mathbb{Q}}_{l}\right) \otimes H^{b}\left(\mathbb{G}_{m}, \overline{\mathbb{Q}}_{l}\right) \Rightarrow H^{a+b}\left(\mathbb{A}^{m}-\{0\}, \overline{\mathbb{Q}}_{l}\right) .
$$

(In fact, this is the standard calculation which shows that $\mathbb{A}^{m}-\{0\}$ has the same $\overline{\mathbb{Q}}_{l}$-cohomology as the standard $2 m-1$ sphere). Q.E.D.

Applying Main Theorem 1.5 gives

\subsubsection{Corollary. Suppose we are given:}

$m \geq 1$, an integer;

$k$, an algebraically closed field of characteristic $p \neq l$;

$V$, a separated $k$-scheme of finite type;

$f: V \rightarrow \mathbb{A}^{m}$, an arbitrary morphism;

$\pi: V \rightarrow \mathbb{A}^{n}$ a quasifinite morphism.

Then for any perverse $L$ on $V$, the object

$$
M(L):=R\left(p r_{2}\right)_{!}\left(p r_{1}^{*} L \otimes f_{\mathrm{aff}}^{*} R j_{*} \overline{\mathbb{Q}}_{l}[2 m-1]\right)[n m]
$$

on $\operatorname{Aff} \operatorname{Maps}\left(\mathbb{A}^{n}, \mathbb{A}^{m}\right)$ is semiperverse. Its dual is the object

$$
D M(L)=R\left(p r_{2}\right)_{*}\left(p r_{1}^{*} D L \otimes f_{\text {aff }}^{*} j_{!} \overline{\mathbb{Q}}_{l}[1](m)\right)[n m](n m) .
$$

For given $L$ there exists a dense open set $\mathscr{U}$ of $\operatorname{Aff} \operatorname{Maps}\left(\mathbb{A}^{n}, \mathbb{A}^{m}\right)$ over which both $M(L)$ and its dual have lisse cohomology sheaves, whose formation commutes with arbitrary change of base on $\mathscr{U}$. By the semiperversity of $M(L)$, the cohomology sheaves $\mathscr{H}^{i} M(L) \mid \mathscr{U}$ vanish for $i>-n m-m$. Dually, the cohomology sheaves $\mathscr{H}^{i} D M(L)$ vanish for $i<-n m-m$.

Thus if $(A, b)$ lies in $\mathscr{U}$, the stalk at $(A, b)$ of

$$
D M(L)=R\left(p r_{2}\right)_{*}\left(p r_{1}^{*} D L \otimes f_{\text {aff }}^{*} j_{!} \overline{\mathbb{Q}}_{l}[1](m)\right)[n m](n m)
$$

is

$$
R \Gamma\left(V,\left(j_{A, b}\right)_{!}\left(j_{A, b}\right)^{*} D L\right)[n m+1](n m+m)
$$


and

$$
H^{i}\left(V,\left(j_{A, b}\right)_{!}\left(j_{A, b}\right)^{*} D L\right)[n m+1]=0 \text { for } i<-n m-m .
$$

In other words, for $(A, b)$ in $\mathscr{U}$, we find that

$$
H^{i}\left(V,\left(j_{A, b}\right)_{!}\left(j_{A, b}\right)^{*} D L\right)=0 \text { for } i<1-m .
$$

Exactly as above, we then deduce

3.9.6. Corollary ("Weak Lefschetz"). Suppose we are given:

$m \geq 1$, an integer;

$k$, an algebraically closed field of characteristic $p \neq l$;

$V$, a separated $k$-scheme of finite type, which is a local complete intersection, purely of dimension $d>m$;

$f: V \rightarrow \mathbb{A}^{m}$, an arbitrary morphism; and

$\pi: V \rightarrow \mathbb{A}^{n}$, a quasi-finite morphism.

Then for any lisse $\overline{\mathbb{Q}}_{l}$-sheaf $\mathscr{F}$ on $V$, there exists a dense open set $\mathscr{U}$ of $\operatorname{Aff} \operatorname{Maps}\left(\mathbb{A}^{n}, \mathbb{A}^{m}\right)$ such that, if $(A, b)$ in $\mathscr{U}$, then

(1) $H^{a}\left(V,\left(j_{A, b}\right)_{!}\left(j_{A, b}\right)^{*} \mathscr{F}\right)=0$ for $a<d+1-m$,

(2) the restriction map

$$
H^{a}(V, \mathscr{F}) \rightarrow H^{a}\left(V \cap\left(f_{A, b}=0\right),\left(i_{A, b}\right)^{*} \mathscr{F}\right)
$$

is an isomorphism for $a<d-m$ and injective for $a=d-m$.

Exactly as above, this leads immediately to

3.9.7. Corollary ("Weak Bertini"). Suppose we are given:

$k$, an algebraically closed field of characteristic $p \neq l$;

$V$, a separated $k$-scheme of finite type, which is a connected local complete intersection, purely of dimension $d>m$;

$f: V \rightarrow \mathbb{A}^{m}$, an arbitrary morphism;

$\pi: V \rightarrow \mathbb{A}^{n}$, a quasi-finite morphism.

Then for any finite étale covering $\rho: E \rightarrow V$ with $E$ connected, there exists a dense open set $\mathscr{U}$ of $\operatorname{Aff} \operatorname{Maps}\left(\mathbb{A}^{n}, \mathbb{A}^{m}\right)$ such that if $(A, b)$ in $\mathscr{U}$ then the pullback $\rho^{-1}\left(V \cap\left(f_{A, b}=0\right)\right)$ of $E$ to $V \cap\left(f_{A, b}=0\right)$

$$
\begin{array}{cc}
\rho^{-1}\left(V \cap\left(f_{A, b}=0\right)\right) & \subset E \\
\downarrow & \downarrow \\
V \cap\left(f_{A, b}=0\right) & \subset V
\end{array}
$$

is connected.

In view of Corollary 3.8.1 we find

3.9.8. Corollary ("Weak Smooth Bertini"). Suppose we are given:

$k$, and algebraically closed field of characteristic $p \neq l$;

$V$, an irreducible smooth separated $k$-scheme of finite type, of dimension $d>m$

$f: V \rightarrow \mathbb{A}^{m}$, an arbitrary morphism;

$\pi: V \rightarrow \mathbb{A}^{n}$, an unramified morphism. 
Then:

(1) There exists a dense set $\mathscr{U}$ of $\operatorname{Aff} \operatorname{Maps}\left(\mathbb{A}^{n}, \mathbb{A}^{m}\right)$ such that if $(A, b)$ in $\mathscr{U}$ then $V \cap\left(f_{A, b}=0\right)$ is smooth and irreducible.

(2) For any finite étale covering $\rho: E \rightarrow V$ with $E$ irreducible, there exist a dense open set $\mathscr{U}$ of $\operatorname{Aff} \operatorname{Maps}\left(\mathbb{A}^{n}, \mathbb{A}^{m}\right)$ such that if $(A, b)$ in $\mathscr{U}$ then the pullback $\rho^{-1}\left(V \cap\left(f_{A, b}=0\right)\right)$ of $E$ to $V \cap\left(f_{A, b}=0\right)$

$$
\begin{array}{cc}
\rho^{-1}\left(V \cap\left(f_{A, b}=0\right)\right) & \subset E \\
\downarrow & \downarrow \\
V \cap\left(f_{A, b}=0\right) & \subset V
\end{array}
$$

is smooth and irreducible.

3.10. Applications to complete intersections in $\mathbb{A}^{n}$. In the particular case when $V$ is $\mathbb{A}^{n}$ itself, we can exploit the fact that $H^{a}\left(\mathbb{A}^{n}, \overline{\mathbb{Q}}_{l}\right)=0$ for $a \neq 0$.

3.10.1. Theorem (complete intersections in $\mathbb{A}^{n}$ ). Suppose we are given:

$n>m \geq 1$, integers;

$k$, an algebraically closed field of characteristic $p \neq l$;

$m$ polynomials $f_{1}, \ldots, f_{m}$ in $k\left[x_{1}, \ldots, x_{n}\right]$, viewed as a morphism $f=\left(f_{1}, \ldots, f_{m}\right): \mathbb{A}^{n} \rightarrow \mathbb{A}^{m}$.

Then there exists a dense open set $\mathscr{U}$ of $\operatorname{Aff} \operatorname{Maps}\left(\mathbb{A}^{n}, \mathbb{A}^{m}\right)$ such that if $\left(A=\left(a_{i, j}\right), b=\left(b_{i}\right)\right)$ in $\mathscr{U}$, then the subscheme $f_{A, b}=0$ of $\mathbb{A}^{n}$ defined by the $m$ equations

$$
f_{i}\left(x_{1}, \ldots, x_{n}\right)+\sum_{j} a_{i, j} x_{j}+b_{i}=0, \quad i=1, \ldots, m,
$$

satisfies

(1) $f_{A, b}=0$ is smooth and irreducible of dimension $n-m$;

(2) its cohomology groups $H^{a}\left(\left(f_{A, b}=0\right), \overline{\mathbb{Q}}_{l}\right)$ vanish for $a \neq 0, n-m$, and

$$
H^{0}\left(\left(f_{A, b}=0\right), \overline{\mathbb{Q}}_{l}\right)=\overline{\mathbb{Q}}
$$

(3) its compact cohomology groups $H_{c}^{a}\left(\left(f_{A, b}=0\right), \overline{\mathbb{Q}}_{l}\right)$ vanish for $a \neq n-m, 2(n-m)$, and

$$
H_{c}^{2(n-m)}\left(\left(f_{A, b}=0\right), \overline{\mathbb{Q}}_{l}\right)=\overline{\mathbb{Q}}_{l}(m-n) .
$$

Proof. Statement (1) is Weak Smooth Bertini 3.9.8, applied with $V=\mathbb{A}^{n}, \pi$ the identity. Once we know (1), then by the cohomological dimension of affines, we see that $H^{a}\left(\left(f_{A, b}=0\right), \overline{\mathbb{Q}}_{l}\right)$ vanishes for $a>n-m$. Then statement (2) is Weak Lefschetz 3.9.6, applied with $V=\mathbb{A}^{n}, \pi$ the identity, and $\mathscr{F}=\overline{\mathbb{Q}}_{l}$. Statement (3) is the Poincaré dual of (2). Q.E.D.

3.10.2. Corollary (numbers of points on general complete intersections). Hypotheses and notation are as in Theorem 3.10.1 above. Let $k_{0}$ be a finite subfield of $k$, and assume that polynomials $f_{1}, \ldots, f_{m}$ in $k\left[x_{1}, \ldots, x_{n}\right]$ lie in 
$k_{0}\left[x_{1}, \ldots, x_{n}\right]$. Then there exists a constant $C$ and a dense open set $\mathscr{U}_{0}$, defined over $k_{0}$, of $\mathrm{Aff} \operatorname{Maps}\left(\mathbb{A}^{n}, \mathbb{A}^{m}\right)$ such that, if $E$ is a finite extension of $k_{0}$ inside $k$, with $\operatorname{Card}(E):=q_{E}$, and if $\left(A=\left(a_{i, j}\right), b=\left(b_{i}\right)\right)$ is an E-valued point of $\mathscr{U}_{0}$, then the subscheme $f_{A, b}=0$ of $\mathbb{A}_{E}^{n}$ defined by the $m$ equations

$$
f_{i}\left(x_{1}, \ldots, x_{n}\right)+\sum_{j} a_{i, j} x_{j}+b_{i}=0, \quad i=1, \ldots, m,
$$

is smooth and irreducible of dimension $n-m$, and its number of $E$-valued points satisfies the estimate

$$
\left|\operatorname{Card}\left(\left(f_{A, b}=0\right)(E)\right)-\left(q_{E}\right)^{n-m}\right| \leq C\left(q_{E}\right)^{(n-m) / 2} .
$$

Proof. With no loss of generality, we may suppose that $k$ is an algebraic closure of the finite field $k_{0}$. Consider the dense open set $\mathscr{U}$ of the theorem. Since $\mathscr{U}$ is defined over $k$, it descends to a dense open set $\mathscr{U}_{1}$ defined over some finite extension $k_{1}$ of $k_{0}$. We take for $\mathscr{U}_{0}$ the intersection of the finitely many $\operatorname{Gal}\left(k_{1} / k_{0}\right)$-conjugates of $\mathscr{U}_{1}$. Then $\mathscr{U}_{0}$ is a dense open set such hat $\mathscr{U}_{0} \otimes k$ lies in the open set $\mathscr{U}$ of the theorem.

At the expense of shrinking $\mathscr{U}_{0}$, we may further assume that the groups $H_{c}^{n-m}\left(\left(f_{A, b}=0\right), \overline{\mathbb{Q}}_{l}\right)$ are the stalks of a lisse sheaf on $\mathscr{U}_{0}$, and so their dimensions are independent of the particular point $(A, b)$ in $\mathscr{U}_{0}$. We claim this common dimension "works" as $C$. This results from the Lefschetz Trace Formula and the fact [De, 3.3.4] that $H_{c}^{n-m}\left(\left(f_{A, b}=0\right), \overline{\mathbb{Q}}_{l}\right)$ is mixed of weight $\leq n-m$. Q.E.D.

3.10.3. Remark. One can rechoose the constant $C$ above to depend only on the three integers

$$
n, m, d:=\max \left(1, \max _{i}\left(\operatorname{degree}\left(f_{i}\right)\right)\right) .
$$

Indeed, consider the universal situation of $m$ polynomials $f_{i, \text { univ }}$ in $n$ variables, each of degree at most $d$, with indeterminate coefficients. Denote by $R$ the polynomial ring over $\mathbb{Z}$ on these indeterminate coefficients, and by

$$
f_{\text {univ }}: \mathbb{A}_{R}^{n} \rightarrow \mathbb{A}_{R}^{m}
$$

the morphism defined by the $f_{i \text {, univ }}$. For each prime $l$, the sheaves $R^{j}\left(f_{\text {univ }}\right)_{!} \overline{\mathbb{Q}}_{l}$ on $\mathbb{A}_{R[1 / l]}^{m}$ are constructibe and vanish for $j>2 n$ or $j<0$. Let $C_{j, l}$ be the largest dimension of a stalk of $R^{j}\left(f_{\text {univ }}\right) \overline{\mathbb{Q}}_{l}$ at any geometric point of $\mathbb{A}_{R[1 / l]}^{m}$. Denote by $C_{l}$ the sum $\sum_{j} C_{j, l}$. Then $C_{l}$ certainly "works" as a $C$ in the above corollary as long as we are not in characteristic $l$, since it bounds the sum of all the $l$-adic Betti numbers of any of the varieties $f_{A, b}=0$. So we may take for $C$ the $\max \left(C_{l_{1}, l_{2}}\right)$ for any two distinct primes $l_{1}$ and $l_{2}$, e.g., 2 and 3. (It is not known that $C_{l}$ itself is independent of $l$.)

3.11. Application of Bertini to $l$-adic sheaves. We can also give a version of Bertini adapted to $l$-adic sheaves. We first make a definition. Suppose we are given a connected scheme $X$, a connected scheme $Y$, and a morphism 
$\varphi: Y \rightarrow X$. Let $l$ be a prime, $E_{\lambda}$ a finite extension of $\mathbb{Q}_{l}, \mathscr{O}_{\lambda}$ the ring of integers in $E_{\lambda}$, and $A$ one of the rings $E_{\lambda}, \mathscr{O}_{\lambda}$, or $\mathscr{O}_{\lambda} / \lambda^{\nu} \mathscr{O}_{\lambda}$ for some integer $\nu \geq 1$. Let $\mathscr{F}$ be a lisse $A$-sheaf on $X$, corresponding, for each choice of geometric point $x$ in $X$, to a continuous homomorphism

$$
\rho_{\mathscr{F}}: \pi_{1}(X, x) \rightarrow \operatorname{Aut}_{A}\left(\mathscr{F}_{X}\right) .
$$

We say that $\mathscr{F}$ on $X$ and $\varphi^{-1}(\mathscr{F})$ on $Y$ have the same monodromy if for some (or equivalently for every) geometric point $y$ of $Y$, the composite homomorphism $\varphi_{*} \circ \rho_{\mathscr{F}}$

$$
\pi_{1}(Y, y) \rightarrow \pi_{1}(X, \varphi(y)) \rightarrow \operatorname{Aut}_{A}\left(\mathscr{F}_{\varphi(y)}\right),
$$

which "is" $\varphi^{-1}(\mathscr{F})$, has the same image in $\operatorname{Aut}_{A}\left(\mathscr{F}_{\varphi(y)}\right)$ as $\rho_{\mathscr{F}}$.

3.11.1. Theorem ("l-adic Bertini"). Suppose we are given:

$k$, an algebraically closed field of characteristic $p \neq 0$;

$V$, a separated $k$-scheme of finite type, which is a connected local com-

plete intersection, purely of dimension $d>m$;

$f: V \rightarrow \mathbb{A}^{m}$, an arbitrary morphism;

$\pi: V \rightarrow \mathbb{A}^{n}$, a quasi-finite morphism.

Fix a prime number $l$ and a finite extension $E_{\lambda}$ of $\mathbb{Q}_{l}$, and let $R$ be one of the rings $E_{\lambda}, \mathscr{O}_{\lambda}$, or $\mathscr{O}_{\lambda} / \lambda^{\nu} \mathscr{O}_{\lambda}$ for some integer $\nu \geq 1$. Let $\mathscr{F}$ be a lisse $R$-sheaf on $X$. Then there exists a dense open set $\mathscr{U}$ of $\operatorname{Aff} \operatorname{Maps}\left(\mathbb{A}^{n}, \mathbb{A}^{m}\right)$ such that if $(A, b)$ in $\mathscr{U}$ then the pullback $\mathscr{F} \mid V \cap\left(f_{A, b}=0\right)$ has the same monodromy as $\mathscr{F}$.

Proof. Suppose first that $R$ is $\mathscr{O}_{\lambda} / \lambda^{\nu} \mathscr{O}_{\lambda}$ for some integer $\nu \geq 1$. Then $\mathscr{F}$ has

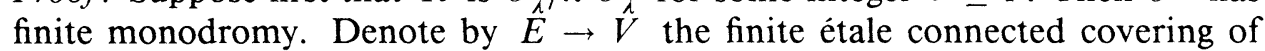
$V$, which trivializes $\mathscr{F}$. Then the assertion is precisely that the pullback of $E$ to $V \cap\left(f_{A, b}=0\right)$ remains connected, for $(A, b)$ in a dense open set $\mathscr{U}$ of $\operatorname{Aff} \operatorname{Maps}\left(\mathbb{A}^{n}, \mathbb{A}^{m}\right)$, and this is precisely Weak Bertini.

The case when $R$ is $E_{\lambda}$ results from the case when $A$ is $\mathscr{O}_{\lambda}$, since any lisse $E_{\lambda}$-sheaf has an $\mathscr{O}_{\lambda}$-form. When $R$ is $\mathscr{O}_{\lambda}$, we apply Pink's Lemma [Ka1, 8.18.3] to the image of $\rho_{\mathscr{F}}$, to reduce to the case when $R$ is an $\mathscr{O}_{\lambda} / l^{\nu} \mathscr{O}_{\lambda}$. Q.E.D.

\section{Diophantine applications}

4.0. Diophantine applications to individual exponential sums. In this section, we combine our semiperversity results with Deligne's Weil II estimate [De, 3.3.1] to give good estimates for the archimedean absolute values of sufficiently general exponential sums in the families we consider and to give moderately good estimates for the average archimedean absolute values of all the sums in these families. In the following sections, we will give sharper estimates for average absolute values, at the expense of imposing hypotheses that are not so easy to verify in practice.

We begin by stating explicitly the diophantine corollary of Main Theorem 1.5 . 
4.0.1. Diophantine version of the main theorem. In the notation of Main Theorem 1.5, suppose we are given:

$k$, a finite field of characteristic $p \neq l$;

$V$, a separated $k$-scheme of finite type;

$f: V \rightarrow \mathbb{A}^{m}$, an arbitrary morphism;

$\pi: V \rightarrow \mathbb{A}^{n}$, a quasi-finite morphism;

$K$, semiperverse on $\mathbb{A}^{m}$ and mixed of weight $\leq \alpha$;

$L$, semiperverse on $V$ and mixed of weight $\leq \beta$.

Suppose further that either $H_{c}\left(\mathbb{A}^{m} \otimes \bar{k}, K\right)=0$ or $H_{c}(V \otimes \bar{k}, L)=0$. Then the object $M:=R\left(p r_{2}\right)_{!}\left(p r_{1}^{*} L \otimes f^{*}{ }_{\text {aff }} K\right)[n m]$ on $\operatorname{Aff} \operatorname{Maps}\left(\mathbb{A}^{n}, \mathbb{A}^{m}\right)$ is semiperverse, mixed of weight $\leq n m+\alpha+\beta$, and

$$
H_{c}\left(\operatorname{Aff} \operatorname{Maps}\left(\mathbb{A}^{n}, \mathbb{A}^{m}\right) \otimes \bar{k}, M\right)=0 \text {. }
$$

Proof. The only new statement concerns weights. The object

$$
\left(p r_{1}^{*} L \otimes f_{\text {aff }}^{*} K\right)[n m]
$$

is mixed of weight $\leq n m+\alpha+\beta$, and by [De, 3.3.1] this property is preserved by the operator $R\left(p r_{2}\right)_{\text {! }}$. Q.E.D.

4.1. In order to make explicit the application to sums, we introduce the following notation. Given a separated $k$-schemes of finite type $X$, an object $K$ of $D_{c}^{b}\left(X, \overline{\mathbb{Q}}_{l}\right)$, a finite extension field $E$ of $k$, and an $E$-valued point $x$ of $X$, we denote by $K(x, E)$ in $\overline{\mathbb{Q}}_{l}$ the number

$$
K(x, E):=\sum_{i}(-1)^{i} \operatorname{Trace}\left(F_{x, E} \mid \mathscr{H}^{i}(K)_{\bar{x}}\right) .
$$

For each fixed $E$, we call the $\overline{\mathbb{Q}}_{1}$-valued function on $X(E)$ given by $x \mapsto$ $K(x, E)$ the "trace function of $K$ ". The interplay between semiperversity and mixedness is given by

4.1.1. Proposition. Let $k$ be a finite field of characteristic $\neq l, X$ a separated $k$-scheme of finite type and dimension $\leq d$, and $K$ an object in $D_{c}^{b}\left(X, \overline{\mathbb{Q}}_{l}\right)$, which is semiperverse and mixed weight $\leq w$. Then:

(1) There exists constants $C_{1}$ and $C_{2}$ such that for all finite extensions $E$ of $k$, we have the $L^{2}$ estimate

$$
\sum_{x \text { in } X(E)}|K(x, E)|^{2} \leq C_{2}\left(q_{E}\right)^{w}
$$

and the $L^{1}$ estimate

$$
\sum_{x \text { in } X(E)}|K(x, E)| \leq\left(C_{1} C_{2}\right)^{1 / 2}\left(q_{E}\right)^{(w+d) / 2} .
$$

(2) If $X$ is equidimensional of dimension $d$, there exists a dense open set $U$ in $X$ and a constant $C$, such that for any finite extension field $E$ of $k$, of cardinality $q_{E}$, and any $E$-valued point $u$ of $U$, we have the estimate

$$
|K(u, E)| \leq C\left(q_{E}\right)^{(w-\operatorname{dim}(X)) / 2} .
$$


Proof. (1) Let $X^{\text {red }}=\amalg Z_{\gamma}$ be a finite partition of $X^{\text {red }}$ into a disjoint union of smooth connected subschemes $Z_{\gamma}, \operatorname{dim}\left(Z_{\gamma}\right):=d_{\gamma}$, over each of which all the cohomology sheaves $\mathscr{H}^{a}(K)$ are lisse. Because $K$ is semiperverse.

$$
\mathscr{H}^{a}(K) \mid Z_{\gamma} \neq 0 \Rightarrow d_{\gamma} \leq-a \text {, i.e., } \mathscr{H}^{a}(K) \mid Z_{\gamma} \neq 0 \Rightarrow a \leq-d_{\gamma} .
$$

Since $K$ is mixed of weight $\leq w$,

$$
\mathscr{H}^{a}(K)\left|Z_{\gamma} \neq 0 \Rightarrow \mathscr{H}^{a}(M)\right| Z_{\gamma} \text { mixed of weight } \leq w-d_{\gamma} .
$$

For each $Z_{\gamma}$, denote by $C_{\gamma}$ the sum (over $a$ ) of the ranks of the lisse sheaves $\mathscr{H}^{a}(K) \mid Z_{\gamma}$. Then for any finite extension $E$ of $k$ and any $E$-valued point $z$ of $Z_{\gamma}$, we have the obvious estimate

$$
|K(z, E)| \leq C_{\gamma}\left(q_{E}\right)^{\left(w-d_{\gamma}\right) / 2} \text { if } z \text { in } Z_{\gamma}(E) .
$$

Squaring, we get

$$
|K(z, E)|^{2} \leq C_{\gamma}^{2}\left(q_{E}\right)^{\left(w-d_{\gamma}\right)} \quad \text { if } z \text { in } Z_{\gamma}(E) .
$$

Taking the sum over all the $E$-valued points of $X$, we find

$$
\sum_{x \text { in } X(E)}|K(x, E)|^{2} \leq \sum_{\gamma} \operatorname{Card}\left(Z_{\gamma}(E)\right) C_{\gamma}^{2}\left(q_{E}\right)^{\left(w-d_{\gamma}\right)} .
$$

Since $Z_{\gamma}$ has dimension $d_{\gamma}$, there exists a constant $D_{\gamma}$ such that for any finite extension $E$ of $k, \operatorname{Card}\left(Z_{\gamma}(E)\right) \leq D_{\gamma}\left(q_{E}\right)^{d_{\gamma}}$. So the above estimate becomes

$$
\sum_{x \text { in } X(E)}|K(x, E)|^{2} \leq \sum_{\gamma} D_{\gamma} C_{\gamma}^{2}\left(q_{E}\right)^{w} .
$$

We take $C_{2}:=\sum_{\gamma} D_{\gamma} C_{\gamma}^{2}$, which gives the $L^{2}$ estimate. The $L^{1}$ estimate follows from this by Cauchy Schwartz, once we pick a constant $C_{1}$ such that for any finite extension $E$ of $k$, we have

$$
\operatorname{Card}(X(E)) \leq C_{1}\left(q_{E}\right)^{d} .
$$

(2) Let $U$ be a dense open set of $X$ over which all the cohomology sheaves $\mathscr{H}^{i}(K) \mid U$ are lisse and denote by $C$ the sum (over $i$ ) of the ranks of all the lisse sheaves $\mathscr{H}^{i}(K) \mid U$. Since $K$ is semiperverse and $U$ is equidimensional of dimension $d$, we see

$$
\mathscr{H}^{i}(K) \mid U=0 \text { if } i>-d .
$$

Since $K$ is mixed of weight $\leq w$, each sheaf $\mathscr{H}^{i}(K)$ is mixed of weight $\leq$ $i+w$. So on $U$, the only possibly nonvanishing cohomology sheaves $\mathscr{H}^{i}(K) \mid U$ are mixed of weight $\leq w-d$, and the sum of their ranks is $C$. So for the trace function of $K$ we have the estimate

$$
|K(u, E)| \leq C\left(q_{E}\right)^{(w-d) / 2} \text { if } u \text { in } U(E) \text {. Q.E.D. }
$$

We now return to the situation of Theorem 4.0.1. 
4.1.2. Corollary. Hypotheses and notations are as in Theorem 4.0.1. We have:

(1) There exists a dense open set $\mathscr{U}$ of $\operatorname{Aff} \operatorname{Maps}\left(\mathbb{A}^{n}, \mathbb{A}^{m}\right)$ and a constant $C$, such that for any finite extension field $E$ of $k$, of cardinality $q_{E}$, and any $E$-valued point $(A, b)$ of $\mathscr{U}$, the sum

$$
S((A, b), E):=\sum_{v \text { in } V(E)} L(v, E) \times K(f(v)+A \pi(v)+b, E)
$$

satisfies the estimate

$$
|S((A, b), E)| \leq C\left(q_{E}\right)^{(\alpha+\beta-m) / 2} .
$$

(2) There exists a constant $C_{2}$ such that for any finite extension field $E$ of $k$, of cardinality $q_{E}$, the absolute values $|S((A . b), E)|$ at the E-valued points $(A, b)$ of $\operatorname{Aff} \operatorname{Maps}\left(\mathbb{A}^{n}, \mathbb{A}^{m}\right)$ satisfy the $L^{2}$ estimate

$$
\left(1 /\left(q_{E}\right)^{n m+m}\right) \sum_{\text {all } E \text {-valued }(A, b)}|S((A . b), E)|^{2} \leq C_{2}\left(q_{E}\right)^{\alpha+\beta-m},
$$

and the $L^{1}$ estimate

$$
\left(1 /\left(q_{E}\right)^{n m+m}\right) \sum_{\text {all } E \text {-valued }(A, b)}|S((A . b), E)| \leq C_{2}^{1 / 2}\left(q_{E}\right)^{(\alpha+\beta-m) / 2} .
$$

Proof. This is just the previous proposition, applied to the object $M:=$ $R\left(p r_{2}\right)_{!}\left(p r_{1}^{*} L \otimes f_{\text {aff }}^{*} K\right)[n m]$ on Aff Maps $\left(\mathbb{A}^{n}, \mathbb{A}^{m}\right)$, which is semiperverse and mixed of weight $\leq w:=n m+\alpha+\beta$. By the Lefschetz Trace Formula, we have

$$
M((A, b), E)=(-1)^{n m} S((A . b), E) .
$$

(2) Again the previous proposition, together with the observation that for the affine space $X:=\operatorname{Aff} \operatorname{Maps}\left(\mathbb{A}^{n}, \mathbb{A}^{m}\right)$, the constant $C_{1}$ of that proposition may be taken $=1$. Q.E.D.

Let us make this very concrete.

4.1.3. Corollary. In the notation of Main Theorem 1.5, suppose we are given:

$k$, a finite field of characteristic $p \neq l$;

$V$, a separated $k$-scheme of finite type;

$f: V \rightarrow \mathbb{A}^{m}$, an arbitrary morphism;

$\pi: V \rightarrow \mathbb{A}^{n}$, a quasi-finite morphism;

$\mathscr{F}$, a $\overline{\mathbb{Q}}_{1}$-sheaf on $\mathbb{A}^{m}$, mixed of weight $\leq a$;

$\mathscr{G}, a \overline{\mathbb{Q}}_{1}$-sheaf on $V$, mixed of weight $\leq b$.

Suppose further that either $H_{c}\left(\mathbb{A}^{m} \otimes \bar{k}, \mathscr{F}\right)=0$ or $H_{c}(V \otimes \bar{k}, \mathscr{G})=0$. We have:

(1) There exists a dense open set $\mathscr{U}$ of $\operatorname{Aff} \operatorname{Maps}\left(\mathbb{A}^{n}, \mathbb{A}^{m}\right)$ and a constant $C$, such that for any finite extension field $E$ of $k$, of cardinality $q_{E}$, and any E-valued point $(A, b)$ of $\mathscr{U}$, the sum

$$
S((A . b), E):=\sum_{v \text { in } V(E)} \mathscr{G}(v, E) \times \mathscr{F}(f(v)+A \pi(v)+b, E)
$$


satisfies the estimate

$$
|S((A . b), E)| \leq C\left(q_{E}\right)^{(a+b+\operatorname{dim}(V)) / 2} .
$$

(2) There exists a constant $C_{2}$ such that for any finite extension field $E$ of $k$, of cardinality $q_{E}$, the absolute values $|S((A . b), E)|$ at the E-valued points $(A, b)$ of $\operatorname{Aff} \operatorname{Maps}\left(\mathbb{A}^{n}, \mathbb{A}^{m}\right)$ satisfy the $L^{2}$ estimate

$$
\left(1 /\left(q_{E}\right)^{n m+m}\right) \sum_{\text {all } E \text {-valued }(A, b)}|S((A . b), E)|^{2} \leq C_{2}\left(q_{E}\right)^{\alpha+\beta+\operatorname{dim}(V)}
$$

and the $L^{1}$-estimate

$$
\left(1 /\left(q_{E}\right)^{n m+m}\right) \sum_{\text {all } E \text {-valued }(A, b)}|S((A . b), E)| \leq C_{2}^{1 / 2}\left(q_{E}\right)^{(\alpha+\beta+\operatorname{dim}(V)) / 2} .
$$

Proof. Simply apply the previous result to $K:=\mathscr{F}[m]$, which is semiperverse and mixed of weight $\leq a+m$, and to $L:=\mathscr{G}[\operatorname{dim}(V)]$, which is semiperverse and mixed of weight $\leq b+\operatorname{dim}(V)$. Q.E.D.

4.4. Examples of sheaves on $\mathbb{A}^{1}$ with $H_{c}=0$. Let us consider in greater detail the special case $m=1$ of the above estimates. It is sometimes convenient to fix a single sheaf $\mathscr{F}$ on $\mathbb{A}^{1}$, which satisfies $H_{c}\left(\mathbb{A}^{1} \otimes \bar{k}, \mathscr{F}\right)=0$ and is mixed of weight $\leq w$, and to regard all the rest of the data $(V, v, \pi, \mathscr{G})$ as variable. The following is a short partial list of such sheaves $\mathscr{F}$ 's on $\mathbb{A}^{1}$.

Example 1. $\mathscr{F}=\mathscr{L}_{\psi}$, for $\psi$ any nontrivial additive character $\psi$. Here $w=$ 0 .

Example 2. $\mathscr{F}=j_{1} \mathscr{L}_{\chi}$, for $\chi$ a nontrivial multiplicative character, and $j: \mathbb{G}_{m} \rightarrow \mathbb{A}^{1}$ the inclusion. Here again, $w=0$.

Example 3. $\mathscr{F}=j_{*} \mathscr{H}_{\lambda}(!, \psi ; \chi$ 's, $\rho$ 's $)$ with $j: \mathbb{G}_{m} \rightarrow \mathbb{A}^{1}$ the inclusion and $\mathscr{H}_{\lambda}\left(!, \psi^{\prime} ; \chi\right.$ 's,$\rho$ 's $)$ an irreducible hypergeometric sheaf on $\mathbb{G}_{m}$ (cf. [Ka, 8.4]) of type $(N, M)$ with $N \geq 1$, such that at least one of the $\chi$ 's is the trivial character $\mathbb{1}$. [This last condition is equivalent to the condition that $\mathscr{F}_{0}:=\mathscr{F}^{I_{0}}$ is one-dimensional, and this is in turn equivalent, by [Ka1, 8.5.3.1] to the condition $\chi\left(\mathbb{A}^{1} \otimes \bar{k}, \mathscr{F}\right)=0$. For any irreducible hypergeometric $\mathscr{H}_{\lambda}(!, \psi ; \chi$ 's, $\rho$ 's $)$, however, we have $H_{c}^{i}\left(\mathbb{A}^{1} \otimes \bar{k}, \mathscr{F}\right)=0$ for $i \neq 1$, so the vanishing of the remaining $H_{c}^{1}\left(\mathbb{A}^{1} \otimes \bar{k}, \mathscr{F}\right)$ is equivalent to the vanishing of $\chi\left(\mathbb{A}^{1} \otimes \bar{k}, \mathscr{F}\right)$.] Here $w=N+M-1$.

Example 4. Let $r \geq 1$ be an integer and $f$ a polynomial in $r$ variables, viewed as a map $f: \mathbb{A}^{r} \rightarrow \mathbb{A}^{1}$. According to [Ka4, 8.3], for $L:=\sum a_{i} x_{i}$ a sufficiently general linear form on $\mathbb{A}^{r}$, the sheaf $\mathscr{F}:=R^{r-1} f_{!} \mathscr{L}_{\psi(L)}$ on $\mathbb{A}^{1}$ has $H_{c}\left(\mathbb{A}^{1} \otimes \bar{k}, \mathscr{F}\right)=0$; for $j: U \rightarrow \mathbb{A}^{1}$, the inclusion of any dense open set on which $\mathscr{F}$ is lisse, $j^{*} \mathscr{F}$ is pure of weight $r-1$ and $\mathscr{F} \cong j_{*} j^{*} \mathscr{F}$. So here $w=-r-1$. 
4.5. Diophantine estimates for perverse irreducibles. Given a finite field $k$ of characteristic $p \neq l$, a separated $k$-scheme $X$ of finite type, and an object $K$ in $D_{c}^{b}\left(X, \overline{\mathbb{Q}}_{l}\right)$, which is perverse, we say that $K$ is geometrically irreducible if on $X \otimes \vec{k}$ it is a simple object in the abelian category of perverse sheaves on $X \otimes \bar{k}$.

4.5.1. Proposition ( $L^{2}$ estimate). Suppose we are given a finite field $k$ of characteristic $p \neq l$, a separated $k$-scheme $X$ of finite type, and an object $K$ in $D_{c}^{b}\left(X, \overline{\mathbb{Q}}_{l}\right)$, which is perverse, geometrically irreducible, and pure of weight zero. Then there exists a constant $C$ such that for any finite extension $E$ of $k$, we have

$$
\left.\left|1-\sum_{x \text { in } X(E)}\right| K(x, E)\right|^{2} \mid \leq C\left(q_{E}\right)^{-1 / 2} .
$$

Proof. Denote by $Z \subset X$ the closure in $X$ of the support of $\bigoplus_{i} \mathscr{H}^{i}(K)$. We may replace $X$ by $Z$ in the statement and thus reduce to the case when $Z=X$. We will assume henceforth that $Z=X$. By the structure theorem [BBD, 4.3.1] for perverse irreducibles, there exists an affine open set $U$ in $X$, which is smooth over $k$ and geometrically connected of some dimension $d$, such that $K \mid U$ is of the form $\mathscr{F}[d]$ for some lisse, geometrically irreducible sheaf $\mathscr{F}$ on $U$, which is pure of weight $-d$. Moreover, denoting by $j: U \rightarrow X$ the inclusion, we have $K \cong j_{! *} j^{*} K$.

Since $U$ is affine and $Z$ is separated, the map $j$ is affine; therefore, the objects $j_{!} j^{*} K$ and $R j_{*} j^{*} K$ on $Z$ are both perverse. By definition, $j_{l_{*}} j^{*} K$ is the image, in the abelian category of perverse sheaves, of the canonical "forget supports" map $j_{!} j^{*} K \rightarrow R j_{*} j^{*} K$. In particular, the map $j_{!} j^{*} K \rightarrow j_{! *} K \cong K$ is surjective in the abelian category of perverse sheaves. So if we denote by

$$
Y:=Z-U, \quad i: Y \rightarrow Z, \text { the inclusion, }
$$

we have a short exact sequence of perverse sheaves

$$
0 \rightarrow i_{*} j^{*} j_{! *} j^{*} K[-1] \rightarrow j_{!} j^{*} K \rightarrow j_{! *} j^{*} K \rightarrow 0,
$$

i.e., a short exact sequence

$$
0 \rightarrow i_{*} i^{*} K[-1] \rightarrow j_{!} j^{*} K \rightarrow K \rightarrow 0 .
$$

Since $K$ is pure of weight $0, i^{*} K$ is mixed of weight $\leq 0$, and hence $i^{*} K[-1]$ is both perverse and mixed of weight $\leq-1$.

Since $i_{*} i^{*} K[-1]$ and $j_{!} j^{*} K$ have disjoint supports, namely, $Y$ and $U$ respectively, the sum $\sum_{x \text { in } X(E)}|K(x, E)|^{2}$ breaks up as the sum of the two sums

$$
\sum_{y \text { in } Y(E)}|K[-1](y, E)|^{2}+\sum_{u \text { in } U(E)}|K(u, E)|^{2} .
$$

Because $i^{*} K[-1]$ on $Y$ is perverse and mixed of weight $\leq-1$, the first of these sums is part of the error term, thanks to the $L^{2}$ estimate of Proposition 4.1.1. Thus we are reduced to treating the second sum, over $U$. 
On $U, K$ is $\mathscr{F}[d]$ for some lisse, geometrically irreducible sheaf $\mathscr{F}$ on $U$, which is pure of weight $-d$. Because $\mathscr{F}$ is pure of weight $-d$, its "complex conjugate" is $\mathscr{F}^{\vee}(d)$; so for $u$ in $U(E)$ we have

$$
|K(u, E)|^{2}=|\mathscr{F}(u, E)|^{2}=\operatorname{End}(\mathscr{F})(d)(u, E) .
$$

By the Lefschetz Trace Formula, we have

$$
\sum_{u \text { in } U(E)}|K(u, E)|^{2}=\sum_{d \leq i \leq 2 d}(-1)^{i} \operatorname{Trace}\left(F_{E} \mid H_{c}^{i}(U \otimes \bar{k}, \operatorname{End}(\mathscr{F})(d))\right) .
$$

Because $\mathscr{F}$ is pure of some weight, $\operatorname{End}(\mathscr{F})(d)$ is pure of weight $-2 d$, so all the cohomology in dimension $<2 d$ is mixed of weight $\leq-1$ and so contributes to the error term. It remains to examine the dominant term $H_{c}^{2 d}(U \otimes \bar{k}, \operatorname{End}(\mathscr{F})(d))$. Because $\mathscr{F}$ is geometrically irreducible and $U$ is geometrically irreducible and smooth of dimension $d$, we have

$$
\begin{aligned}
& H_{c}^{2 d}(U \otimes \bar{k}, \operatorname{End}(\mathscr{F}))(d) \\
& \quad \cong \text { coinvariants of } \pi_{1}(X \otimes \bar{k}) \text { on } \operatorname{End}(\mathscr{F}) \cong \overline{\mathbb{Q}}_{l} \text {. Q.E.D. }
\end{aligned}
$$

4.5.2. Proposition ( $L^{1}$ estimate). Suppose we are given a finite field $k$ of characteristic $p \neq l$, a geometrically irreducible separated $k$-scheme $X$ of finite type, $d:=\operatorname{dim}(X)$, and an object $K$ in $D_{c}^{b}\left(X, \overline{\mathbb{Q}}_{l}\right)$, which is perverse, geometrically irreducible, and pure of weight zero. There exists a constant $C$ such that for any finite extension $E$ of $k$, we have

$$
\left(1 / \operatorname{Card}(X(E)) \sum_{x \text { in } X(E)}|K(x, E)| \leq\left(q_{E}\right)^{-d / 2}+C\left(q_{E}\right)^{-(d+1) / 2} .\right.
$$

Proof. Since $X$ is geometrically irreducible of dimension $d$, there exists a constant $D$ such that for every finite extension $E$ of $k$, we have

$$
\left|\operatorname{Card}(X(E))-\left(q_{E}\right)^{d}\right| \leq D\left(q_{E}\right)^{d-1 / 2} .
$$

The result now follows from the $L^{2}$ estimate by Cauchy Schwartz. Q.E.D.

\subsection{The situation over $\mathbb{Z}$.}

4.6.1. Let $S$ be a normal connected scheme of finite type over $\mathbb{Z}[1 / l]$ and $X / S$ a smooth $S$-scheme of relative dimension $d$, with geometrically connected fibres. For each finite field $k$ and each $k$-valued point $s$ of $S$, we denote by $X_{s}$ the fibre of $X / S$ at $s$. Thus $X_{s}$ is a smooth, geometrically connected $k$-scheme of dimension $d$.

4.6.2. Fix a real number $w$. Let $K$ be an object of $D_{c}^{b}\left(X, \overline{\mathbb{Q}}_{l}\right)$ such that for each finite field $k$ and each $k$-valued point $s$ of $S$, the object $K_{s}:=K \mid X_{s}$ on $X_{s}$ is perverse and mixed of weight $\leq w$, and there exists a dense open set $W_{s}$ of $X_{s}$ on which $K_{s}$ is geometrically irreducible and pure of weight $w$. Then for any dense open set $U_{s}$ of $X_{s}$ on which $K_{s}$ has lisse cohomology sheaves, $\mathscr{H}^{i}\left(K_{s}\right) \mid U_{s}$ vanishes for $i \neq-d$ (because $K_{s}$ is perverse), and the lisse sheaf $\mathscr{H}^{-d}\left(K_{s}\right) \mid U_{s}$ is both geometrically irreducible (because it is geometrically irreducible on the dense open set $W_{s} \cap U_{s}$ ) and pure of weight $w-d$, (because it is lisse geometrically irreducible and mixed on $U_{s}$, hence pure on $U_{s}$ of some weight, which we can read on $W_{s} \cap U_{s}$ ). 
4.6.3. Let $U \subset X$ be a dense affine open set over which all of the cohomology sheaves $\mathscr{H}^{i}(K)$ are lisse. At the expense of replacing $S$ by a dense open set of itself, we may assume that $U / S$ is surjective. So each fibre $U_{s}$ of $U / S$ is open dense in the corresponding fibre $X_{s}$ of $X / S$. As above, $\mathscr{H}^{i}\left(K_{s}\right) \mid U_{s}$ vanishes for $i \neq-d$. Therefore $\mathscr{H}^{i}(K) \mid U$ vanishes for $i \neq-d$, and hence $K \mid U \cong \mathscr{F}[d]$ for $\mathscr{F}:=\mathscr{H}^{-d}(K) \mid U$. This lisse sheaf $\mathscr{F}$ on $U$ is punctually pure of weight $w-d$, and its restriction to each finite-field fibre $U_{s}$ of $U / S$ is geometrically irreducible. We call the rank of $\mathscr{F}$ the "generic rank of $K$ ".

4.6.4. Theorem. Let $S$ be a normal connected scheme of finite type over $\mathbb{Z}[1 / l]$, whose generic point has characteristic zero, and $X / S$ a smooth $S$-scheme of relative dimension $d$, with geometrically connected fibres. Let $K$ be an object of $D_{c}^{b}\left(X, \overline{\mathbb{Q}}_{l}\right)$ and $w$ a real number, such that for each finite field $k$ and each $k$-valued point $s$ of $S$, the object $K_{s}:=K \mid X_{s}$ on $X_{s}$ is perverse and mixed of weight $\leq w$ and there exists a dense open set $U_{s}$ of $X_{s}$ on which $K_{s}$ is geometrically irreducible and pure of weight $w$. Suppose that the generic rank $r$ of $K$ is $\geq 2$. Define a real number $\alpha(r)<1$ by

$$
\alpha(r)=1-1 / 2(1+r)^{2} \text {. }
$$

Then there exists a dense open set $V$ of $S$, a constant $C$, and a finite étale galois covering $V^{\prime} \rightarrow V$ with the following property: If $k$ is a finite field $\mathbb{F}_{q}$ and $v$ is a $k$-valued point of $V$, which splits completely in the covering $V^{\prime} \rightarrow V$, then we have the estimate

$$
\left(1 / \operatorname{Card}\left(X_{v}(k)\right) \sum_{x \text { in } X_{v}(k)}|K(x, k)| \leq \alpha(r) q^{(w-d) / 2}+C q^{(w-d-1) / 2} .\right.
$$

Proof. Let $j: U \rightarrow X$ be the inclusion of a dense affine open set over which all of the cohomology sheaves $\mathscr{H}^{i}(K)$ are lisse. Shrinking $S$, we may assume that $U / S$ is surjective. Then $K \mid U \cong \mathscr{F}[d]$, for $\mathscr{F}$ a lisse sheaf on $U$ of rank $r \geq 2$, which is punctually pure of weight $w-d$, and whose restriction to each finite-field fibre $U_{s}$ of $U / S$ is geometrically irreducible. We first reduce to the case when $X$ is $U$ and $K$ is $\mathscr{F}[d]$.

Denote by $Y:=X-U$ the closed complement and by $i: Y \rightarrow X$ the inclusion. In the tautological triangle

$$
j_{!} j^{*} K \rightarrow K \rightarrow i_{*} i^{*} K
$$

the object $i_{*} i^{*} K$ is supported in $Y$. Looking fibre by fibre, we see trivially that $i^{*} K_{s}$ is semiperverse and mixed of weight $\leq w$, on each finite-field fibre $Y_{s}$. At the expense of shrinking $S$, we can [Ka4, 1.4.4] find a stratification $Y^{\text {red }}=\coprod_{\gamma} Y_{\gamma}$ of $Y^{\text {red }}$ as a finite disjoint union of subschemes $Y_{\gamma}$, each of which is smooth and surjective over $S$ with all geometric fibres equidimensional of some common dimension $d_{\gamma}$, to which $i^{*} K$ is adapted in the sense that all its cohomology sheaves are lisse on each $Y_{\gamma}$. Applying [Ka4, 3.1], (essentially a uniform version of the $L^{1}$ estimate of Proposition 4.5.2), there exists a constant $C$ such that for any finite field $k, q:=\operatorname{Card}(k)$, and any $k$-valued point $s$ of 
$S$, we have

$$
\sum_{y \text { in } Y_{s}(k)}\left|i^{*} K(y, k)\right| \leq C q^{(w+d-1) / 2}
$$

Since $X / S$ has smooth, geometrically connected fibres of dimension $d$, and $Y / S$ has all fibres of dimension $<d$, there exists a constant $D$ such that that for any finite field $k, q:=\operatorname{Card}(k)$, and any $k$-valued point $s$ of $S$, we have

$$
\begin{aligned}
\left|\operatorname{Card} X_{s}(k)-q^{d}\right| & \leq D q^{d-1 / 2}, \\
\mid \text { Card } U_{s}(k)-q^{d} \mid & \leq D q^{d-1 / 2}, \\
\mid \text { Card } X_{s}(k)-\operatorname{Card} U_{s}(k) \mid & =\operatorname{Card}\left(Y_{s}(k)\right) \leq D q^{d-1} .
\end{aligned}
$$

Thus the entire contribution of $i_{*} i^{*} K$ is absorbed in the error term, and we are reduced to treating the case when $X$ is $U$ and $K$ is $\mathscr{F}[d]$.

Denote by $f: U \rightarrow S$ the structural morphism. Further shrinking $S$, we may assume that all the sheaves

$$
R^{i} f_{!} \operatorname{End}(\mathscr{F})(d) \quad R^{i} f_{!}\left(\operatorname{End}\left(\mathscr{F}^{\otimes 2}\right)(d) \quad \text { for } 0 \leq i \leq 2 d\right.
$$

are lisse on $S$.

Having made all of these reductions, we are reduced to showing that there exists a constant $C$ and a finite étale galois covering $S^{\prime} \rightarrow S$ with the following property: If $k$ is a finite field $\mathbb{F}_{q}$ and $s$ is a $k$-valued point of $S$, which splits completely in the covering $S^{\prime} \rightarrow S$, then we have the estimate

$$
\left(1 / \operatorname{Card}\left(X_{s}(k)\right) \sum_{x \text { in } X_{s}(k)}|\mathscr{F}(x, k)| \leq \alpha(r) q^{(w-d) / 2}+C q^{(w-d-1) / 2} .\right.
$$

It will be convenient to rewrite this estimate as

$$
\left(1 / \operatorname{Card}\left(X_{s}(k)\right) \sum_{x \text { in } X_{s}(k)} q^{(d-w) / 2}|\mathscr{F}(x, k)| \leq \alpha(r)+C q^{-1 / 2}\right.
$$

and view it as a bound for the $L^{1}$ norm of the function

$$
x \text { in } X_{s}(k) \mapsto f(x):=q^{(d-w) / 2}|\mathscr{F}(x, k)|,
$$

with $X_{s}(k)$ endowed with "counting measure," normalized to give total mass one. One basis for such estimates is the following elementary lemma, whose statement we recall:

4.6.5. Lemma [Ka4, 5.1]. Let $(X, \mu)$ be a measure space, with $\mu$ a positive measure of total mass 1 . Let $f$ be a measurable $\mathbb{C}$-valued function on $X$, $\varepsilon \geq 0, E \geq 0$, and $M \geq 0$ real numbers. Suppose that

$$
|f| \leq M, \quad \int|f|^{2} d \mu \leq 1+\varepsilon, \quad \int|f|^{4} d \mu \geq 1+E-\varepsilon .
$$

Then $\int|f| d \mu \leq 1+2 \varepsilon-E / 2(1+M)^{2}$.

We now explain how to calculate the second and fourth moments of the particular function at hand. We will show that we can take $M=r$ and that, with $\varepsilon$ of the form (an explicitable constant) $q^{-1 / 2}$, we can take $E=1$. 
Because $\mathscr{F}$ is lisse of rank $r$ and is pure of weight $w-d$, we have the trivial estimate

$$
q^{(d-w) / 2}|\mathscr{F}(x, k)| \leq r .
$$

Because $\mathscr{F}$ is pure of weight $w-d$, the "complex conjugate" of $\mathscr{F}$ is $\mathscr{F}^{\vee}(d-w)$, and so for any finite field $k, q:=\operatorname{Card}(k)$, and any $k$-valued point $x$ of $X$, we have

$$
\begin{aligned}
q^{d-w}|\mathscr{F}(x, k)|^{2} & =\operatorname{End}(\mathscr{F})(x, k), \\
q^{2 d-2 w}|\mathscr{F}(x, k)|^{4} & =\left(\operatorname{End}\left(\mathscr{F}^{\otimes 2}\right)(x, k) .\right.
\end{aligned}
$$

On the other hand, the number of points in $X_{s}(k)$ is $q^{d}$, up to an error bounded by $D q^{d-1 / 2}$. Thus the square of the $L^{2}$ norm is approximately

$$
q^{-d} \sum_{x \text { in } X_{s}(k)} q^{d-w}|\mathscr{F}(x, k)|^{2}=\sum_{x \text { in } X_{s}(k)} \operatorname{End}(\mathscr{F})(d)(x, k),
$$

and similarly the fourth power of the $L^{4}$ norm is approximately

$$
q^{-d} \sum_{x \text { in } X_{s}(k)} q^{2 d-2 w}|\mathscr{F}(x, k)|^{4}=\sum_{x \text { in } X_{s}(k)}\left(\operatorname{End}\left(\mathscr{F}^{\otimes 2}\right)(d)(x, k) .\right.
$$

Because $\mathscr{F}$ is pure of some weight, both $\operatorname{End}(\mathscr{F})$ and $\operatorname{End}\left(\mathscr{F}^{\otimes 2}\right)$ are pure of weight zero. By Weil II, the lisse sheaves $R^{i} f$ End $(\mathscr{F})(d)$ and $R^{i} f_{!}\left(\operatorname{End}\left(\mathscr{F}^{\otimes 2}\right)(d)\right.$ are mixed of weights $\leq-1$ for $i<2 d$. So up to an error which is uniformly $O\left(q^{-1 / 2}\right)$, the Lefschetz Trace Formula shows that the square of the $L^{2}$ norm and the fourth power of the $L^{4}$ norm are approximately

$$
\operatorname{Trace}\left(F_{s} \mid R^{2 d} f_{!} \operatorname{End}(\mathscr{F})(d)\right), \operatorname{Trace}\left(F_{s} \mid R^{2 d} f_{!}\left(\operatorname{End}\left(\mathscr{F}^{\otimes 2}\right)(d)\right),\right.
$$

respectively.

Because $\mathscr{F}$ is lisse and geometrically irreducible on each fibre $X_{s}$, the lisse sheaf $R^{2 d} f_{!}(\operatorname{End}(\mathscr{F})(d))$ has all its stalks canonically $\overline{\mathbb{Q}}_{l}$, so the square of the $L^{2}$ norm is always approximately $=1$.

It remains to analyse $\operatorname{Trace}\left(F_{s} \mid R^{2 d} f_{!}\left(\operatorname{End}\left(\mathscr{F}^{\otimes 2}\right)(d)\right)\right.$. Because $\mathscr{F}$ is lisse and geometrically irreducible on each fibre $X_{s}$, the argument of [Ka4, 5.8 and 5.8.1] shows that the lisse sheaf $R^{2 d} f_{!}\left(\operatorname{End}\left(\mathscr{F}^{\otimes 2}\right)(d)\right)$ when viewed as a representation of $\pi_{1}(S)$, factors through a finite quotient, i.e., becomes trivial on a finite étale galois covering $S^{\prime} \rightarrow S$. If we show that this representation has rank $\geq 2$, then for any finite field $k$ and any $k$-valued point $s$ of $S$, which splits completely in $S^{\prime} \rightarrow S$, the trace

$$
\operatorname{Trace}\left(F_{s} \mid R^{2 d} f_{!}\left(\operatorname{End}\left(\mathscr{F}^{\otimes 2}\right)(d)\right)\right)=\operatorname{rank}\left(R^{2 d} f_{!}\left(\operatorname{End}\left(\mathscr{F}^{\otimes 2}\right)(d)\right)\right) \geq 2,
$$

and as explained above, this will conclude the proof, with $E=1$ and $\varepsilon=$ (some explicitable constant) $q^{-1 / 2}$.

The stalk of $R^{2 d} f_{!}\left(\operatorname{End}\left(\mathscr{F}^{\otimes 2}\right)(d)\right)$ at a geometric point $s$ of $S$ is the coinvariants (or equivalently the invariants, since $\mathscr{F}_{s}$ is irreducible) of $\pi_{1}\left(X_{s}\right)$ in 
$\left(\operatorname{End}\left(\mathscr{F}_{s}^{\otimes 2}\right)\right)$. In other words, the stalk is the endomorphism ring of $\mathscr{F}_{s}{ }^{\otimes 2}$ as a $\pi_{1}\left(X_{s}\right)$-representation. Concretely, this means that when we write $\mathscr{F}_{s}^{\otimes 2}$ as the direct sum of distinct irreducible representations $V_{i}$ of $\pi_{1}\left(X_{s}\right)$ with multiplicities, say $\mathscr{F}_{s}^{\otimes 2} \cong \sum n_{i} V_{i}$, then the dimension of the stalk is $\sum\left(n_{i}\right)^{2}$. In particular, this dimension is $\geq 2$ so long as $\mathscr{F}_{s}^{\otimes 2}$ is not irreducible. But since $\mathscr{F}_{s}$ has rank $\geq 2$, the decomposition

$$
\mathscr{F}_{s}^{\otimes 2} \cong \operatorname{Sym}^{2}\left(\mathscr{F}_{s}\right) \oplus \Lambda^{2}\left(\mathscr{F}_{s}\right)
$$

has both summands nonzero and so shows that $\mathscr{F}_{s}^{\otimes 2}$ is reducible. Q.E.D.

4.7. Remarks on the theorem. One knows (cf. [Ka1, 8.18.2]) that after shrinking on $S$, for any geometric point $s$ in $S$, the conjugacy class in $\operatorname{GL}\left(r, \overline{\mathbb{Q}}_{l}\right)$ of the image $\Gamma(s)$ of $\pi_{1}\left(U_{s}\right.$, any base point $)$ in the monodromy representation of $\mathscr{F} \mid U_{s}$ is independent of the point $s$. We denote by $G$ the Zariski closure in $\operatorname{GL}\left(r, \overline{\mathbb{Q}}_{l}\right)$ of any of the groups $\Gamma(s)$. Thus $G$ is a semisimple (not necessarily connected) irreducible subgroup of $\mathrm{GL}\left(r, \overline{\mathbb{Q}}_{l}\right)$. A sufficient condition for the lisse sheaf $R^{2 d} f_{!}\left(\operatorname{End}\left(\mathscr{F}^{\otimes 2}\right)(d)\right.$ ) on $S$ to be trivial (as a representation of $\pi_{1}(S)$ ) is that for each finite field $k$ and each $k$-valued point $s$ of $S$, the image (by the monodromy representation of $\left.\mathscr{F}_{s}\right)$ of $\pi_{1}\left(U_{s}\right)$ in $\mathrm{GL}(r)$ lies inside the subgroup $\mathbb{G}_{m} G$ (cf. the proof of $[\mathrm{Ka} 4,5.8]$ ). Notice that in any case the image of $\pi_{1}\left(U_{s}\right)$ in $\mathrm{GL}(r)$ normalizes the group $\Gamma(s)$ and so normalizes its Zariski closure $G$. Thus if the quotient group

\section{(Normalizer of $G$ in $\mathrm{GL}(r)) / \mathbb{G}_{m} G$}

vanishes, then the lisse sheaf $R^{2 d} f_{!}\left(\operatorname{End}\left(\mathscr{F}^{\otimes 2}\right)(d)\right)$ on $S$ is trivial, and so in the theorem the covering $V^{\prime}$ to $V$ is completely decomposed. In this case, one gets the estimate

$$
\left(1 / \operatorname{Card}\left(X_{v}(k)\right) \sum_{x \text { in } X_{v}(k)}|K(x, k)| \leq \alpha(r) q^{(w-d) / 2}+C q^{(w-d-1) / 2}\right.
$$

for all finite fields $k$ and all $k$-valued points $v$ of $V$.

For any irreducible semisimple subgroup $G$ of $\mathrm{GL}(r)$, the quotient group (Normalizer of $G$ in $\mathrm{GL}(r)) / \mathbb{G}_{m} G$ is finite. [For it is an algebraic group in which, by [De, 1.3.11], every element is of finite order.] Some examples of irreducible semisimple $G$ inside $\mathrm{GL}(r)$ for which this quotient is trivial are those for which $G^{0}$ is $\mathrm{SL}(r)$, or if $r$ is even, $\operatorname{Sp}(r)$, or if $r$ is odd, $\mathrm{SO}(r)$.

\section{CAlCUlation OF SOME MONODROMY GROUPS}

5.0. In this section, we will calculate the geometric monodromy groups for the one-variable examples discussed in 2.2. Thus we work over a finite field $k$, we take $n=m=1, V=\mathbb{A}^{1}, \pi$ the identity map, $f: \mathbb{A}^{1} \rightarrow \mathbb{A}^{1}$ a polynomial $f(x)$ in one variable of degree $d \geq 2, \chi$ a nontrivial character of $k^{\times}, L$ the sheaf $\overline{\mathbb{Q}}_{l}[1]$ on $V=\mathbb{A}^{1}$, and $K$ the sheaf $j_{1} \mathscr{L}_{\chi}[1]$ on $\mathbb{A}^{1}$. View $\operatorname{Aff} \operatorname{Maps}\left(\mathbb{A}^{1}, \mathbb{A}^{1}\right)$ as the $\mathbb{A}^{2}$ with coordinates $(a, b)$ corresponding to $x \mapsto a x+b$, and write 
$f_{a, b}$ for the polynomial

$$
f_{a, b}(x):=f(x)+a x+b .
$$

Then $M:=R\left(p r_{2}\right)_{!}\left(p r_{1}^{*} L \otimes f_{\text {aff }}^{*} K\right)[1]$ has

$$
\mathscr{H}^{i}(M)_{a, b}=H_{c}^{i+3}\left(\mathbb{A}^{1}\left[1 /\left(f_{a, b}\right)\right] \otimes \bar{k}, \mathscr{L}_{\chi\left(f_{a, b}\right)}\right) .
$$

Over the (nonempty by 3.5$)$ open set $U$ of $(a, b)$-space where $f_{a, b}$ has all distinct zeros, $M$ has lisse cohomology sheaves [La, 2.1.4], and

$$
\begin{aligned}
& \mathscr{H}^{i}(M) \mid U=0 \text { for } 1 \neq-2, \\
& \mathscr{H}^{-2}(M) \mid U \text { has rank } d-1 .
\end{aligned}
$$

By the Lefschetz Trace Formula, the trace function of the lisse sheaf $\mathscr{H}^{-2}(M) \mid U$ is given by the following recipe: For $E$ a finite extension of $k$, $\chi_{E}$ the character $\chi \circ \operatorname{Norm}_{E / k}$ of $E^{\times}$, and $(a, b)$ in $U(E)$, we have

$$
\operatorname{Trace}\left(F_{(a, b), E} \mid \mathscr{H}^{-2}(M)\right)=-\sum_{x \text { in } E} \chi_{E}(f(x)+a x+b) .
$$

\subsection{Determinant Lemma. Notation is as in 5.1.}

(1) If $\operatorname{char}(k) \neq 2$, denote by $\chi_{2}$ the quadratic character and by $N$ the exact order of the character $\chi \chi_{2}$. Then $\left(\operatorname{det}\left(\mathscr{H}^{-2}(M) \mid U\right)^{\otimes N}\right.$ is geometrically trivial.

(2) If $\operatorname{char}(k)=2$, denote by $N_{0}$ the (odd) order of $\chi$ and by $N:=2 N_{0}$. Then $\left(\operatorname{det}\left(\mathscr{C}^{-2}(M) \mid U\right)^{\otimes N}\right.$ is geometrically trivial.

Proof. It suffices to treat the case when $f$ is monic, for at the expense of passing to a finite extension of $k$, we may suppose that the leading coefficient of $f$ is a $d$ th power, say $\alpha^{d}$. Then pullback by the automorphism $x \mapsto x / \alpha, a \mapsto a \alpha$, $b \mapsto b$ brings us to the monic case.

It suffices now to treat the universal family $\mathscr{M}(d)$ of monic polynomials of degree $d$ with all distinct roots. Concretely, $\mathscr{M}(d)$ is the spectrum of the $k$-algebra

$$
k\left[A_{0}, A_{1}, \ldots, A_{d-1}\right][1 / \Delta]
$$

where $\Delta$ is the discriminant of the universal polynomial

$$
f_{\text {univ }}(x):=x^{d}+\sum A_{i} x^{i} .
$$

For any prime $l \neq \operatorname{char}(k)$ and $\chi$ any nontrivial $\overline{\mathbb{Q}}_{l}$-valued character of $k^{\times}$, there exists a lisse $\overline{\mathbb{Q}}_{l}$-sheaf $\mathscr{F}(d, \chi, l)$ on $\mathscr{M}(d)$ whose trace function is given by the following recipe: For $E$ a finite extension of $k, \chi_{E}$ the character $\chi \circ \operatorname{Norm}_{E / k}$ of $E^{\times}$, and $f$ in $\mathscr{M}(d)(E)$ a monic polynomial of degree $d$ over $E$ with distinct roots, we have

$$
\operatorname{Trace}\left(F_{f, E} \mid \mathscr{F}(d, \chi, l)\right)=-\sum_{x \text { in } E} \chi_{E}(f(x)) .
$$


To construct $\mathscr{F}(d, \chi, l)$, consider the sheaf $\mathscr{L}_{\chi\left(f_{\text {univ }}\right)}$ on $\left(\mathbb{A}^{1} \times \mathscr{M}(d)\right)\left[1 / f_{\text {univ }}\right]$, and the projection $p r_{2}$ onto $\mathscr{M}(d)$. The sheaves $R^{i}\left(p r_{2}\right)_{1} \mathscr{L}_{\chi\left(f_{\text {univ }}\right)}$ are all lisse [La, 2.1.4], and they vanish for $i \neq 1$, as one sees fibre by fibre. We take

$$
\mathscr{F}(d, \chi, l):=R^{1}\left(p r_{2}\right) \mathscr{L}_{\chi\left(f_{\text {univ }}\right)} \text {. }
$$

5.2bis. Determinant Lemma. (1) If $\operatorname{char}(k) \neq 2$, denote by $\chi_{2}$ the quadratic character and by $N$ the exact order of the character $\chi \chi_{2}$. Then $\left(\operatorname{det}(\mathscr{F}(d, \chi, l))^{\otimes N}\right.$ is geometrically trivial.

(2) If $\operatorname{char}(k)=2$, denote by $N_{0}$ the (odd) order of $\chi$ and by $N:=2 N_{0}$. Then $\left(\operatorname{det}(\mathscr{F}(d, \chi, l))^{\otimes N}\right.$ is geometrically trivial.

Proof. For a lisse sheaf of rank one $\mathscr{L}$ on a lisse, geometrically connected $k$ scheme $\mathscr{M}$, the short exact sequence $(\bar{\eta}$ any geometric point of $\mathscr{M})$ relating $\pi_{1}^{\text {geom }}$ and $\pi_{1}$,

$$
0 \rightarrow \pi_{1}(\mathscr{M} \otimes \bar{k}, \bar{\eta}) \rightarrow \pi_{1}(\mathscr{M}, \bar{\eta}) \rightarrow \widehat{\mathbb{Z}} \rightarrow 0,
$$

shows that $\mathscr{L}$ is geometrically constant, i.e., trivial on $\pi_{1}(\mathscr{M} \otimes \bar{k}, \bar{\eta})$, if and only if it factors through the degree map

$$
\operatorname{deg}: \pi_{1}(\mathscr{M}, \bar{\eta}) \rightarrow \widehat{\mathbb{Z}},
$$

i.e., if and only if it is of the form $\alpha^{\text {deg }}$. By Chebataroff, $\mathscr{L}$ is of the form $\alpha^{\operatorname{deg}}$ if and only if for any finite extension $E$ of $k$ and any points $x, y$ in $\mathscr{M}(E)$, we have

$$
\operatorname{Trace}\left(F_{x, E} \mid \mathscr{L}\right)=\operatorname{Trace}\left(F_{y, E} \mid \mathscr{L}\right)
$$

By [De, 1.3.4(i)] some tensor power of $\mathscr{L}$ is geometrically trivial. So for any finite extension $E$ of $k$, and any points $x, y$ in $\mathscr{M}(E)$, we have

$$
\operatorname{Trace}\left(F_{x, E} \mid \mathscr{L}\right) / \operatorname{Trace}\left(F_{y, E} \mid \mathscr{L}\right) \text { is a root of unity. }
$$

We now apply these general considerations to the situation at hand

$$
\mathscr{L}:=\operatorname{det}(\mathscr{F}(d, \chi, l)) \quad \text { on } \mathscr{M}:=\mathscr{M}(d) \text {. }
$$

The lisse sheaf $\mathscr{F}(d, \chi, l)$ has all its traces in the field $\mathbb{Q}(\chi)$. So its determinant (or indeed any sheaf obtained from $\mathscr{F}(d, \chi, l)$ by composing with a representation of $\operatorname{GL}(d-1)$ ) has all its traces in the same field $\mathbb{Q}(\chi)$. Therefore for any finite extension $E$ of $k$ and any points $x, y$ in $\mathscr{M}(d)(E)$, the ratio

$$
\operatorname{det}\left(F_{x, E} \mid \mathscr{F}(d, \chi, l)\right) / \operatorname{det}\left(F_{y, E} \mid \mathscr{F}(d, \chi, l)\right)
$$

is a root of unity, which lies in $\mathbb{Q}(\chi)$.

Let us denote by $N_{0}$ the exact order of $\chi$. Thus $\mathbb{Q}(\chi)$ is the cyclotomic field $\mathbb{Q}\left(\zeta_{N_{0}}\right)$. So the number of roots of unity in $\mathbb{Q}(\chi)$ is

$$
\operatorname{Card}\left(\mu(\mathbb{Q}(\chi))= \begin{cases}N_{0} & \text { if } N_{0} \text { is even }, \\ 2 N_{0} & \text { if } N_{0} \text { is odd }\end{cases}\right.
$$


Therefore the "mere" fact that $\mathscr{F}(d, \chi, l)$ has all its traces in the field $\mathbb{Q}(\chi)$ gives:

$$
\begin{aligned}
& \operatorname{det}(\mathscr{F}(d, \chi, l))^{\otimes N_{0}} \text { is geometrically trivial if } N_{0} \text { is even, } \\
& \operatorname{det}(\mathscr{F}(d, \chi, l))^{\otimes 2 N_{0}} \text { is geometrically trivial if } N_{0} \text { is odd. }
\end{aligned}
$$

We first treat case (2). If $\operatorname{char}(k)=2$, then $\chi$, being a character of $k^{\times}$, has odd order, and the assertion is precisely $(* *)$.

We next turn to case (1). There are two trivial cases, and one nontrivial case.

If the exact order $N_{0}$ of $\chi$ is odd, then $\chi \chi_{2}$ has exact order $2 N_{0}$, and the assertion amounts to $(* *)$. If the exact order $N_{0}$ of $\chi$ is divisible by 4 , then $\chi \chi_{2}$ has the same exact order, i.e., $N=N_{0}$ in this case, and the assertion amounts to $(*)$.

The nontrivial case is when $\chi$ is of the form $\rho \chi_{2}$, with $\rho$ a (possibly trivial) character of odd order $N_{0}$. Then $\chi \chi_{2}=\rho$ has odd order $N_{0}$. The trivial estimate $(* *)$ is that $\operatorname{det}\left(\mathscr{F}\left(d, \rho \chi_{2}, l\right)\right)^{\otimes 2 N_{0}}$ is geometrically trivial. We claim that $\operatorname{det}\left(\mathscr{F}\left(d, \rho \chi_{2}, l\right)\right)^{\otimes N_{0}}$ is geometrically trivial. In other words, we know from $(* *)$ that $\operatorname{det}\left(\mathscr{F}\left(d, \rho \chi_{2}, l\right)\right)^{\otimes N_{0}}$ is geometrically of order dividing two, and we must show that it is trivial. Concretely, this means the following: for any finite extension $E$ of $k$ and any points $f, g$ in $\mathscr{M}(d)(E)$, we know that

$$
\operatorname{det}\left(F_{f, E} \mid \mathscr{F}\left(d, \rho \chi_{2}, l\right)\right)^{N_{0}}= \pm\left(\operatorname{det}\left(F_{g, E} \mid \mathscr{F}\left(d, \rho \chi_{2}, l\right)\right)\right)^{N_{0}},
$$

and we wish to prove that

$$
\operatorname{det}\left(F_{f, E} \mid \mathscr{F}\left(d, \rho \chi_{2}, l\right)\right)^{N_{0}}=\operatorname{det}\left(F_{g, E} \mid \mathscr{F}\left(d, \rho \chi_{2}, l\right)\right)^{N_{0}} .
$$

We will prove this by a congruence argument, itself based on the possibility of varying $l$. View $\chi \quad\left(:=\rho \chi_{2}\right)$ as having values in the ring of integers $\mathscr{O}$ of the abstract cyclotomic field $\mathbb{Q}(\chi)$. For every prime $l \neq \operatorname{char}(k)$ and every $l$-adic place $\lambda$ of $\mathbb{Q}(\chi)$, we can construct a lisse $\mathscr{O}_{\lambda}$-sheaf $\mathscr{L}_{\chi\left(f_{\text {univ }}\right)}$, which yields $\mathscr{L}_{\chi\left(f_{\text {univ }}\right)}$ after extension of scalars from $\mathscr{O}_{\lambda}$ to $\overline{\mathbb{Q}}_{l}$. Denote by $\mathbb{F}_{\lambda}$ the residue field of $\mathscr{O}_{\lambda}$.

If the character $\chi$ is nontrivial $\bmod \lambda$, then the lisse [La, 2.1.4] cohomology sheaves $R^{i}\left(p r_{2}\right)_{!}\left(\mathscr{L}_{\chi\left(f_{\text {univ }}\right)} \otimes \mathbb{F}_{\lambda}\right)$ vanish for $i \neq 1$ (as one sees fibre by fibre) and $R^{1}\left(p r_{2}\right)_{!}\left(\mathscr{L}_{\chi\left(f_{\text {univ }}\right)} \otimes \mathbb{F}_{\lambda}\right)$ has rank $d-1$. A standard "universal coefficient" argument then shows that the cohomology sheaves $R^{i}\left(p r_{2}\right) ! \mathscr{L}_{\chi\left(f_{\text {univ }}\right)}$ vanish for $i \neq 1$, and the remaining

$$
\mathscr{F}(d, \chi, \lambda):=R^{1}\left(p r_{2}\right) \mathscr{L}_{\chi\left(f_{\text {univ }}\right)}
$$

is a lisse $\mathscr{O}_{\lambda}$-sheaf, $\mathscr{O}_{\lambda}$-free of rank $d-1$, which yields $\mathscr{F}(d, \chi, l)$ after extension of scalars from $\mathscr{O}_{\lambda}$ to $\overline{\mathbb{Q}}_{l}$. Moreover, we have

$$
\mathscr{F}(d, \chi, \lambda) \otimes \mathbb{F}_{\lambda} \approx R^{1}\left(p r_{2}\right)_{!}\left(\mathscr{L}_{\chi\left(f_{\text {univ }}\right)} \otimes \mathbb{F}_{\lambda}\right) \text {. }
$$

Thus once we fix a nontrivial character $\chi$ of $k^{\times}$with values in the ring of integers $\mathscr{O}$ of some cyclotomic field, for every prime number $l \neq \operatorname{char}(k)$ and 
every $l$-adic place $\lambda$ of $\mathscr{O}$ such that $\chi$ is nontrivial $\bmod \lambda$, we obtain a lisse $\mathscr{O}_{\lambda}$-sheaf $\mathscr{F}(d, \chi, \lambda)$ on $\mathscr{M}(d), \mathscr{O}_{\lambda}$-free of rank $d-1$, whose trace function is given by the usual recipe: For $E$ a finite extension of $k, \chi_{E}$ the character $\chi \circ \operatorname{Norm}_{E / k}$ of $E^{\times}$, and $f$ in $\mathscr{M}(d)(E)$ a monic polynomial of degree $d$ over $E$ with district roots, we have

$$
\operatorname{Trace}\left(F_{f, E} \mid \mathscr{F}(d, \chi, \lambda)\right)=-\sum_{x \text { in } E} \chi_{E}(f(x)),
$$

an equality in $\mathscr{O}_{i}$. The key point is that the right-hand side lies in $\mathscr{O}$, independent of $\lambda$. Therefore the determinant sheaf $\operatorname{det}(\mathscr{F}(d, \chi, \lambda))$, or indeed any sheaf deduced from $\mathscr{F}(d, \chi, \lambda)$ by a "construction of linear algebra", has all its traces in $\mathscr{O}$, independent of $\lambda$.

We now return to the problem of proving that for $\rho$ a character of odd order $N_{0}$, for any finite extension $E$ of $k$, and any points $f, g$ in $\mathscr{M}(d)(E)$, we have

$$
\operatorname{det}\left(F_{f, E} \mid \mathscr{F}\left(d, \rho \chi_{2}, l\right)\right)^{N_{0}}=\operatorname{det}\left(F_{g, E} \mid \mathscr{F}\left(d, \rho \chi_{2}, l\right)\right)^{N_{0}} .
$$

For any $l \neq \operatorname{char}(k)$ and any $l$-adic place $\lambda$ of $\mathscr{O}$ such that $\rho \chi_{2}$ is nontrivial $\bmod \lambda$ (e.g., any $l$-adic place $\lambda$ of odd residue characteristic will have $\rho \chi_{2}$ nontrivial $\bmod \lambda$, thanks to the presence of $\chi_{2}$, since already $\left(\rho \chi_{2}\right)^{N_{0}}=\chi_{2}$ is nontrivial mod such a $\lambda$ ) we may rewrite this as

$$
\operatorname{det}\left(F_{f, E} \mid \mathscr{F}\left(d, \rho \chi_{2}, \lambda\right)\right)^{N_{0}}=\operatorname{det}\left(F_{g, E} \mid \mathscr{F}\left(d, \rho \chi_{2}, \lambda\right)\right)^{N_{0}} .
$$

We proceed by induction on the number of distinct primes which divide the order $N_{0}$ of $\rho$.

To get started, we must do the crucial case when $\rho$ is trivial-we must show that $\operatorname{det}\left(\mathscr{F}\left(d, \chi_{2}, l\right)\right)$ is geometrically trivial. Over $\mathscr{M}(d)$, consider the proper smooth family of hyperelliptic curves $\varphi: \mathscr{C} \rightarrow \mathscr{M}(d)$ whose fibre over the monic polynomial $f$ of degree $d$ with all distinct roots, viewed as a point of $\mathscr{M}(d)$, is the complete nonsingular model of the affine curve $\mathscr{C}_{f}: y^{2}=f(x)$. If $d$ is odd, $\mathscr{C}$ is the disjoint union of this affine curve and of a single section “ $\infty$ ". The sheaf $\operatorname{det}\left(\mathscr{F}\left(d, \chi_{2}, l\right)\right)$ is none other than $\operatorname{det}\left(R^{1} \varphi_{!} \overline{\mathbb{Q}}_{l}\right)$. [To see this, we argue as follows. Both $\mathscr{F}\left(d, \chi_{2}, l\right)$ and $R^{1} \varphi_{!} \overline{\mathbb{Q}}_{l}$ are lisse sheaves on $\mathscr{M}(d)$ with the same trace function, namely,

$$
(f, E) \mapsto-\sum_{x} \chi_{1 / 2, E}(f(x)) .
$$

So by Chebataroff, both have isomorphic semisimplifications as lisse sheaves on $\mathscr{M}(d)$; therefore, they have isomorphic determinants.] But $R^{1} \varphi_{!} \overline{\mathbb{Q}}_{l}(1 / 2)$ carries a symplectic autoduality, the cup-product pairing on $R^{1} \varphi_{!} \overline{\mathbb{Q}}_{l}(1 / 2)$. Therefore $\operatorname{det}\left(R^{1} \varphi \overline{\mathbb{Q}}_{l}(1 / 2)\right)$ is trivial, corresponding to the fact that $\mathrm{Sp}(d-1) \subset \mathrm{Sl}(d-1)$, whence $\operatorname{det}\left(\mathscr{F}\left(d, \chi_{2}, l\right)\right) \approx \operatorname{det}\left(R^{1} \varphi \overline{\mathbb{Q}}_{l}\right)$ is geometrically trivial for $d$ odd.

If $d$ is even, we again consider the same proper smooth family of hyperelliptic curves. But this time the curve $\mathscr{C}$ is the disjoint union of the affine curve and of two disjoint sections " $\infty_{+}$" and " $\infty_{-}$", corresponding to whether the 
function $x^{d / 2} / y$ takes the value 1 or -1 . In this case, the Chebataroff argument shows that $\mathscr{F}\left(d, \chi_{2}, l\right)$ has the same semisimplification as $\overline{\mathbb{Q}}_{l} \oplus R^{1} \varphi_{!} \overline{\mathbb{Q}}_{l}$. So once again for $d$ even, $\operatorname{det}\left(\mathscr{F}\left(d, \chi_{2}, l\right)\right) \approx \operatorname{det}\left(R^{1} \varphi_{!} \overline{\mathbb{Q}}_{l}\right)$ is geometrically trivial.

We now explain how to reduce the general case $\chi=\rho \chi_{2}$ to this one. We proceed by induction on the number, say $r$, of distinct primes which divide the odd order $N_{0}$ of $\rho$. We will show that for any integer $N$ such that $\rho^{N}=\mathbb{1}$ and any $l \neq \operatorname{char}(k)$, there exists a geometrical isomorphism

$$
(* * *) \quad \operatorname{det}\left(\mathscr{F}\left(d, \rho \chi_{2}, l\right)\right)^{\otimes N} \cong \operatorname{det}\left(\mathscr{F}\left(d, \chi_{2}, l\right)\right)^{\otimes N} .
$$

Since $\operatorname{det}\left(\mathscr{F}\left(d, \chi_{2}, l\right)\right)$ is geometrically trivial, taking $N:=N_{0}$ will conclude the proof. Now $(* * *)$ holds if and only if for any finite extension $E$ of $k$ and any points $f, g$ in $\mathscr{M}(d)(E)$, we have

$$
\begin{aligned}
& \operatorname{det}\left(F_{f, E} \mid \mathscr{F}\left(d, \rho \chi_{2}, l\right)\right)^{N} / \operatorname{det}\left(F_{g, E} \mid \mathscr{F}\left(d, \rho \chi_{2}, l\right)\right)^{N} \\
& \quad=\operatorname{det}\left(F_{f, E} \mid \mathscr{F}\left(d, \chi_{2}, l\right)\right)^{N} / \operatorname{det}\left(F_{g, E} \mid \mathscr{F}\left(d, \chi_{2}, l\right)\right)^{N} .
\end{aligned}
$$

But this condition is independent of $l$, because each determinant involved lies in $\mathscr{O}$, independent of $l$. So it suffices to prove $(* * *)$ for a single choice of prime number $l \neq \operatorname{char}(k)$.

If $r \geq 1$, we can factor $\rho=\sigma \tau$ with $\sigma$ and $\tau$ characters whose odd orders are relatively prime to each other and such that $\sigma$ has odd prime power order, say $l^{e}$. Choose an $l$-adic place $\lambda$ of $\mathscr{O}$. Because $\sigma$ is trivial $\bmod \lambda$, we have an equality of $\mathbb{F}_{\lambda}$-sheaves

$$
\mathscr{F}\left(d, \sigma \tau \chi_{2}, \lambda\right) \otimes \mathbb{F}_{\lambda} \approx \mathscr{F}\left(d, \tau \chi_{2}, \lambda\right) \otimes \mathbb{F}_{\lambda} .
$$

Taking determinants, we find a congruence $\bmod \lambda$

$$
\operatorname{det}\left(\mathscr{F}\left(d, \sigma \tau \chi_{2}, \lambda\right)\right) \equiv \operatorname{det}\left(\mathscr{F}\left(d, \tau \chi_{2}, \lambda\right)\right) \bmod \lambda .
$$

Raising both sides to the $N_{0}$ th power, we conclude that

$$
\operatorname{det}\left(\mathscr{F}\left(d, \sigma \tau \chi_{2}, \lambda\right)\right)^{\otimes N_{0}} \equiv \operatorname{det}\left(\mathscr{F}\left(d, \tau \chi_{2}, \lambda\right)\right)^{\otimes N_{0}} \bmod \lambda
$$

as characters of $\pi_{1}:=\pi_{1}(\mathscr{M}(d), \bar{\eta})$. Now restrict this to $\pi_{1}^{\text {geom }}$. Since both $\operatorname{det}\left(\mathscr{F}\left(d, \sigma \tau \chi_{2}, \lambda\right)\right)^{\otimes N_{0}}$ and $\operatorname{det}\left(\mathscr{F}\left(d, \tau \chi_{2}, \lambda\right)\right)^{\otimes N_{0}}$ are \pm 1 -valued characters of $\pi_{1}^{\text {geom }}$ and $\lambda$ has odd residue characteristic, this congruence $\bmod \lambda$ implies an equality

$$
\operatorname{det}\left(\mathscr{F}\left(d, \sigma \tau \chi_{2}, \lambda\right)\right)^{\otimes N_{0}}=\operatorname{det}\left(\mathscr{F}\left(d, \tau \chi_{2}, \lambda\right)\right)^{\otimes N_{0}}
$$

as characters of $\pi_{1}^{\mathrm{geom}}$, i.e., a geometrical isomorphism

$$
\operatorname{det}\left(\mathscr{F}\left(d, \sigma \tau \chi_{2}, \lambda\right)\right)^{\otimes N_{0}} \approx \operatorname{det}\left(\mathscr{F}\left(d, \tau \chi_{2}, \lambda\right)\right)^{\otimes N_{0}} .
$$

Passing from $\mathscr{O}_{\lambda}$ to $\overline{\mathbb{Q}}_{l}$, we get a geometrical isomorphism

$$
\operatorname{det}\left(\mathscr{F}\left(d, \sigma \tau \chi_{2}, l\right)\right)^{\otimes N_{0}} \approx \operatorname{det}\left(\mathscr{F}\left(d, \tau \chi_{2}, l\right)\right)^{\otimes N_{0}} .
$$


Since $\tau^{N_{0}}=\mathbb{1}$ and the exact order of $\tau$ is divisible by fewer primes than is $N_{0}$, by induction we have a geometric isomorphism

$$
\operatorname{det}\left(\mathscr{F}\left(d, \tau \chi_{2}, \lambda\right)\right)^{\otimes N_{0}} \approx \operatorname{det}\left(\mathscr{F}\left(d, \chi_{2}, \lambda\right)\right)^{\otimes N_{0}} .
$$

Thus we have proven 5.2bis. Q.E.D.

5.3. If $\chi^{d}$ is nontrivial, then $\mathscr{H}^{-2}(M) \mid U$ is punctually pure of weight 1 . This follows from the explicit description of $\mathscr{H}^{i}(M)_{a, b}$ and [De, 3.2.3], for if we denote by

$$
j: \mathbb{A}^{1}\left[1 /\left(f_{a, b}\right)\right] \rightarrow \mathbb{P}^{1}
$$

the inclusion, then for $(a, b)$ in $U$, we have

$$
j_{!} \mathscr{L}_{\chi\left(f_{a, b}\right)}=j_{*} \mathscr{L}_{\chi\left(f_{a, b}\right)} \text { on } \mathbb{P}^{1}
$$

(This holds at all points of $\mathbb{A}^{1}$ simply in virtue of the fact that $\chi$ is nontrivial and $f_{a, b}$ has all distinct roots. That it holds at $\infty$ is equivalent to the hypothesis that $\chi^{d}$ is nontrivial.) So by the corollary, $\mathscr{H}^{-2}(M) \mid U$ as a lisse sheaf on $U$ is geometrically irreducible, provided that $\chi^{d}$ is nontrivial.

Recall (5.1) that by the Lefschetz Trace Formula, the trace function of the lisse sheaf $\mathscr{H}^{-2}(M) \mid U$ is given by the following recipe: For $E$ a finite extension of $k, \chi_{E}$ the character $\chi \circ \operatorname{Norm}_{E / K}$ of $E^{\times}$, an $(a, b)$ in $U(E)$, we have

$$
\operatorname{Trace}\left(F_{(a, b), E} \mid \mathscr{H}^{-2}(M)\right)=-\sum_{x \text { in } E} \chi_{E}(f(x)+a x+b) .
$$

Since $\mathscr{H}^{-2}(M) \mid U$ is irreducible, it is determined up to isomorphism (as lisse sheaf on $U$ ) by its trace function.

5.4. Theorem. Notation is as in 5.1. Suppose that $d$ is prime to $p$, that $f^{\prime \prime}(x)$ is not identically zero (whence $\operatorname{char}(k) \neq 2$ ), and that $\chi^{d} \neq 1$. Denote by $G_{\text {geom }}$ the geometric monodromy group of the (geometrically irreducible) lisse sheaf $\mathscr{H}^{-2}(M) \mid U$. Denote by $\chi_{2}$ the unique character of order two of $k^{\times}$, and denote by $N$ the (exact) order of the character $\chi \chi_{2}$ of $k^{\times}$. Then:

(1) If $\chi=\chi_{2}$ is the quadratic character, then $d$ is odd and $G_{\text {geom }}$ is $\operatorname{Sp}(d-1)$.

(2) If $\chi \neq \chi_{2}$, suppose that either

(a) $d>5$ or

(b) $d>3$ and $\left(\chi \chi_{2}\right)^{3} \neq 1$.

Then $\left(G_{\text {geom }}\right)^{0}=\operatorname{SL}(d-1)$ and $G_{\text {geom }}=\left\{A\right.$ in $\left.\operatorname{GL}(d-1) \mid \operatorname{det}(A)^{N}=1\right\}$.

To prove this, we need some preliminaries. 
5.5. Interlude: weakly supermorse polynomials (compare [Ka 1, 7.10.2]), convolution, and monodromy.

5.5.1. We will deduce Theorem 5.4 from a theorem about certain one-parameter families of sums. The idea is to freeze the parameter " $a$ ", consider the polynomial $f_{a}(x):=f(x)+a x$, and look at the one-parameter (namely, " $b$ ") family of sums

$$
b \mapsto S(b):=\sum_{x} \chi\left(f_{a}(x)+b\right) .
$$

It is slightly more suggestive to define

$$
g_{a}(x):=-f_{a}(x)
$$

and to write our family of sums in the form

$$
S(b)=\sum_{x} \chi\left(b-g_{a}(x)\right)=\sum_{t} \chi(b-t) \operatorname{Card}\left\{x \mid g_{a}(x)=t\right\} .
$$

Because $\sum_{t} \chi(b-t)=0, \chi$ being nontrivial, we may rewrite our family as

$$
S(b)=\sum_{t} \chi(b-t)\left[\operatorname{Card}\left\{x \mid g_{a}(x)=t\right\}-1\right] \text {. }
$$

This last expression shows that $b \mapsto S(b)$ is the additive convolution of the function $t \mapsto \chi(t)$ and the function $t \mapsto \operatorname{Card}\left\{x \mid g_{a}(x)=t\right\}-1$. The function $t \mapsto \chi(t)$ is the trace function of $j_{!} \mathscr{L}_{\chi}$ and the function

$$
t \mapsto \operatorname{Card}\left\{x \mid g_{a}(x)=t\right\}-1
$$

is the trace function of the sheaf $\mathscr{F}_{g_{a}}:=$ Kernel of Trace $g_{a}:\left(g_{a}\right)_{*} \overline{\mathbb{Q}}_{l} \rightarrow \overline{\mathbb{Q}}_{l}$. By using this convolutional point of view, we will show that already such a one-parameter family has a large $G_{\text {geom }}$ provided that the polynomial $f_{a}$, or equivalently $g_{a}$, is "weakly supermorse."

5.5.2. Given a field $k$ and a nonconstant polynomial $f(x)$ in $k[x]$ of degree $d \geq 2$, we say that $f$ is "weakly supermorse" (compare $[\mathrm{Ka} 1,7.10 .2]$ ) if the following conditions hold.

(WSM1) $d$ is invertible in $k$.

(WSM2) $f^{\prime}(x)$ has $d-1$ instinct zeros in $\bar{k}$.

(WSM3) $f$ separates the $d-1$ distinct zeros of $f^{\prime}$ in $\bar{k}$, i.e., if $f(\alpha)=f(\beta)$ and $f^{\prime}(\alpha)=f^{\prime}(\beta)=0$ then $\alpha=\beta$.

5.5.3. Suppose that $f$ is weakly supermorse, and define (compare [Ka 1, 7.10.2])

$$
\mathscr{F}:=\text { Kernel of Trace } f_{f} f_{*} \overline{\mathbb{Q}}_{l} \rightarrow \overline{\mathbb{Q}}_{l},
$$

a direct factor of $f_{*} \overline{\mathbb{Q}}_{l}$ of generic rank $d-1$. Because $f$ has degree $d$ prime to $p$ and $f$ as a map of $\mathbb{P}^{1}$ to $\mathbb{P}^{1}$ is fully ramified over $\infty, f_{*} \overline{\mathbb{Q}}_{l}$ as $I(\infty)$ representation is tame-indeed it is the direct sum

$$
f_{*} \overline{\mathbb{Q}}_{l} \text { as } I(\infty) \text {-representation } \approx \bigoplus_{\chi^{d}=\mathbb{1}} \mathscr{L}_{\chi}
$$

and hence $\mathscr{F}$ as $I(\infty)$-representation is tame, given by 


$$
\mathscr{F} \text { as } I(\infty) \text {-representation } \approx \bigoplus_{\chi^{d}=\mathbb{1}, \chi \neq \mathbb{1}} \mathscr{L}_{\chi} .
$$

Exactly as in [Ka1, 7.10.2.1 and 7.10.3], one shows that $\mathscr{F}$ is Fourier and that $\mathscr{F}$ is a geometrically irreducible tame reflection sheaf. Moreover, just as in [Kal, 7.10.4], we have

$$
\mathrm{FT}_{\psi}(\mathscr{F}[1])=\mathscr{G}[1]
$$

with $\mathscr{G}:=\operatorname{NFT}_{\psi}(\mathscr{F})$ a geometrically irreducible Fourier sheaf on $\mathbb{A}^{1}$, which is lisse on $\mathbb{G}_{m}$. Laumon's theory of the local Fourier Transform shows that

$$
\begin{aligned}
& \mathscr{G} \text { as } I(0) \text {-representation } \approx \bigoplus_{\chi^{d}=\mathbb{1}, \chi \neq \mathbb{1}} \mathscr{L}_{\chi}, \\
& \mathscr{G} \text { as } I(\infty) \text {-representation } \approx \bigoplus_{f^{\prime}(\alpha)=0} \mathscr{L}_{\chi_{2}(x)} \otimes \mathscr{L}_{\psi(f(\alpha) x)} .
\end{aligned}
$$

For any finite extension $E$ of $k$ and any $E$-valued point $t$ in $\mathbb{A}^{1}(E)=E$, the trace function of $\mathscr{F}$ at $t$ is given by

$$
\operatorname{Trace}\left(F_{t, E} \mid \mathscr{F}\right)=\operatorname{Card}\{x \text { in } E \mid f(x)=t\}-1 \text {. }
$$

So the function

$$
b \mapsto S(b):=\sum_{x} \chi(t-f(x))
$$

is the convolution of the trace functions of $\mathscr{F}$ and of $j_{1} \mathscr{L}_{\chi}$.

5.6. Lemma. If $f$ is weakly supermorse of degree $d$, and if $\chi^{d} \neq \mathbb{1}$, then $\mathscr{G} \otimes j_{1} \mathscr{L}_{\bar{\chi}}$ is a geometrically irreducible Fourier sheaf, which is tame at zero and whose $I(\infty)$-representation is given by

$$
\mathscr{G} \otimes j_{1} \mathscr{L}_{\bar{\chi}} \text { as } I(\infty) \text {-representation } \approx \bigoplus_{f^{\prime}(\alpha)=0} \mathscr{L}_{\chi_{2}(x) \tilde{\chi}(x)} \otimes \mathscr{L}_{\psi(f(\alpha) x)} .
$$

Proof. Since $\mathscr{G}$ is lisse and geometrically irreducible on $\mathbb{G}_{m}$ so is $\mathscr{G} \otimes j_{!} \mathscr{L}_{\bar{\chi}}$. Because $\chi^{d} \neq \mathbb{1}$, while the local monodromy of $\mathscr{G}$ at 0 involves only characters of order dividing $d, \mathscr{G} \otimes j_{!} \mathscr{L}_{\bar{\chi}}$ has no inertial invariants at 0 and, hence, is the direct image of its restriction to $\mathbb{G}_{m}$. Because $\mathscr{G} \otimes j_{!} \mathscr{L}_{\bar{\chi}}$ vanishes at 0 , it is not $\mathscr{L}_{\psi(\alpha x)}$ for any $\alpha$. This shows that $\mathscr{G} \otimes j_{!} \mathscr{L}_{\bar{\chi}}$ is (geometrically) irreducible Fourier. Because $\mathscr{G}$ is tame at zero so is $\mathscr{G} \otimes j_{!} \mathscr{L}_{\bar{\chi}}$. The $I(\infty)$-representation of $\mathscr{G} \otimes j_{!} \mathscr{L}_{\bar{\chi}}$ is

$$
\text { (the } I(\infty) \text {-representation of } \mathscr{G}) \otimes \mathscr{L}_{\bar{\chi}} \approx\left[\bigoplus_{f^{\prime}(\alpha)=0} \mathscr{L}_{\chi_{2}(x)} \otimes \mathscr{L}_{\psi(f(\alpha) x)}\right] \otimes \mathscr{L}_{\bar{\chi}(x)} \text {. }
$$

Q.E.D.

5.7. Key Lemma. If $f$ is weakly supermorse of degree $d$ and if $\chi^{d}=\mathbb{1}$, then:

(1) The object $\left(\mathscr{F}_{*_{!}} j_{!} \mathscr{L}_{\chi}\right)[1]$ is a geometrically irreducible Fourier sheaf, say $M$, of generic rank $d-1$.

(2) The trace function of $M$ is $t \mapsto-\sum_{x} \chi(t-f(x))$.

(3) The geometrically irreducible Fourier sheaf $\operatorname{NFT}_{\psi}(M)$ is geometrically isomorphic to $\mathscr{G} \otimes\left(j ! \mathscr{L}_{\bar{\chi}}\right)$. 
(4) $M$ is tame at $\infty$.

(5) $M$ is lisse on $\mathbb{A}^{1}$ outside the $d-1$ critical values of $f$, i.e., outside the points $f(\alpha)$ for $\alpha$ a zero of $f^{\prime}(x)$. At each critical value $f(\alpha)$, the local monodromy of $M$ is a tame pseudoreflection whose determinant, as character of $I(f(\alpha))$, is $\mathscr{L}_{\left(\chi \chi_{2}\right)(x-f(\alpha))}$.

Proof. We exploit the fact that Fourier Transform (essentially) interchanges tensor product and ! additive convolution $*_{!_{+}}:$the precise statement (cf. [ $\mathrm{Br}$, 9.6], but " $[+r g E]$ " should be " $[-r g E]$ " there) is

$$
\mathrm{FT}_{\psi}(K) \otimes \mathrm{FT}_{\psi}(L)=\mathrm{FT}_{\psi}\left(K_{* !+} L\right)[1]
$$

Taking $K$ to be $\mathscr{F}$ and $L$ to be $j_{1} \mathscr{L}_{\chi}$, and using the fact that for $\chi$ nontrivial we have

$$
\mathrm{FT}_{\psi}\left(j, \mathscr{L}_{\chi}\right)=\left(j ! \mathscr{L}_{\bar{\chi}}\right) \otimes \alpha^{\mathrm{deg}}, \quad \alpha:=-\sum_{x \text { in } k} \psi(x) \chi(x),
$$

we find

$$
\mathscr{G} \otimes\left(j ! \mathscr{L}_{\bar{\chi}}\right) \otimes \alpha^{\mathrm{deg}} \approx \mathrm{FT}_{\psi}\left(\mathscr{F}_{*_{!}} j ! \mathscr{L}_{\chi}\right)[1]
$$

Applying $\mathrm{FT}_{\bar{\psi}}(1)$ to this and using Fourier inversion, we find

$$
\mathrm{FT}_{\bar{\psi}}\left(\mathscr{G} \otimes\left(j_{!} \mathscr{L}_{\bar{\chi}}\right)(1) \otimes \alpha^{\mathrm{deg}}\right) \approx\left(\mathscr{F} *_{!+} j_{!} \mathscr{L}_{\chi}\right)([1] .
$$

Since $\mathscr{G} \otimes j ! \mathscr{L}_{\bar{\chi}}$ is geometrically irreducible Fourier, its FT is itself geometrically irreducible, i.e., $\left(\mathscr{F}_{*_{!}} j_{!} \mathscr{L}_{\chi}\right)[1]$ is geometrically irreducible Fourier. Its generic rank is the dimension, for general values of $t$, of the cohomology group on the $x$-line

$$
H_{c}^{1}\left(\mathbb{A}_{1} \otimes \bar{k}[1 /(f(x)-t)], \mathscr{L}_{\chi(t-f(x))}\right),
$$

and this group is visibly of dimension $d-1$ if $f(x)-t$ has $d$ distinct zeros. This proves (1).

Statement (2) is just the Lefschetz Trace Formula and the definition of ! additive convolution, and (3) follows from (1) by Fourier inversion.

That $M$ is tame at $\infty$ holds because $\mathscr{G} \otimes\left(j_{!} \mathscr{L}_{\bar{\chi}}\right)$ is lisse on $\mathbb{G}_{m}$, tame at 0 , and has all $\infty$-slopes $\leq 1$ (using Laumon's results, cf. [Ka1, 7.5.4]). The finite local monodromy of $M$ is as asserted by [Ka1, 7.4.6] and the previous lemma. Q.E.D.

5.8. Lemma. Over an algebraically closed field $k$, let $S$ be a finite nonempty subset of $\mathbb{A}^{1}(k), l$ a prime number invertible in $k$, and $M$ a lisse $\overline{\mathbb{Q}}_{1}$-sheaf on $\mathbb{A}^{1}-S$. Suppose that

(1) $M$ is irreducible;

(2) $M$ is tame at $\infty$;

(3) at each point $s$ in $S$, the local monodromy of $M$ is a tame pseudoreflection $\gamma_{s}$, which is not a reflection.

Then $M$ is not induced.

Proof. We argue by contradiction. Suppose that there exists a smooth connected curve $U$ over $k$, a lisse $\overline{\mathbb{Q}}_{l}$-sheaf $N$ on $X$, a finite étale map $\pi: U \rightarrow \mathbb{A}^{1}-S$ of degree $d \geq 2$, and an isomorphism $M \cong \pi_{*} N$. Denote by $X$ the complete 
nonsingular model of $U$ and by $\bar{\pi}: X \rightarrow \mathbb{P}^{1}$ the induced finite flat covering of $\mathbb{P}^{1}$. We will show that the covering $\bar{\pi}$ is tame over $\infty$ and finite étale over $\mathbb{A}^{1}$. Since $\mathbb{A}^{1}$ has no nontrivial finite étale connected coverings, which are tame at $\infty$, this gives a contradiction.

We first show that if $\pi_{*} N$ is everywhere tame, then both $N$ and $\pi$ are tame. For this, we consider the virtual sheaf of rank zero

$$
N-\operatorname{rank}(N) \overline{\mathbb{Q}}_{l} \text { on } U \text {. }
$$

The Euler-Poincaré formula on $U$ gives

$$
\chi\left(U, N-\operatorname{rank}(N) \overline{\mathbb{Q}}_{l}\right)=-\sum_{x \text { in } X-U} \operatorname{Swan}_{x}(N) .
$$

But by trivial Leray and the Euler-Poincare formula on $\mathbb{A}^{1}-S$

$$
\begin{aligned}
\chi\left(U, N-\operatorname{rank}(N) \overline{\mathbb{Q}}_{l}\right) & =\chi\left(\mathbb{A}^{1}-S, \pi_{*} N-\operatorname{rank}(N) \pi_{*} \overline{\mathbb{Q}}_{l}\right) \\
& =-\sum_{t \text { in }\{\infty, S\}} \operatorname{Swan}_{t}\left(\pi_{*} N-\operatorname{rank}(N) \pi_{*} \overline{\mathbb{Q}}_{l}\right) \\
\left(\text { since } \pi_{*} N \text { is tame }\right) & =\operatorname{rank}(N) \sum_{t \text { in }\{\infty, S\}} \operatorname{Swan}_{t}\left(\pi_{*} \overline{\mathbb{Q}}_{l}\right) .
\end{aligned}
$$

But Swan conductors are nonnegative, so the equality

$$
-\sum_{x \text { in } X-U} \operatorname{Swan}_{x}(N)=\operatorname{rank}(N) \sum_{t \text { in }\{\infty, S\}} \operatorname{Swan}_{t}\left(\pi_{*} \overline{\mathbb{Q}}_{l}\right)
$$

implies that all the terms $\operatorname{Swan}_{x}(N)$ and $\operatorname{Swan}_{t}\left(\pi_{*} \overline{\mathbb{Q}}_{l}\right)$ vanish.

Once we know that $\pi$ is tame and that $N$ is tame, we argue as follows. Pick a point $s$ in $S$. Let the points in $X$ lying over $s$ be the $x_{i}$, with local degree $d_{i}$ over $s$. Then the $I(s)$ representation $M(s)$ is the direct sum of the $d_{i}$-fold Kummer inductions $\left[d_{i}\right]_{*} N\left(x_{i}\right)$ (compare [Ka1], proof of 3.5.2)

$$
M(s) \approx \bigoplus_{i}\left[d_{i}\right]_{*} N\left(x_{i}\right) .
$$

Since $M(s)$ is a tame pseudoreflection but not a reflection, all but one of the terms $\left[d_{i}\right]_{*} N\left(x_{i}\right)$ must be the trivial representation, and the remaining one, say $\left[d_{1}\right]_{*} N\left(x_{1}\right)$, must itself be a tame pseudoreflection but not a reflection.

If $\left[d_{i}\right]_{*} N\left(x_{i}\right)$ is trivial, then $N\left(x_{i}\right)$ must be trivial (since it is a direct factor of $\left.\left[d_{i}\right]^{*}\left[d_{i}\right]_{*} N\left(x_{i}\right)\right)$, whence $d_{i}=1$.

If $\left[d_{1}\right]_{*} N\left(x_{1}\right)$ is a tame pseudoreflection but not a reflection, then by the same direct factor argument $N\left(x_{1}\right)$ is either trivial or is itself a tame pseudoreflection. If $N\left(x_{1}\right)$ is trivial and $\left[d_{1}\right]_{*} N\left(x_{1}\right)$ is a tame pseudoreflection, then $N\left(x_{1}\right)$ has rank one and $d_{1}=2$; but then $\left[d_{1}\right]_{*} N\left(x_{1}\right)$ is a reflection, and so this case does not occur.

If $N\left(x_{1}\right)$ and $\left[d_{1}\right]_{*} N\left(x_{1}\right)$ are both tame pseudoreflections, we claim $d_{1}=1$. If $N\left(x_{1}\right)$ is a unipotent pseudoreflection, then $\operatorname{rank}\left(N\left(x_{1}\right)\right) \geq 2$ and the semisimplification of $\left[d_{1}\right]_{*} N\left(x_{1}\right)$ is $\operatorname{rank}\left(N\left(x_{1}\right)\right)$ copies of $\left[d_{1}\right]_{*} \overline{\mathbb{Q}}_{l}$, which contains at least two nontrivial characters (counting multiplicity) if $d_{1} \geq 2$. Finally, if $N\left(x_{1}\right)$ is a nonunipotent pseudoreflection, say, of determinant $\rho \neq 1$, 
then $N\left(x_{1}\right)$ is semisimple and $\left[d_{1}\right]_{*} N\left(x_{1}\right)$ contains all the $d_{1}$ th roots of $\rho$, each of which is a fortiori nontrivial. So in this case we must have $d_{1}=1$ if $\left[d_{1}\right]_{*} N\left(x_{1}\right)$ is to be a pseudoreflection.

Therefore each $d_{i}$ is 1 , which is to say that $\pi: X \rightarrow \mathbb{P}^{1}$ is étale over $\mathbb{A}^{1}$, as required. Q.E.D.

5.9. Lemma. Over an algebraically closed field $k$, let $S$ be a finite nonempty subset of $\mathbb{A}^{1}(k), l$ a prime number invertible in $k$, and $M$ a lisse $\overline{\mathbb{Q}}_{l}$-sheaf on $\mathbb{A}^{1}-S$. Suppose that

(1) $M$ is irreducible;

(2) $M$ is tame at $\infty$;

(3) at each point $s$ in $S$, the local monodromy of $M$ is a tame pseudoreflection $\gamma_{s}$, which is not a reflection and whose determinant $\operatorname{det}\left(\gamma_{s}\right)$ has finite order $n_{s}$ (as a character of the inertia group $I(s)$ ).

Then

(1) $\operatorname{det}(M)$ has finite order, equal to the l.c.m. of the integers $n_{s}$,

(2) either $M$ is Lie-irreducible or $M$ has finite monodromy.

Proof. Since $M$ is tame on $\mathbb{A}^{1}-S, \operatorname{det}(M)$ is tame on $\mathbb{A}^{1}-S$, so its monodromy group is the subgroup of $\mathrm{GL}(1)$ generated by the local monodromies at all the points $s$ in $S$. This proves (1).

By [Ka3, Proposition 1], our irreducible $M$ is either Lie-irreducible, or induced, or is the tensor product $N \otimes L$ of a Lie-irreducible $L$ of lower rank with an $N$ having finite monodromy. Suppose $M$ is not Lie-irreducible. We have already eliminated the induced case. Therefore $M$ is the tensor product $N \otimes L$ of a Lie-irreducible $L$ of lower rank with an $N$ having finite monodromy. Since a pseudoreflection is not nontrivially a tensor product (compare [Kal, proof of 3.5.7]), $L$ must have rank one, and as $\operatorname{det}(M)$ is of finite order, it follows that $L$ itself is of finite order, whence $N \otimes L$ has finite monodromy. Q.E.D.

5.10. (Mitchell [Mi]) Theorem. Over an algebraically closed field $k$, let $S$ be a finite nonempty subset of $\mathbb{A}^{1}(k), l$ a prime number invertible in $k$, and $M$ a lisse $\overline{\mathbb{Q}}_{1}$-sheaf on $\mathbb{A}^{1}-S$. Suppose that

(1) $M$ is irreducible;

(2) $M$ is tame at $\infty$;

(3) at each point $s$ in $S$, the local monodromy of $M$ is a tame pseudoreflection $\gamma_{s}$, which is not a reflection and whose determinant $\operatorname{det}\left(\gamma_{s}\right)$ has finite order $n_{s}$ (as a character of the inertia group $I(s)$ );

(4) $M$ has finite monodromy.

Then

(1) $\operatorname{rank}(M) \leq 4$,

(2) if $\operatorname{rank}(M)=3$ or 4 then every $n_{s}=3$.

Proof. Let us denote $n:=\operatorname{rank}(M)$. Since $M$ is not induced, $G_{\text {geom }}$ is a finite irreducible subgroup of $\mathrm{GL}(n)$, which is "primitive" (i.e., the given representation is not induced) and which is generated by pseudoreflections. According to Mitchell [Mi, Theorem 1], if $n>4$ then the only pseudoreflections in such 
a group are reflections, so each $n_{s}=2$ (this case does not occur for us). If $n$ is 3 or 4, then classification (cf. [Mi]) shows that any pseudoreflection in such a group has order 2 or 3 . So each $n_{s}$ is 2 or 3 , and $n_{s} \neq 2$ by hypothesis. Q.E.D.

5.11. Theorem. Over an algebraically closed field $k$, let $S$ be a finite nonempty subset of $\mathbb{A}^{1}(k), l$ a prime number invertible in $k$, and $M$ a lisse $\overline{\mathbb{Q}}_{l}$-sheaf on $\mathbb{A}^{1}-S$. Suppose that

(1) $M$ is irreducible;

(2) $M$ is tame at $\infty$;

(3) At each point $s$ in $S$, the local monodromy of $M$ is a tame pseudoreflection $\gamma_{s}$, which is not a reflection and whose determinant $\operatorname{det}\left(\gamma_{s}\right)$ has finite order $n_{s}$ (as a character of the inertia group $I(s)$ ).

Suppose that either

(a) $n:=\operatorname{rank}(M)>4$, or

(b) $n:=\operatorname{rank}(M)>2$ and some $n_{s} \neq 3$.

Then

(1) $M$ is Lie-irreducible;

(2) $\left(G_{\text {geom }}\right)^{0}=\mathrm{SL}(n)$ if some $n_{s} \neq 1$ or if $n$ is odd;

(3) if all $n_{s}=1$ and $n$ is even, then $\left(G_{\text {geom }}\right)^{0}$ is either $\operatorname{SL}(n)$ or $\operatorname{Sp}(n)$;

(4) If $\left(G_{\text {geom }}\right)^{0}=\operatorname{SL}(n)$, put $N:=$ l.c.m. of the $n_{s}:=\operatorname{order}$ of $\operatorname{det}(M)$.

Then $G_{\text {geom }}$ is $\left\{A\right.$ in $\left.\mathrm{GL}(n) \mid \operatorname{det}(A)^{N}=1\right\}$.

Proof. That $M$ is Lie-irreducible follows from the previous two results. Since $\operatorname{det}(M)$ is of finite order, $\left(G_{\text {geom }}\right)^{0}$ is semisimple (being an irreducible connected subgroup of $\operatorname{SL}(n))$. Conclusions (2) and (3) then follow from the Pseudoreflection Theorem [Ka1, 1.5] applied to $\operatorname{Lie}\left(G_{\text {geom }}\right)$ in its given representation and to any of the pseudoreflections $\gamma_{s}$. Conclusion (4) is trivial, since $\operatorname{det}(M)$ has order $N$. Q.E.D.

5.12. Application to weakly supermorse functions. Let $k$ be finite field, $f(x)$ in $k[x]$ weakly supermorse of degree $d$, and $\chi$ a character of $k^{\times}$with $\chi^{d} \neq \mathbb{1}$. In Key Lemma 5.7, we have proven:

(1) the object $\left(\mathscr{F} *_{!+} j \mathscr{L}_{\chi}\right)[1]$ is a geometrically irreducible Fourier sheaf, say $M$, of generic rank $d-1$;

(2) the trace function of $M$ is $t \mapsto-\sum_{x} \chi(t-f(x))$;

(3) the geometrically irreducible Fourier sheaf $\operatorname{NFT}_{\psi}(M)$ is geometrically isomorphic to $\mathscr{G} \otimes\left(j_{!} \mathscr{L}_{\bar{\chi}}\right)$;

(4) $M$ is tame at $\infty$;

(5) $M$ is lisse on $\mathbb{A}^{1}$ outside the $d-1$ critical values of $f$, i.e., outside the points $f(\alpha)$ for $\alpha$ a zero of $f^{\prime}(x)$. At each critical value $f(\alpha)$, the local monodromy of $M$ is a tame pseudoreflection whose determinant, as character of $I(f(\alpha))$, is $\mathscr{L}\left(\chi \chi_{2}\right)(x-f(\alpha))$.

Denote by $S \subset \mathbb{A}^{1}$ the set of critical values of $f$. Denote by $N$ the exact order of the character $\chi \chi_{2}$. We may apply Theorem 5.11 to $\mathbb{A}^{1}-S$ and the 
sheaf $M$. All the finite local monodromies $\gamma_{s}$ are pseudoreflections, whose determinants have the same finite order $N$. So we find

5.13. Theorem. Notations are as in 5.12 above. Let $k$ be a finite field of characteristic $p \neq 2, f(x)$ in $k[x]$ weakly supermorse of degree $d$, and $\chi$ a character of $k^{\times}$with $\chi^{d} \neq \mathbb{1}$. Denote by $N$ the order of the character $\chi \chi_{2}$. Let $l$ be a prime number invertible in $k$. Then $G_{\text {geom }}$ for the lisse $\overline{\mathbb{Q}}_{l}$-sheaf $M$ on $\mathbb{A}^{1}-S$ of rank $d-1$, whose trace function is

$$
t \mapsto-\sum_{x} \chi(t-f(x))
$$

is given as follows.

(1) If $\chi=\chi_{2}$ is the quadratic character, then $d$ is odd and $G_{\mathrm{geom}}$ is $\operatorname{Sp}(d-1)$.

(2) If $\chi \neq \chi_{2}$, suppose the either

(a) $d>5$, or

(b) $d>3$ and $\left(\chi \chi_{2}\right)^{3} \neq \mathbb{1}$.

Then $\left(G_{\text {geom }}\right)^{0}=\operatorname{SL}(d-1)$ and $G_{\text {geom }}$ is $\left\{A\right.$ in $\left.\operatorname{GL}(d-1) \mid \operatorname{det}(A)^{N}=1\right\}$.

Proof. Parts (2a) and (2b) are immediate from Theorem 5.11. For part (1), we must explain why if $\chi=\chi_{2}$ then $d$ is odd and why we have an a priori inclusion $G_{\text {geom }} \subset \operatorname{Sp}(d-1)$. The nontriviality of $\left(\chi_{2}\right)^{d}$ implies that $d$ is odd. Over $\mathbb{A}^{1}-S$, consider the proper smooth family of hyperelliptic curves $\varphi: \mathscr{C} \rightarrow \mathbb{A}^{1}-S$ whose fibre over $t$ is the complete nonsingular model of the affine curve of equation

$$
\mathscr{C}_{t}: y^{2}=t-f(x)
$$

Because $d$ is odd, $\mathscr{C}$ is the disjoint union of this affine curve and of a single section " $\infty$ ". The sheaf $M$ is none other than $R^{1} \varphi_{1} \overline{\mathbb{Q}}_{l}$. [To see this, we argue as follows. Both are lisse sheaves on $\mathbb{A}^{1}-S$, and both have the same trace function, namely,

$$
t \mapsto-\sum_{x} \chi_{2}(t-f(x))
$$

So by Chebataroff, both have isomorphic semisimplifications as lisse sheaves on $\mathbb{A}^{1}-S$; but $M$ is irreducible (because it is geometrically irreducible), and hence $R^{1} \varphi_{1} \overline{\mathbb{Q}}_{l}$ must itself be irreducible (since its semisimplification $\sim M$ is irreducible); therefore, there exists an isomorphism $M \approx R^{1} \varphi_{!} \overline{\mathbb{Q}}_{l}$ as lisse sheaves on $\mathbb{A}^{1}-S$.] This description of $M$ shows the existence of a symplectic autoduality on $M$, corresponding to the cup-product pairing on $R^{1} \varphi_{!} \overline{\mathbb{Q}}_{l}(1 / 2)$. Therefore we have an a priori inclusion $G_{\text {geom }} \subset \operatorname{Sp}(d-1)$ if $\chi=\chi_{2}$. Q.E.D.

\subsection{Return to the proof of Theorem 5.4.}

Proof. First of all, if $\chi=\chi_{2}, d$ is odd and $G_{\text {geom }} \subset \operatorname{Sp}(d-1)$, by the same argument as used in the proof of 5.13. Moreover, if $\chi \neq \chi_{2}$. Determinant Lemma 5.2 shows that every element $A$ in $G_{\text {geom }}$ has $\operatorname{det}(A)^{N}=1$. So in 
order to prove Theorem 5.4, it suffices to show that in case (1) (resp. case (2)) $G_{\text {geom }}$ contains $\operatorname{Sp}(d-1)$ (resp. $\left\{A\right.$ in $\left.\operatorname{GL}(d-1) \mid \operatorname{det}(A)^{N}=1\right\}$ ).

Now $G_{\text {geom }}$ for $\mathscr{H}^{-2}(M) \mid U$ contains the $G_{\text {geom }}$ for the pullback of $\mathscr{H}^{-2}(M) \mid U$ to any geometrically connected $X$ by any map $X \rightarrow U \otimes \bar{k}$. We will show that for all but finitely many values of " $a$ " in $\bar{k}$, the polynomial $g_{a}(x):=-f(x)-a x$ is weakly supermorse. For such a good " $a_{0}$ ", we will take $X:=\left\{(a, b)\right.$ in $U$ with $\left.a=a_{0}\right\}$ (so $X$ is the complement in the $\mathbb{A}^{1}$ of $b$ 's of the critical values of $g_{a}$ ) and apply the previous result to the weakly supermorse function $g_{a}$, whose sheaf " $M$ " is precisely the pullback of $X$ of $\mathscr{H}^{-2}(M) \mid U$. Since this pullback already has a big enough $G_{\text {geom }}$, we are done. Q.E.D.

It remains only to prove the following lemma.

5.15. Lemma. Let $k$ be a field, $d \geq 2$ an integer such that $d$ is invertible in $k$, and $f(x)$ in $k[x]$ a polynomial in one variable over $k$ of degree $d$ whose second derivative $f^{\prime \prime}(x)$ is not identically zero. Then there exists a nonzero polynomial $R(t)$ in $k[t]$ such that for any overfield $K$ of $k$ and any element $a$ in $K$ with $R(a) \neq 0$, the polynomial $f_{a}(x):=f(x)+a x$ in $K[x]$ is weakly supermorse.

Proof. The derivative $f_{a}^{\prime}$ of $f_{a}$ is the polynomial $f^{\prime}(x)+a$, which has degree $d-1$. Its discriminant, given by some universal formula, is a polynomial $\Delta(a)$ in $k[a]$. We first show that the polynomial $\Delta$ is nonzero, i.e., that $f_{a}^{\prime}$ has all distinct roots for general values of " $a$ ". Since $f^{\prime \prime}(x)$ is nonzero, $f^{\prime \prime}$ has only finitely many zeros, say $\gamma_{i}$ 's, in $\bar{k}$. Since $f^{\prime \prime}$ is the derivative of $f_{a}^{\prime}, f_{a}^{\prime}$ has all its zeros simple provided that none of the $\gamma_{i}$ 's is a zero of $f_{a}^{\prime}$, i.e., provided that $a \neq-f^{\prime}\left(\gamma_{i}\right)$ for each root $\gamma_{i}$ of $f^{\prime \prime}$. Thus $\Delta$ is nonzero.

For any quantity $a$ with $\Delta(a) \neq 0$, the polynomial $f_{a}$ is weakly supermorse if and only if $f_{a}$ separates the zeros of $f^{\prime}(x)+a$. Consider the product, over all pairs $(\alpha, \beta)$ of distinct roots of $f_{a}^{\prime}=f^{\prime}(x)+a$, of the difference $f_{a}(\alpha)-f_{a}(\beta)$, say

$$
D(a):=\prod_{(\alpha, \beta) \text { dist. roots of } f_{a}^{\prime}}\left(f_{a}(\alpha)-f_{a}(\beta)\right) .
$$

This, too, is given by some universal formula: it is an element of the ring $k[a, 1 / \Delta(a)]$, say $D(a)=P(a) /($ a power of $\Delta(a))$. We must show that it is nonzero. For in this case, the product $P(t) \Delta(t)$ will function as the required $R(t)$.

To understand $D(a)$ as a function of $a$, we work over the ring $k[a][1 / \Delta(a)]$ and pass to the finite étale $\mathscr{S}_{d-1}$-torsor over it, which represents the functor “ $d-1$ distinct roots of $f^{\prime}(x)+a$ ". Concretely, if we write $f(x)=c x^{d}+$ lower terms, this is the ring

$$
\mathscr{R}:=k[a][1 / \Delta(a)]\left[z_{1}, \ldots, z_{d-1}\right] / \mathscr{F}
$$

where $\mathscr{F}$ is the ideal defined by the equation

$$
\prod_{i \neq j}\left(z_{i}-z_{j}\right)=\Delta(a)
$$


and by the $d$ relations obtained by equating like powers of $x$ in

$$
c d \prod_{i}\left(x-z_{i}\right)=f^{\prime}(x)+a .
$$

Then $D(a)$ makes sense as the element of $\mathscr{R}$ given by

$$
\prod_{i \neq j}\left(f_{a}\left(z_{i}\right)-f_{a}\left(z_{j}\right)\right)
$$

Because this element is visibly invariant under the permutation action of $\mathscr{S}_{d-1}$ on the $z_{i}$, it in fact lies in the ground-ring $k[a][1 / \Delta(a)]$, justifying the $D(a)$ notation.

To show that this element is nonzero, we argue by contradiction. If $D(a)$ is identically zero, then viewed as an element of $\mathscr{R}$ it vanishes identically on each connected component of $\operatorname{Spec}(\mathscr{R})$. But $\mathscr{R}$ is finite étale over the normal ring $k[a][1 / \Delta(a)]$ and so is itself normal, and hence each connected component, say $\operatorname{Spec}\left(\mathscr{R}_{\nu}\right)$, has $\mathscr{R}_{\nu}$ an integral domain, which is finite étale over $k[a][1 / \Delta(a)]$. Because $\mathscr{R}_{\nu}$ is an integral domain in which $D(a)$ vanishes, there exists a pair of indices $i \neq j$ such that

$$
f_{a}\left(z_{i}\right)-f_{a}\left(z_{j}\right)=0 \text { in } \mathscr{R}_{\nu} .
$$

Because $\mathscr{R}_{\nu}$ is étale over $k[a][1 / \Delta(a)]$, the derivation $d / d a$ of $k[a][1 / \Delta(a)]$ extends uniquely to a $k$-linear derivation, say $\delta$, of $\mathscr{R}_{\nu}$, satisfying $\delta(a)=1$. We compute

$$
\delta\left(f_{a}\left(z_{i}\right)\right)=\delta\left(f\left(z_{i}\right)+a z_{i}\right)=f^{\prime}\left(z_{i}\right) \delta\left(z_{i}\right)+a \delta\left(z_{i}\right)+z_{i}=z_{i},
$$

the last equality because $f^{\prime}\left(z_{i}\right)+a=0$. Similarly, $\delta\left(f_{a}\left(z_{j}\right)\right)=z_{j}$. So applying $\delta$ to the equality

$$
f_{a}\left(z_{i}\right)-f_{a}\left(z_{j}\right)=0 \text { in } \mathscr{R}_{\nu},
$$

we find that $z_{i}-z_{j}=0$ in $\mathscr{R}_{\nu}$, a contradiction since $z_{i}-z_{j}$ is invertible in $\mathscr{R}$ and, hence, in $\mathscr{R}_{\nu}$. Q.E.D.

5.16. Remark. What becomes of Theorem 5.4 if we allow for $\chi$ a nontrivial character with $\chi^{d}=\mathbb{1}$ ? First of all, if $\chi$ is nontrivial but $\chi^{d}$ is trivial, then $\mathscr{H}^{-2}(M) \mid U$ has a lisse rank 1 subsheaf, which is pure of weight 0 and geometrically constant. The quotient of $\mathscr{H}^{-2}(M) \mid U$ by this lisse rank 1 subsheaf, say $\mathscr{F}$, is lisse of rank $d-2$ and pure of weight 1 . By the Irreducibility Theorem 2.1.1 (and Corollary 2.1.4) this quotient $\mathscr{F}$ is geometrically irreducible.

To see this structure when $\chi^{d}$ is trivial, suppose to fix ideas that $f$ is monic. Write $d=e p^{\nu}$ with $e$ prime to $p$. Since $\chi$ has order prime to $p, \chi^{d}=1 \Rightarrow$ $\chi^{e}=\mathbb{1}$; but any monic $f(x)$ of degree $d$ is an $e$ th power in $k((1 / x))$, so $\mathscr{L}_{\chi(f(x))} \mid \operatorname{Spec}(k((1 / x)))$ is trivial. So for $(a, b)$ in $U$, we have a short exact sequence of sheaves on $\mathbb{P}^{1}$

$$
0 \rightarrow j_{!} \mathscr{L}_{\chi\left(f_{a, b}\right)} \rightarrow j_{*} \mathscr{L}_{\chi\left(f_{a, b}\right)} \rightarrow\left(\overline{\mathbb{Q}}_{l}\right)_{\infty} \rightarrow 0 .
$$

The long exact cohomology sequence on $\mathbb{P}^{1}$ gives, for $(a, b)$ in $U$,

$$
0 \rightarrow \overline{\mathbb{Q}}_{l} \rightarrow \mathscr{H}^{-2}(M)_{a, b} \rightarrow H_{c}^{1}\left(\mathbb{P}^{1} \otimes \bar{k}, j_{*} \mathscr{L}_{\chi\left(f_{a, b}\right)}\right) \rightarrow 0,
$$


and the last term is pure of weight 1 by [De, 3.2.3].

Concretely, then, for $f$ monic of degree $d$, the trace function of $\mathscr{F}$ is given by the following recipe: For $E$ a finite extension of $k, \chi_{E}$ the character $\chi \circ \operatorname{Norm}_{E / k}$ of $E^{\times}$, and $(a, b)$ in $U(E)$, we have

$$
\operatorname{Trace}\left(F_{(a, b), E} \mid \mathscr{F}\right)=-1-\sum_{x \text { in } E} \chi_{E}(f(x)+a x+b) .
$$

If we drop the requirement that $f$ be monic and write

$$
f(x)=c x^{d}+\text { lower terms }
$$

then the trace function is

$$
\operatorname{Trace}\left(F_{(a, b), E} \mid \mathscr{F}\right)=-\chi_{E}(c)-\sum_{x \text { in } E} \chi_{E}(f(x)+a x+b) .
$$

5.17. Theorem. Notation is as in 5.1. Suppose that $d \geq 3$ is prime to $p$, that $f^{\prime \prime}(x)$ is not identically zero (whence char $(k) \neq 2$ ), that $\chi$ is nontrivial, and that $\chi^{d}=\mathbb{1}$. Denote by $G_{\text {geom }}$ the geometric monodromy group of the (geometrically irreducible) lisse sheaf $\mathscr{F}$ (defined above) of rank $d-2$. Denote by $\chi_{2}$ the unique character of order two of $k^{\times}$, and denote by $N$ the (exact) order of the character $\chi \chi_{2}$ of $k^{\times}$. Then:

(1) If $\chi=\chi_{2}$ is the quadratic character, then $d$ is even and $G_{\text {geom }}$ is $\mathrm{Sp}(d-2)$.

(2) If $\chi \neq \chi_{2}$, suppose that either

(a) $d>6$, or

(b) $d>4$ and $\left(\chi \chi_{2}\right)^{3} \neq \mathbb{1}$.

Then $\left(G_{\text {geom }}\right)^{0}=\mathrm{SL}(d-2)$, and $G_{\text {geom }}=\left\{A\right.$ in $\left.\mathrm{GL}(d-2) \mid \operatorname{det}(A)^{N}=1\right\}$.

Proof. The idea is the same, to prove the theorem already for the restriction of $\mathscr{F}$ to the line in $U$ where " $a$ " is frozen to a value for which $f(x)+a x$ is weakly supermorse.

For an $f$, which is weakly supermorse of degree $d$, and a nontrivial $\chi$ of order dividing $d$, the sheaf $M$ has a rank 1 geometrically constant subsheaf of weight zero and the quotient, say $\bar{M}$, is a lisse sheaf of rank $d-2$, which is pure of weight 1 . The extension by direct image to $\mathbb{A}^{1}$ of $\bar{M}$ is the Fourier Transform not of $\mathscr{G} \otimes j_{!} \mathscr{L}_{\bar{\chi}}$ but rather of its middle extension $j_{*} j^{*}\left(\mathscr{G} \otimes j_{!} \mathscr{L}_{\chi}\right)$. In this way, we prove the following variant of Key Lemma 5.7.

5.18. Variant Key Lemma. If $f=c x^{d}+\cdots$ is weakly supermorse of degree $d$, if $\chi$ is nontrivial, and if $\chi^{d}=1$, then:

(1) the highest weight quotient of the object $\left(\mathscr{F}_{*_{!+}} j_{!} \mathscr{L}_{\chi}\right)[1]$ is a geometrically irreducible Fourier sheaf, say $\bar{M}$, of generic rank $d-2$;

(2) the trace function of $\bar{M}$ is $t \mapsto-\chi(c)-\sum_{x} \chi(t-f(x))$;

(3) the geometrically irreducible Fourier sheaf $\operatorname{NFT}_{\psi}(\bar{M})$ is geometrically isomorphic to $j_{*} j^{*}\left(\mathscr{G} \otimes\left(j_{1} \mathscr{L}_{\bar{\chi}}\right)\right)$;

(4) $\bar{M}$ is tame at $\infty$; 
(5) $\bar{M}$ is lisse on $\mathbb{A}^{1}$ outside the $d-1$ critical values of $f$, i.e., outside the points $f(\alpha)$ for $\alpha$ a zero of $f^{\prime}(x)$. At each critical value $f(\alpha)$, the local monodromy of $M$ is a tame pseudoreflection whose determinant, as character of $I(f(\alpha))$, is $\mathscr{L}_{\left(\chi \chi_{2}\right)(x-f(\alpha))}$.

From this point on, the proof is exactly the same. Q.E.D.

\section{APPENDIX (D'APRES DELIGNE)}

In this appendix, we work over an algebraically closed field $k$ and fix a prime $l \neq \operatorname{char}(k)$. We fix an integer $n \geq 1$, and denote by $\mathbb{P}$ the $n$-dimensional projective space $\mathbb{P}^{n}$ over $k$. We denote by $\mathscr{P}$ (sic) the dual projective space over $k$, i.e., $\mathscr{P}$ is the space of hyperplanes in $\mathbb{P}$. We denote by $\mathscr{H} \subset \mathbb{P} \times \mathscr{P}$ the universal hyperplane: in terms of projective coordinates $\left(X_{0}, \ldots, X_{n}\right)$ for $\mathbb{P}$ and $\left(Y_{0}, \ldots, Y_{n}\right)$ for $\mathscr{P}, \mathscr{H}$ is defined by the equation $\sum Y_{i} X_{i}=0$.

A.1. Lemma. Consider the inclusion $\beta: \mathbb{P} \times \mathscr{P}-\mathscr{H} \rightarrow \mathbb{P} \times \mathscr{P}$ as a morphism of $\mathbb{P}$-schemes. Zariski locally on $\mathbb{P}$, this morphism $\beta$ is $\mathbb{P}$-isomorphic to the product of $\mathbb{P}$ with the inclusion $j: \mathscr{A} \rightarrow \mathscr{P}$ of the affine open set $\mathscr{A}$ in $\mathscr{P}$ where $Y_{0}$ is invertible.

Proof. For each integer $0 \leq i \leq n$, denote by $U_{i} \subset \mathbb{P}$ the affine open set where $X_{i}$ is invertible and denote by $\mathscr{U}_{i} \subset \mathscr{P}$ the affine open set where $Y_{i}$ is invertible. We claim that over $U_{i}$, the morphism in question is $U_{i}$-isomorphic to $U_{i} \times\left(\mathscr{U}_{i} \rightarrow \mathscr{P}\right)$. This will prove the lemma, since by renumbering the coordinates in $\mathscr{P}, \mathscr{U}_{i} \rightarrow \mathscr{P}$ becomes isomorphic to $\mathscr{U}_{0} \rightarrow \mathscr{P}$. To prove the claim, it suffices, again by renumbering the coordinates, to treat the case $i=0$. In this case, $U_{0}$ is the $\mathbb{A}^{n}$ with coordinates to $x_{i}:=X_{i} / X_{0}$ for $i=1, \ldots, n$, and inside $U_{0} \times \mathscr{P}, \mathscr{H}$ is defined by the vanishing of $Y_{0}+\sum_{i \geq 1} x_{i} Y_{i}$. After the $U_{0}$-automorphism of $U_{0} \times \mathbb{P}$ defined by

$$
\left(x_{i} \text { 's }, Y_{0}, Y_{1}, \ldots, Y_{n}\right) \mapsto\left(x_{i} \text { 's }, Y_{0}+\sum_{i \geq 1} x_{i} Y_{i}, Y_{1}, \ldots, Y_{n}\right),
$$

$\mathscr{H}$ is defined inside $U_{0} \times \mathscr{P}$ by the vanishing of $Y_{0}$. Q.E.D.

A.2. Lemma. Let $f: X \rightarrow \mathbb{P}$ be a separated morphism of finite type and $K$ an object of $D_{c}^{b}\left(X, \overline{\mathbb{Q}}_{1}\right)$. On the product $X \times \mathscr{P}$, consider the object $p r_{1}^{*} K$ and the Cartesian diagram

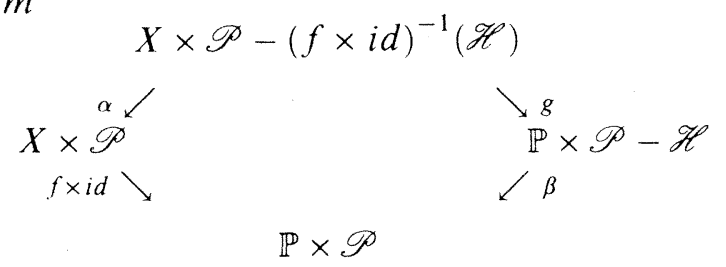

On $\mathbb{P} \times \mathscr{P}$, the natural map of adjunction

$$
\beta_{!} R g_{*}\left(\alpha^{*} p r_{1}^{*} K\right) \rightarrow R(f \times i d)_{*} \alpha_{!}\left(\alpha^{*} p r_{1}^{*} K\right)
$$

is an isomorphism. 
Proof. The question is Zariski local on $\mathbb{P} \times \mathscr{P}$, so in particular it is Zariski local on $\mathbb{P}$. By the previous lemma, Zariski locally on $\mathbb{P}$ the situation is isomorphic to the product of the two situations

$$
\begin{array}{rr}
K \text { on } X & \overline{\mathbb{Q}}_{l} \text { on } \mathscr{A} \\
f \downarrow & j \downarrow \\
\mathbb{P} & \mathscr{P}
\end{array}
$$

i.e., Zariski locally on $\mathbb{P}$ our Cartesian diagram is

$$
\begin{array}{lll} 
& X \times \mathscr{A} & \\
i d \times j \swarrow & & \searrow f \times i d \\
X \times \mathscr{P} & & \mathbb{P} \times \mathscr{A} \\
f \times i d \searrow & & \swarrow i d \times j \\
& \mathbb{P} \times \mathscr{P} &
\end{array}
$$

and the object $\alpha^{*} p r_{1}^{*} K$ on $X \times \mathscr{P}-(f \times i d)^{-1}(\mathscr{H})$ becomes the external tensor product $K \otimes \overline{\mathbb{Q}}_{l, \mathscr{A}}$ on $X \times \mathscr{A}$. By the Kunneth formulas for both ordinary and! direct images ([SGA4, XVII, 5.4.3] for! direct images, combined with biduality, [SGA $4 \frac{1}{2}$, Théorèmes de Finitude, 4.3] to deduce $*$ direct images) we have

$$
\begin{aligned}
\beta_{!} R g_{*}\left(\alpha^{*} p r_{1}^{*} K\right) & :=R \beta_{!} R g_{*}\left(\alpha^{*} p r_{1}^{*} K\right) \\
& =R(i d \times j)_{!} R(f \times i d)_{*}\left(K \otimes \overline{\mathbb{Q}}_{l, \mathscr{A}}\right) \\
& =R(i d \times j)_{!}\left(\left(R f_{*} K\right) \otimes \overline{\mathbb{Q}}_{l, \mathscr{A}}\right) \\
& =\left(R f_{*} K\right) \otimes\left(R j_{!} \overline{\mathbb{Q}}_{l, \mathscr{A}}\right)
\end{aligned}
$$

and, similarly,

$$
\begin{aligned}
R(f \times i d)_{*} \alpha_{!}\left(\alpha^{*} p r_{1}^{*} K\right): & =R(f \times i d)_{*} R \alpha_{!}\left(\alpha^{*} p r_{1}^{*} K\right) \\
& =R(f \times i d)_{*} R(i d \times j)_{!}\left(K \otimes \overline{\mathbb{Q}}_{l, \mathscr{A}}\right) \\
& =R(f \times i d)_{*}\left(K \otimes\left(R j ! \overline{\mathbb{Q}}_{l, \mathscr{A}}\right)\right) \\
& =\left(R f_{*} K\right) \otimes\left(R j \overline{\mathbb{Q}}_{l, \mathscr{A}}\right) . \quad \text { Q.E.D. }
\end{aligned}
$$

A.3. Lemma. Let $f: X \rightarrow \mathbb{P}$ be a separated morphism of finite type and $K$ an object of $D_{c}^{b}\left(X, \overline{\mathbb{Q}}_{l}\right)$. For $H \subset \mathbb{P}$ a hyperplane, denote by $\beta_{H}: \mathbb{P}-H \rightarrow \mathbb{P}$ the inclusion, and consider the Cartesian diagram

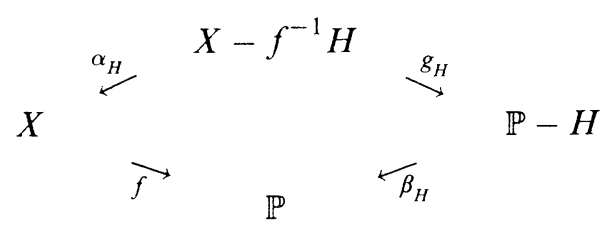

There exists a dense open set $\mathscr{U}$ in the space $\mathscr{P}$ of all hyperplanes in $\mathbb{P}$ such that if $H$ lies in $\mathscr{U}$ then the natural morphism of adjunction

$$
\left(\beta_{H}\right)_{!} R\left(g_{H}\right)_{*}\left(\alpha_{H}\right)^{*} K \rightarrow R f_{*}\left(\alpha_{H}\right)_{!}\left(\alpha_{H}\right)^{*} K
$$

is an isomorphism on $\mathbb{P}$. 
Proof. In the universal situation considered in Lemma A.2, we have an isomorphism

$$
\beta_{!} R g_{*}\left(\alpha^{*} p r_{1}^{*} K\right) \stackrel{\sim}{\rightarrow} R(f \times i d)_{*} \alpha_{!}\left(\alpha^{*} p r_{1}^{*} K\right) .
$$

By [SGA $4 \frac{1}{2}$, Théorèms de Finitude, 1.9], applied with $S=\mathscr{P}$ to the two situations

$$
\begin{array}{cc}
\alpha^{*} p r_{1}^{*} K \text { on } X \times \mathbb{P}-(f \times i d)^{-1}(\mathscr{H}) & \alpha_{!}\left(\alpha^{*} p r_{1}^{*} K\right) \text { on } X \times \mathscr{P} \\
g \downarrow & f \times i d\rfloor \\
\mathbb{P} \times \mathscr{P}-\mathscr{H} & \mathbb{P} \times \mathscr{P},
\end{array}
$$

both viewed as morphisms of $\mathscr{P}$-schemes, together with the proper base change theorem to handle $\alpha_{1}$ and $\beta_{1}$, there exists a dense open set $\mathscr{U}$ in $\mathscr{P}$ over which formation of both sides of the "universal" isomorphism $(*)$ above commutes with passages to fibres over $\mathscr{P}$. Taking the fibre over a point in $\mathscr{U}$ corresponding to a hyperplane $H$ in $\mathbb{P}$ gives the required isomorphism

$$
\left(\beta_{H}\right)_{!} R\left(g_{H}\right)_{*}\left(\alpha_{H}\right)^{*} K \stackrel{\sim}{\rightarrow} R f_{*}\left(\alpha_{H}\right)_{!}\left(\alpha_{H}\right)^{*} K \text {. Q.E.D. }
$$

Applying the functor $R \Gamma(\mathbb{P}$,$) , we find$

A.4. Corollary. Hypotheses and notation are as in Lemma A3. There exists a dense open $\mathscr{U}$ in $\mathscr{P}$ such that if $H$ lies in $\mathscr{U}$, we have an isomorphism $R \Gamma_{c}\left(\mathbb{P}-H, R\left(g_{H}\right)_{*}\left(\alpha_{H}\right)^{*} K\right) \cong R \Gamma\left(X,\left(\alpha_{H}\right)_{!}\left(\alpha_{H}\right)^{*} K\right)$ and a consequent isomorphism of cohomology groups

$$
H_{C}^{i}\left(\mathbb{P}-H, R\left(g_{H}\right)_{*}\left(\alpha_{H}\right)^{*} K\right) \cong H^{i}\left(X,\left(\alpha_{H}\right)_{!}\left(\alpha_{H}\right)^{*} K\right)
$$

for every $i$.

A.5. Corollary. (Weak Lefschetz Theorem). Hypotheses and notation are as in Lemma A3. Suppose in addition that the morphism $f: X \rightarrow \mathbb{P}$ is quasi-finite and the object $K$ on $X$ is perverse. Then there exists a dense open $\mathscr{U}$ in $\mathscr{P}$ such that if $H$ lies in $\mathscr{U}$, the cohomology groups $H^{i}\left(X,\left(\alpha_{H}\right)_{!}\left(\alpha_{H}\right)^{*} K\right)$ vanish for $i<0$ and (equivalently) for which the restriction maps

$$
H^{i}(X, K) \rightarrow H^{i}\left(f^{-1} H, K \mid f^{-1} H\right)
$$

are isomorphisms for $i<-1$, and injective for $i=-1$.

Proof. If we denote by $i_{H}: f^{-1} H \rightarrow X$ the inclusion, the short exact sequence (more precisely, the triangle) on $X$

$$
0 \rightarrow\left(\alpha_{H}\right)_{!}\left(\alpha_{H}\right)^{*} K \rightarrow K \rightarrow\left(i_{H}\right)_{*}\left(i_{H}\right)^{*} K \rightarrow 0
$$

leads to a long exact cohomology sequence, which shows the asserted equivalence. To show that $H^{i}\left(X,\left(\alpha_{H}\right)_{!}\left(\alpha_{H}\right)^{*} K\right)=0$ for $i<0$, it suffices by Corollary A.4 to show that $H_{c}^{i}\left(\mathbb{P}-H, R\left(g_{H}\right)_{*}\left(\alpha_{H}\right)^{*} K\right)=0$ for $i<0$. By duality, this is equivalent to showing that

$$
H^{i}\left(\mathbb{P}-H, R\left(g_{H}\right)_{!}\left(\alpha_{H}\right)^{*} D K\right)=0 \text { for } i>0 .
$$


Because $f$ is quasi-finite so is $g_{H}$, and hence $R\left(g_{H}\right)_{!}=\left(g_{H}\right)_{\text {! }}$ preserves semiperversity. Since $K$ is perverse, $\left(\alpha_{H}\right)^{*} D K$ is perverse, and hence $R\left(g_{H}\right)_{!}\left(\alpha_{H}\right)^{*} D K$ is semiperverse. Because $\mathbb{P}-H$ is affine, the cohomology groups $H^{i}(\mathbb{P}-H$, semiperverse $)=0$ for $i>0$, by the cohomological dimension of affines (or, more fancily, by the fact that because the structural map $\pi: \mathbb{P}-H \rightarrow \operatorname{Spec}(k)$ is affine, $R \pi_{*}$ (semiperverse on $\mathbb{P}-H$ ) is semiperverse on $\operatorname{Spec}(k))$. Q.E.D.

In order to state the next corollary, we recall the notion of relative cohomology modulo a closed set. If $Z$ is closed in $X, U:=X-Z$ the open complement, $j: U \rightarrow X$ the open immersion, and $i: Z \rightarrow X$ the closed immersion, then for $K$ on $X$ one defines

$$
H^{i}(X \bmod Z ; K):=H^{i}\left(X, j, j^{*} K\right) \text {. }
$$

From the triangle

$$
0 \rightarrow j_{!} j^{*} K \rightarrow K \rightarrow i_{*} i^{*} K \rightarrow 0,
$$

one gets the long exact cohomology sequence

$$
\cdots \rightarrow H^{i}(X \bmod Z ; K) \rightarrow H^{i}(X, K) \rightarrow H^{i}\left(Z, i^{*} K\right) \rightarrow \cdots
$$

A.6. Corollary (Generic duality formula for relative cohomology). Hypotheses and notation are as in Lemma A.3. Suppose in addition that the map $f: X \rightarrow \mathbb{P}$ factors as the composite

$$
X \stackrel{j}{\longrightarrow} \bar{X} \stackrel{i}{\longrightarrow} \mathbb{P}
$$

with $j$ an open immersion and $i$ a closed immersion. In other words, $\bar{X}$ is a closed subscheme of $\mathbb{P}$ and $X$ is open in $\bar{X}$. We denote by $Y$ in $\bar{X}$ the closed complement of $X, X=\bar{X}-Y$. Then for any object $K$ of $D_{c}^{b}\left(X, \overline{\mathbb{Q}}_{l}\right)$, there exists a dense open set $\mathscr{U}$ in $\mathscr{P}$ of hyperplanes such that if $H$ lies in $\mathscr{U}$, the relative cohomology groups

$$
H^{i}(X \bmod X \cap H, K) \quad \text { and } \quad H^{-i}(\bar{X}-\bar{X} \cap H \bmod Y-Y \cap H, D K)
$$

are canonically dual for every integer $i$.

Proof. This is just the spelling out of Lemma A.4 in this case, together with duality. Q.E.D.

\section{REFERENCES}

[BBD] A. A. Beilinson, I. N. Bernstein and P. Deligne, Faisceaux pervers, entire contents of Analyse et Topologie sur les espaces singuliers. I, Conference de Luminy, Astérisque, vol. 100, Soc. Math. France, Paris, 1982.

[Br] J.-L. Brylinski, Transformations canoniques, dualité projective, théorie de Lefschetz, transformations de Fourier et sommes trigonométriques, Astérisque, vol. 140-141, Soc. Math. France, Paris, 1986, pp. 3-134.

[DL] H. Davenport and D. J. Lewis, Notes on congruences (I), Quarterly J. Math. Oxford (2) 14 (1963), 51-60; reprinted in The collected works of Harold Davenport, vol. 3, Academic Press, New York, 1977, pp. 1290-1299.

[De] P. Deligne, La conjecture de Weil. II, Inst. Hautes Études Sci. Publ. Math. 52 (1981), 313428. 
[Dw] B. Dwork, Generalized hypergeometric functions, Clarendon Press, Oxford, 1990.

[GKZ] I. M. Gelfand, M. M. Kapranov, and A. V. Zelevinsky, Generalized Euler integrals and A-hypergeometric functions, Adv. In Math. 84 (1990), 255-271.

[Ka1] N. Katz, Exponential sums and differential equations, Ann. of Math. Stud., vol. 124, Princeton Univ. Press, Princeton, NJ, 1990.

[Ka2] _ Monodromy of families of curves: applications of some results of Davenport-Lewis, Seminaire D.P.P. 1979/80, Birkhäuser, Basel-Boston, MA, 1981, pp. 171-195.

[Ka3] _ On the monodromy groups attached to certain families of exponential sums, Duke Math. J. 54 (1987), 41-56.

[Ka4] __ Perversity and exponential sums, Algebraic Number Theory-in honor of K. Iwasawa, Adv. Stud. Pure Math., vol. 17, Academic Press, New York, pp. 209-259.

[Ka5] _ Perversity and exponential sums. II: Estimates for and inequalities among A-numbers, Proceedings of the Barsotti Memorial Conference (Padua, 1991), to appear

[KaLa] N. Katz and G. Laumon, Transformation de Fourier et majoration de sommes exponentielles, Inst. Hautes Études Sci. Publ. Math. 62 (1986), 361-418.

[La] G. Laumon, Semicontinuité du conducteur de Swan (d'après P. Deligne), Caractéristique d'Euler-Poincaré, Seminaire E. N. S. 1978-79, Astérisque, vol. 82-83, Soc. Math. France, Paris, 1981, pp. 173-219.

[Mi] H. H. Mitchell, Determination of all primitive collineation groups in more than four variables which contain homologies, Amer. J. Math. 36 (1914), 1-12.

[SGA] A. Grothendieck, et al, Séminaire de Géométrie Algébrique du Bois-Marie, SGA 1, SGA 4 Parts I, II, and III, SGA $4 \frac{1}{2}$, SGA 5, SGA 7 Parts I and II, Lecture Notes in Math., vols. 224, 269-270-305, 569, 589, 288-340, Springer, New York, 1971 to 1977.

Department of Mathematics, Princeton University, Princeton, New Jersey 08544

E-mail address: nmk@ math.princeton.edu 